\title{
Fatigue Damage Assessment of Materials Under Uniaxial Variable Amplitude Loading Conditions
}

By

Mitesh Sharma

A thesis presented to the Ryerson University

in fulfillment of the

thesis requirement for the degree of

Master of Applied Science

in

Mechanical Engineering

pipnopnt?as

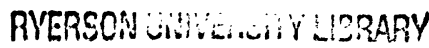

Toronto, Ontatio, Canada, 2003

(C) Mitesh Sharma 2003 
UMI Number: EC53451

\section{INFORMATION TO USERS}

The quality of this reproduction is dependent upon the quality of the copy submitted. Broken or indistinct print, colored or poor quality illustrations and photographs, print bleed-through, substandard margins, and improper alignment can adversely affect reproduction.

In the unlikely event that the author did not send a complete manuscript and there are missing pages, these will be noted. Also, if unauthorized copyright material had to be removed, a note will indicate the deletion.

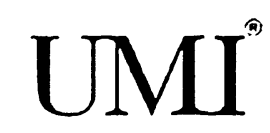

UMI Microform EC53451

Copyright 2009 by ProQuest LLC

All rights reserved. This microform edition is protected against unauthorized copying under Title 17, United States Code.

ProQuest LLC

789 East Eisenhower Parkway

P.O. Box 1346

Ann Arbor, MI 48106-1346 


\section{AUTHOR'S DECLARATION}

I hereby declare that I am the sole author of this thesis.

I authorize the Ryerson University to lend this thesis to other institutions or individuals for the purpose of scholarly research.

I further authorize the Ryerson University to reproduce this thesis by photocopying or by other means, in total or in part, at the request of other institutions or individuals for the purpose of scholarly research. 
The Ryerson University requires the signatures of all persons using or photocopying this thesis. Please sign below, and give address and date. 


\section{ABSTRACT}

This thesis intends to further apply an earlier developed energy based-critical fatigue damage parameter to assess the fatigue damage of different materials subjected to repeated random block histories. In fatigue damage assessment under variable loading conditions, further phenomenological factors of:

(i) sequence loading effect,

(ii) memory effect, and

(iii) the effect of small amplitude cycles below the material endurance limit have been introduced.

The effect due to sequence loading is studied for variable amplitude loading conditions. It is found that the loading sequence has a great influence on the cyclic stress-strain hysteresis loops and therefore on fatigue damage of materials. Memory effect concept has been carefully monitored and programmed to correspond to the closed hysteresis loops in each block loading history. The small cycles exceeding $50 \%$ of the fatigue endurance limit contributed to the accumulated damage.

A comparison of the predicted fatigue life results based on energy based-critical parameter including the phenomenological factors with the experimental live data reported in the literature has shown a good agreement. 


\section{ACKNOWLEDGEMENTS}

I wish to acknowledge and thank my supervisor Professor A. Varvani-Farahani for his strong support and insightful guidance in the course of this study.

I would like to thank my co-supervisor Professor M.R. Kianoush for his encouragement and advice that greatly helped me through this work.

The financial support of NSERC through Professor A. Varvani-Farahani of Department of Mechanical Engineering- Ryerson University as well as a partial support of Civil Engineering Department through Professor M.R. Kianoush are greatly appreciated.

Finally, I would like to thank all my fellow graduate students and family members for their support, encouragement and constructive criticisms. 
To my dear late grandmother

Parvati 


\section{TABLE OF CONTENTS}

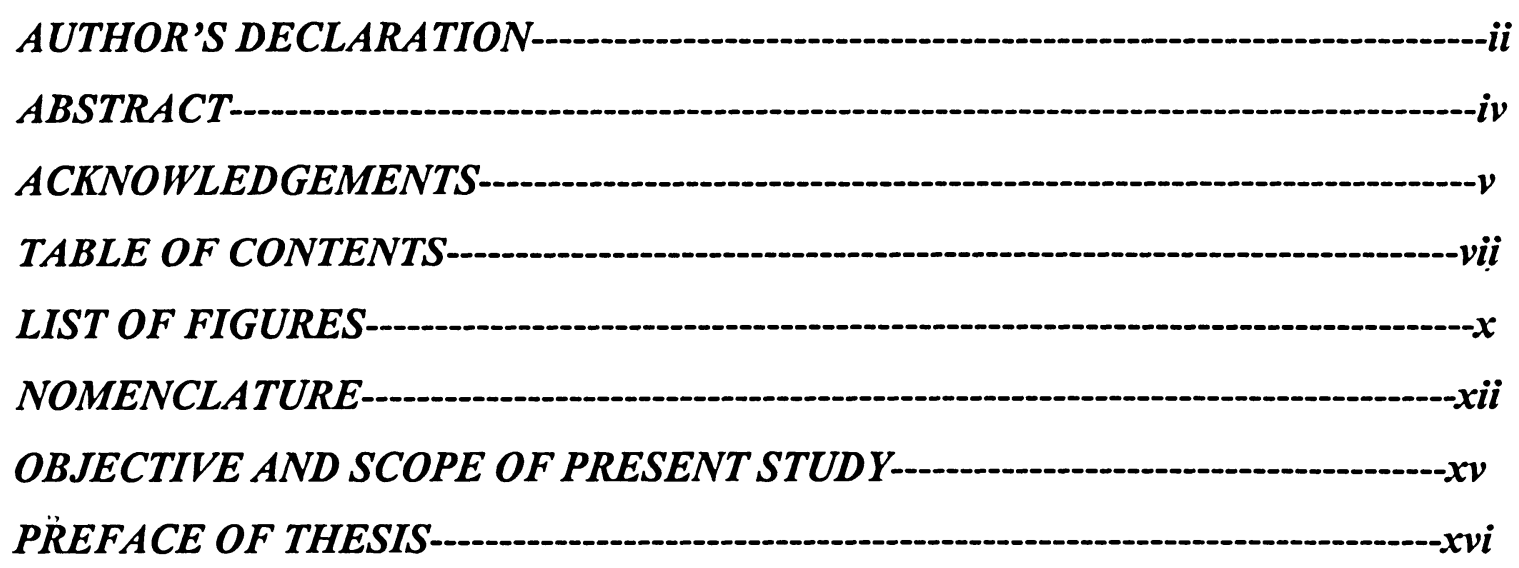

\section{Chapter 1: Introduction and Background}

1.1 Introduction$-1$

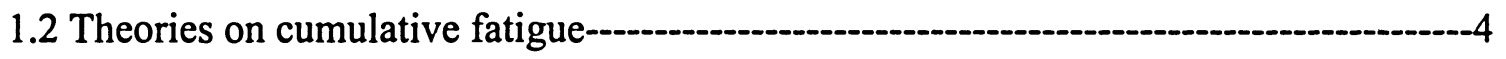

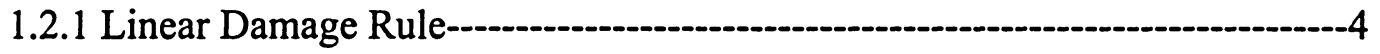

1.2.2 Non - Linear Theory--

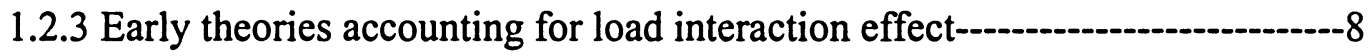

1.2.3.1 Two stage linear damage theories--

1.2.4 Life Curve Modification Methods - 10

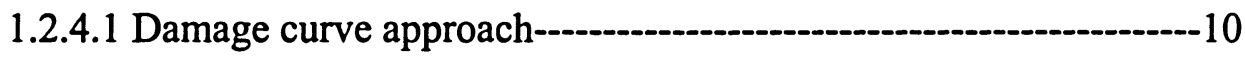

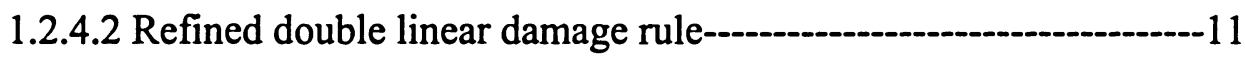

1.2.4.3 Double-damage curve approach--

1.2.4.4 Modified version to account for load interaction effect-----------12

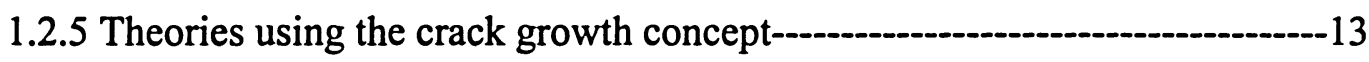

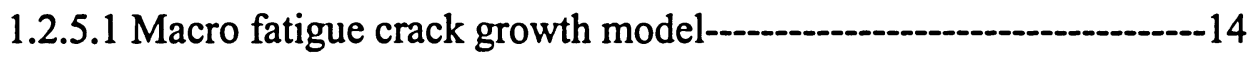

1.2.5.2 Double exponential law-14

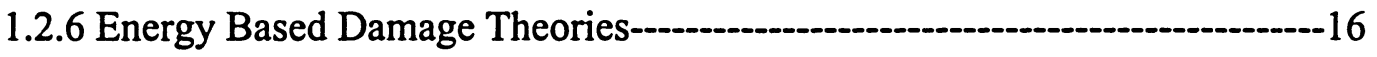

1.2.7 Continuum Damage Mechanics Approaches-

1.2.8 Critical plane Damage Theories--19

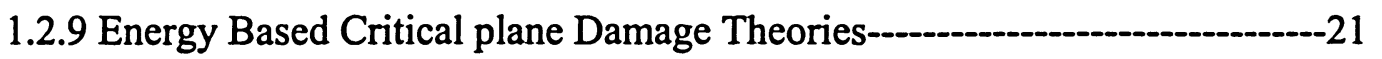




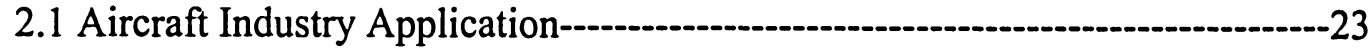

2.2 Automobile Industry Application--

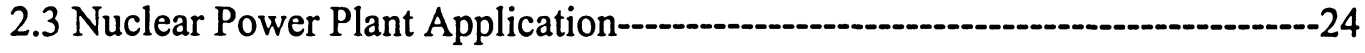

2.4 Civil Application-24

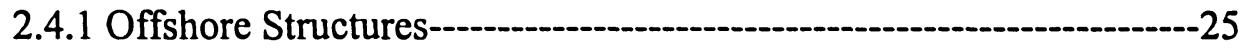

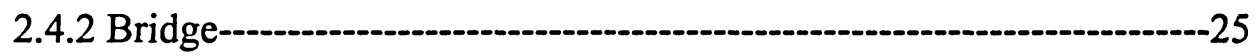

2.4.2.1 Steel bridge Deck-1-

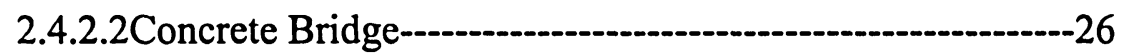

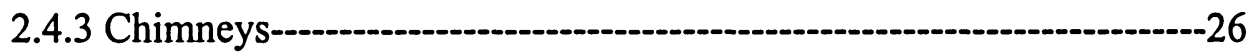

2.4.4 Pile Supported Structures--

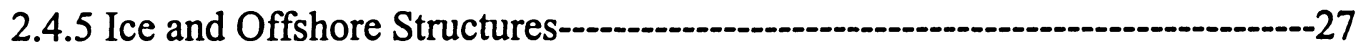

Chapter 3: Fatigue Damage Analysis and Modeling

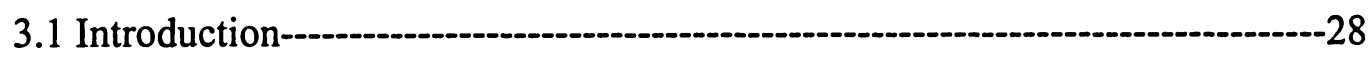

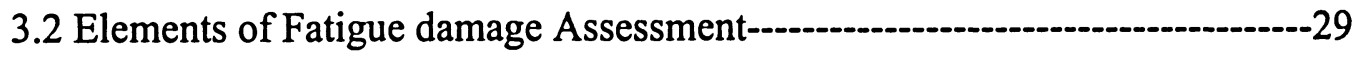

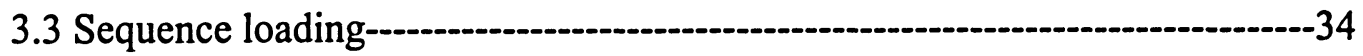

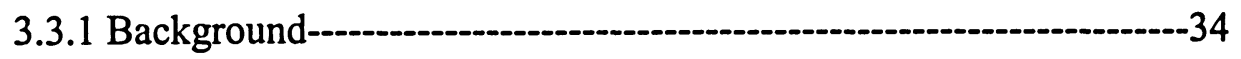

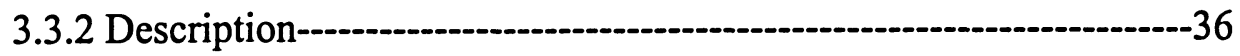

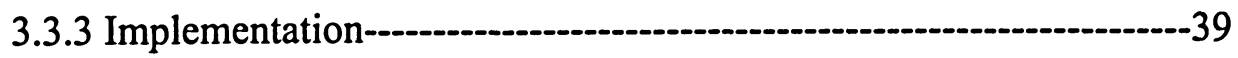

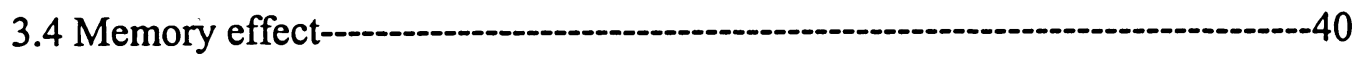

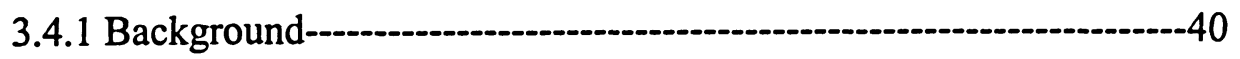

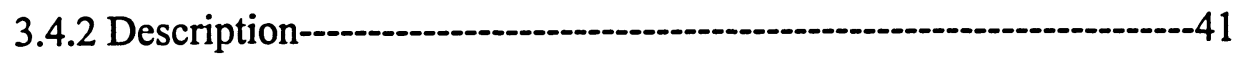

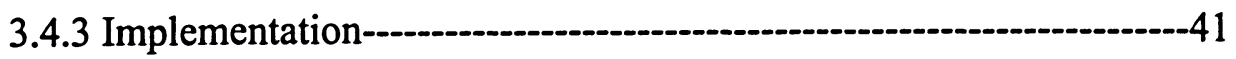

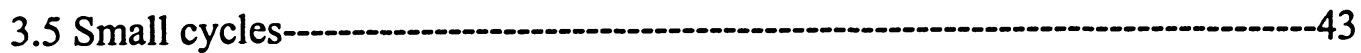

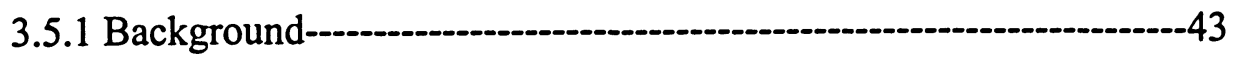

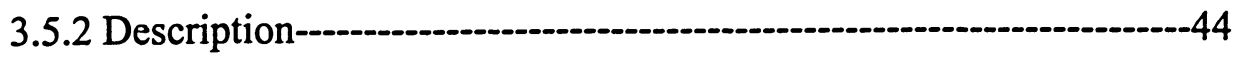

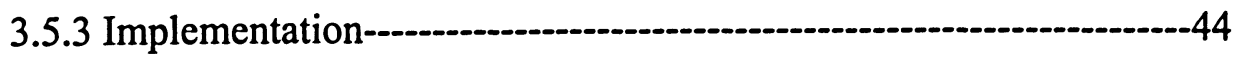


Chapter 4: Algorithm of fatigue damage Analysis $-50$

4.1 Description of Algorithm-

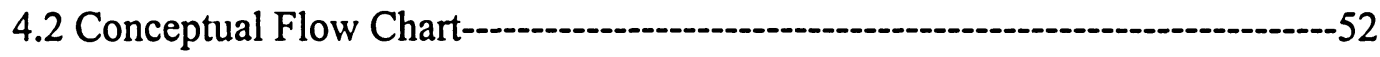

4.3 Computer program Flow Chart--

Chapter 5: Fatigue damage model evaluation and Results-

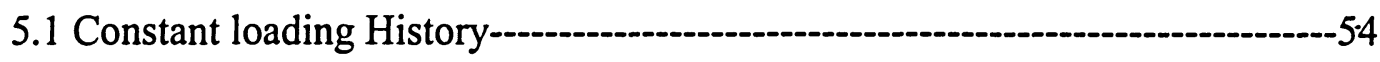

5.2 Step loading History--

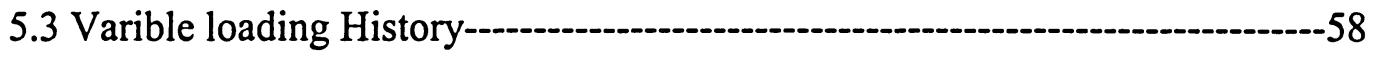

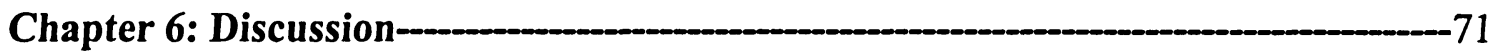

Chapter 7: Conclusion and Recommendations -

7.1 Conclusions--

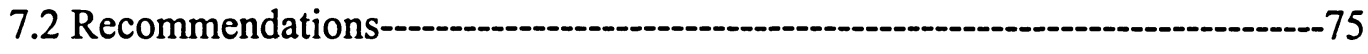

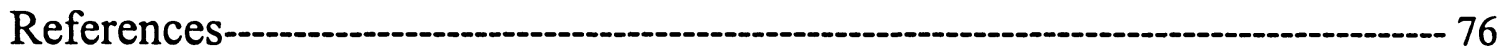

Appendix A: Material Property and data--an

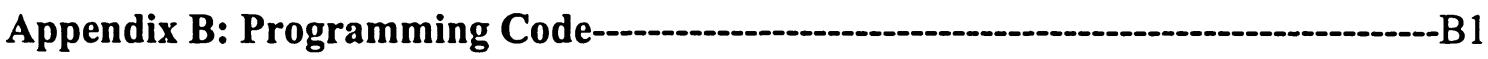




\section{LIST OF FIGURES}

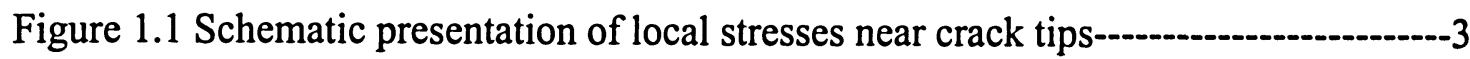

Figure 1.2 Effect of Miner's rule on S-N Curve--

Figure 1.3 Demonstration of Non-linear theory--.---_-

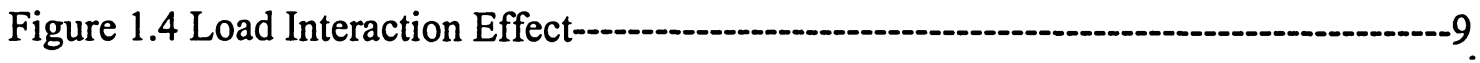

Figure 1.5 Comparison of DDCA with DLCR and DCA-12

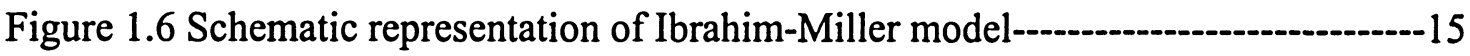

Figure 1.7 Energy Based Approach - Hysteresis Loop---_-

Figure 1.8 Schematic presentation of different Critical Planes----------------------------20

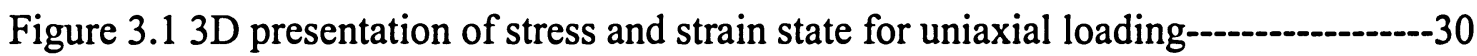

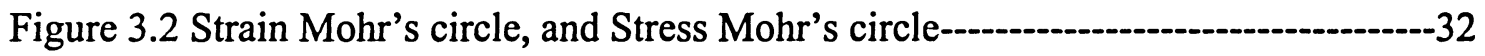

Figure 3.3 Steps of fatigue damage analysis and life prediction--

Figure 3.4 A schematic diagram of block loading sequence ----------------------------36

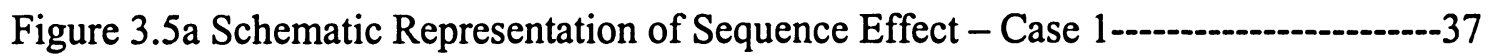

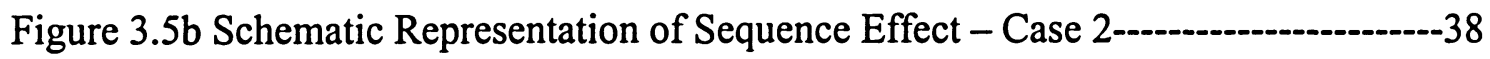

Figure 3.6 Schematic Representation of Memory Effect---o-a-

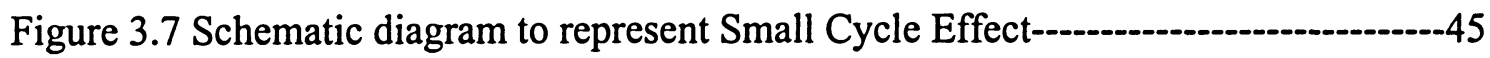

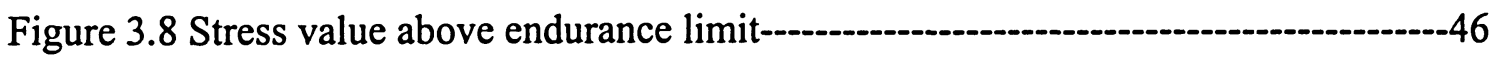

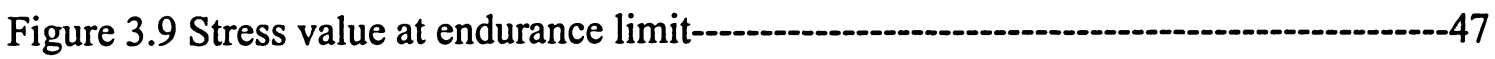

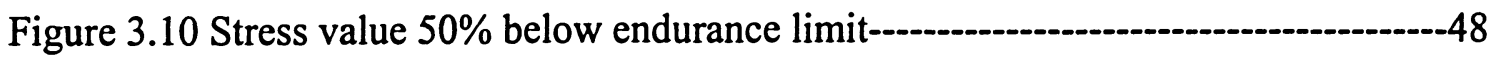

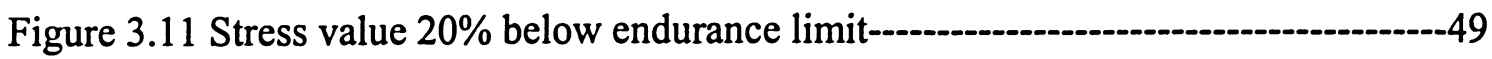

Figure 4.1 Conceptual flow chart---o-_-

Figure 4.2 Computer flow chart--.-.-.-- 53

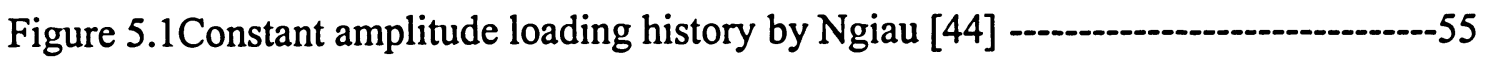

Figure 5.2 Comparison of Experimental data, new approach and Miner's rule-------------56

Figure 5.3 Experimental and predicted fatigue lives in Al 2024-T351alloy----------------57

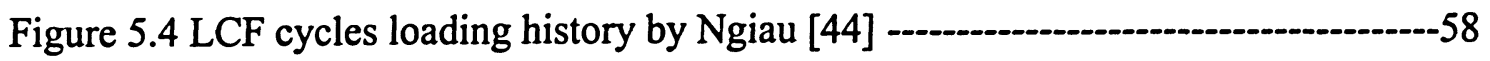

Figure 5.5 HCF cycles loading history by Ngiau [44] - -

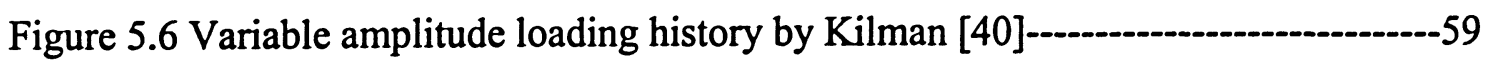

Figure 5.7 Comparison of Experimental data, new approach and Miner's rule-------------60 
Figure 5.8 Experimental and calculated fatigue lives for low carbon steel-o-a-a-a--- 61

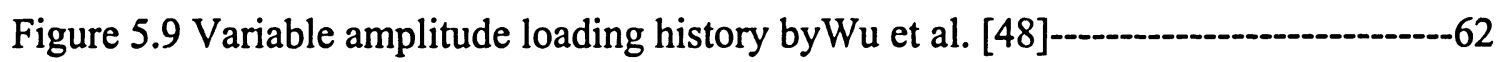

Figure 5.10 Comparison of Experimental data, new approach and Miner's rule-----------63

Figure 5.11 Experimental and calculated lives data for Al 7075-T 761 alloy --o-c--o-64

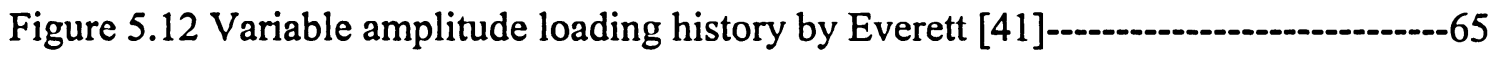

Figure 5.13 Comparison of Experimental data, new approach and Miner's rule-----------66

Figure 5.14 Experimental and calculated lives data for Al 2024 T3 alloy -----------------67

Figure 5.15 Variable amplitude loading history by Agerskov [47]--

Figure 5.16 Comparison of Experimental data, new approach and Miner's rule-----------69

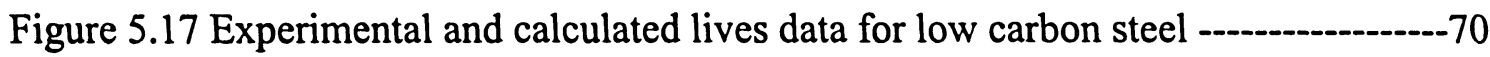




\section{NOMENCLATURE}

ASME - $\quad$ American Society of Mechanical Engineering

CPE - Critical Plane Energy

DCA - Damage Curve Approach

DLDR- Double Linear Damage Rule

DDCA- Double-Damage Curve Approach

E - Modulus of elasticity

HCF - High cycle fatigue

LCF - Low cycle fatigue

S-N - Stress -Life

$\epsilon-N \quad-\quad$ Strain-Life

LDR - Linear Damage Rule

MSC - Microstructurally short crack

PSC - Physically small crack

CAL - Constant amplitude loading

VAL - Variable amplitude loading

S - Alternating stress

K - $\quad$ Cyclic strength coefficient

$\mathrm{n}_{1} \quad-\quad$ Cyclic hardening exponent

$\mathrm{n}^{*} \quad$ - Constant unifies shear and tensile fatigue data.

$\mathrm{k} \quad$ - $\quad$ mathematical exponent to give close fit to the double linear damage rule.

$\mathrm{N}_{\mathrm{f}} \quad-\quad$ Cycles to failure

$\mathrm{N}_{\mathrm{r}}$. Number of cycle at reference level.

$\mathrm{D}_{\mathrm{i}} \quad$. Damage fraction

$\mathrm{c}_{\mathrm{i}}$. Retardation factor.

d - Material sensitivity constant.

p - Constant measured from the slope of the first damage accumulation line.

$\mathrm{p}_{1} \quad$ - Empirical shaping parameter depending on the material properties.

A - $\quad$ Material constant for refined double linear damage rule.

$\beta \quad$ Material constant (slope of regression line). 
$\beta_{1}, \beta_{2}, \beta_{3} \quad$ Material constant determined experimentally.

$\Delta \lambda, \Delta \lambda^{*} \quad$ Sequenced related parameters.

$a_{0}, a, a_{f} \quad$ Initial, Instantaneous and Final crack length.

$N_{1}, N_{11} \quad$ Two stages of damage linear rule.

$\Delta \varepsilon_{a p}, \Delta\left(\frac{\gamma_{a p}}{2}\right)$ The shear and axial strain ranges respectively.

$\Delta \varepsilon_{n}, \Delta\left(\frac{\gamma_{\max }}{2}\right)$ Maximum shear strain range and normal strain range acting on the critical plane, respectively.

$\Delta \sigma_{a}, \Delta \tau_{a}, \Delta \varepsilon_{a}$ Applied tensorial strain range, stress range, and shear stress range, respectively

$\Delta \tau_{\max }, \Delta \sigma_{n} \quad$ Maximum shear stress range and normal stress range, respectively

$\varepsilon_{1}, \varepsilon_{2}, \varepsilon_{3} \quad$ Principal strains $\left(\varepsilon_{1}>\varepsilon_{2}>\varepsilon_{3}\right)$

$\varepsilon_{e}, \varepsilon_{p} \quad$ Elastic strain and plastic strain respectively

$v_{e}, v_{p}, v_{e f f} \quad$ Elastic, plastic, and effective Poisson's ratios respectively

$\sigma_{1}, \sigma_{2}, \sigma_{3} \quad$ Principal stresses $\left(\sigma_{1}>\sigma_{2}>\sigma_{3}\right)$

$\sigma_{n}{ }^{m} \quad$ Mean normal stress

$\alpha \quad$ Life fraction factor for the initiation stage.

$\alpha_{1} \quad$ Material constant determined from regression analysis experimentally

$\lambda_{1}, \lambda_{2} \quad$ First and second fictitious load levels.

$\sigma_{n}{ }^{\max } \quad$ Maximum normal stress

$\sigma_{n}^{\min } \quad$ Minimum normal stress

$\sigma_{\max } \quad$ Maximum stress amplitude

$\sigma_{f}{ }^{\prime}, \varepsilon_{f}{ }^{\prime} \quad$ The axial fatigue strength coefficient and axial fatigue ductility coefficient, respectively.

$\tau_{f}{ }^{\circ}, \gamma_{f}{ }^{\circ} \quad$ The shear fatigue strength coefficient and shear fatigue ductility coefficient, respectively. 
$\varepsilon_{a 1}, \varepsilon_{a 2}, \varepsilon_{a 3} \quad$ Applied strain amplitudes in a step loading conditions $\left(\varepsilon_{a 1}>\varepsilon_{a 2}>\varepsilon_{a 3}\right)$. $\Delta \gamma_{p} \quad$ Plastic strain range. 


\section{OBJECTIVE AND SCOPE OF PRESENT STUDY}

The present study intends to further apply a recently developed Varvani's fatigue damage parameter to assess the fatigue damage of smooth 1045 steel components subjected to repeated variable block histories. The parameter is the sum of the normal energy range and shear energy calculated for critical plane on which the stress and strain Mohr's circles are largest during each peak-valley cyclic loads. Fatigue damage has been accumulated on the basis of peaks and valleys throughout the variable block loading histories using a computer algorithm developed in this study. In fatigue damage assessment under variable loading conditions, further phenomenological factors of (i) loading sequence effect, (ii) the effect of small amplitude cycles below the materials endurance limit, and (iii) hysteresis memory effect have been taken into account.

The effect due to sequence loading is studied for variable loading. It is found that the loading sequence has a great influence in the stress-strain response of hysteresis loops. Memory effect concept has been carefully monitored and programmed to correspond to the closed hysteresis loop in each block loading history. The small amplitude cycles corresponds to $50 \%$ to $100 \%$ of the materials endurance limit are also studied. Results indicate that small cycles exceeding $50 \%$ of the fatigue endurance limit contribute to the accumulated damage. The variation of mean stress as peaks and valleys of a block loading history fluctuate has been included in fatigue damage calculation.

A comparison of the predicted fatigue life results based on energy based-critical parameter including the phenomenological factors with the experimental fatigue life data has been found in a good agreement. 


\section{PREFACE OF THESIS}

The following provides a brief description of material covered in this thesis. This discussion emphasizes practical application, evaluation of fatigue damage assessment subjected to variable amplitude loading conditions.

Chapter 1 covers the introduction and background of three basic approach stress, strain and fracture mechanics. The chapter discusses about early fatigue damage model such as well-known Miner's rule, non-linear damage rule. It also covers the early approaches developed in1970's to some of the latest models developed such as critical plane damage theories, continuum damage mechanics approaches, energy based-critical plane damage approaches.

Chapter 2 reviews the practical application in the field of aeronautics, automobile and civil industries (such as bridge, chimney and offshore structures). These applications will give the brief idea on how fatigue damage analysis is used in the diverse engineering fields.

Chapter 3 discusses Varvani's fatigue damage approach and includes all terms required for damage assessment using this approach. This chapter further discusses the states of stress and strain and phenomenological factors such as sequence effect, memory effect and small cycle effect, for fatigue damage assessment under variable amplitude loading conditions.

Chapter 4 addresses the detailed description of computer method developed for the analysis of fatigue damage model. It also describes detailed steps involved in computer program, for Varvani's parameter as well as phenomenological factors.

Chapter 5 evaluates the damage analysis results for various fatigue data available in the literature. The model is tested with diverse engineering applications in order to prove the 
applicability of the approach. The experimental data referenced from literature are explained in detail because each data set corresponded to specific loading conditions.

Chapter 6 discusses the advantages of a new proposed model and comparison of a new proposed approach with other critical plane approaches.

Chapter 7 summarizes the conclusions obtained by evaluating the fatigue damage model. It gives details about how accurately this approach can be used for variable loading conditions. It also includes recommendations for researchers who are interested to carry out further studies on the topic. 


\section{CHAPTER ONE}

\section{Introduction and Background}

\subsection{Introduction:}

Fatigue failures are common modes of failure observed in various mechanical components and structures. In real engineering applications where fatigue is an important failure mode, the alternating stress amplitude usually changes in irregular manner. Proper prediction of life of those objects is a very important problem and any underestimation can cause catastrophic failure.

Fatigue is a process which causes premature failure or damage of component subjected to repeated loading. It is a complicated metallurgical process to accurately describe and model on the microscopic level. Despite this complexity, fatigue analysis methods have been developed. The three primary fatigue analysis methods are stress-life approach, the strain-life approach, and the fracture mechanics approach.

(i) The Stress - Life (S-N) approach: This method was the first approach used in an attempt to understand and quantify metal fatigue. It was the standard fatigue design method for almost 100 years. The S-N approach is still widely used in design applications where the applied stress is primarily within the elastic range of the material and resultant lives are long. The basis of the stress-life method is the S-N diagram, which is a plot of alternating stress, $\mathrm{S}$, versus cycles to failure, $\mathrm{N}_{\mathrm{f}}$. This method does not work well in low-cycle applications. The dividing line between the low and high cycle fatigue depends on the material being considered, but usually falls between 10 and $10^{5}$ cycles. One of the major drawbacks of the stress-life approach is that it ignores true stress-strain behavior and treats all 
strains in the elastic range. This method is completely empirical in nature and lacks the physical insights. The plastic strains, which are critical at the short lives, are ignored and at long lives most of steels have only a small plastic component of cyclic strain. The S-N approach does not distinguish between crack initiation and propagation stages.

(ii) Strain-Life ( $\epsilon-\mathrm{N})$ Approach: This method takes into account actual stress-strain response of the material, plastic strain, and the mechanism that is modeled and is used in high strain/low cycle regime. In most engineering components [1], the response in critical locations is either dependent on strain or deformation. When the load levels are low, stresses and strains are linearly related. At high load levels [1], in the low cycle fatigue (LCF) regime, the cyclic stress-strain response and material behavior are best modeled under strain-controlled condition. Fatigue research showed [1] that damage is dependent on plastic deformation or strain. In the strain-life approach the plastic strain or deformation is directly measured and quantified. The strain-life method assumes that smooth specimen tested under strain-controlled can stimulate fatigue damage at the notch root of an engineering component. Crack growth is not explicitly accounted for in the strain-life method. This method can model the residual mean stresses resulting from the sequence effect in load histories. This allows for more accurate accounting of cumulative damage under variable amplitude loading. It is used in high temperature application where fatigue creep interaction is critical. This method involves a more complicated level of analysis like Neuber analysis, finite element analysis or strain gauge measurement.

(iii) Fracture Mechanics Approach: The fatigue life of a component comprises of two stages: initiation and propagation stages. The size of the crack at the transition from initiation to propagation is usually unknown and often depends on the point of view of the analyst. At low strain amplitude [1], about $90 \%$ of the life is spent at initiation stage, while at high amplitude the majority of the fatigue life may be spent propagating a crack. Fracture mechanics approaches are used to estimate the propagation life. It requires an initial crack size be known for component with imperfections or defects, such as welding porosities, inclusions and casting defects, etc. Linear elastic fracture mechanism 
principles are used to relate the stress magnitude and distribution near the crack tip to remote stress applied to the cracked component, the crack size and shape, the material properties of the crack component. Griffith [2] formulated the concept that a crack in a component will propagate if the energy is lowered with crack propagation the total energy of the system is lowered. Irwin [3] extended the theory for ductile materials. He postulated that the energy due to plastic deformation must be added to the surface energy associated with the creation of new crack surfaces. He recognized that for ductile materials, the surface energy term is often negligible compared to the energy associated with plastic deformation. Irwin [4] made another significant contribution, which states that the local stresses near the crack tip are of the general form as shown in the Figure 1.1 .
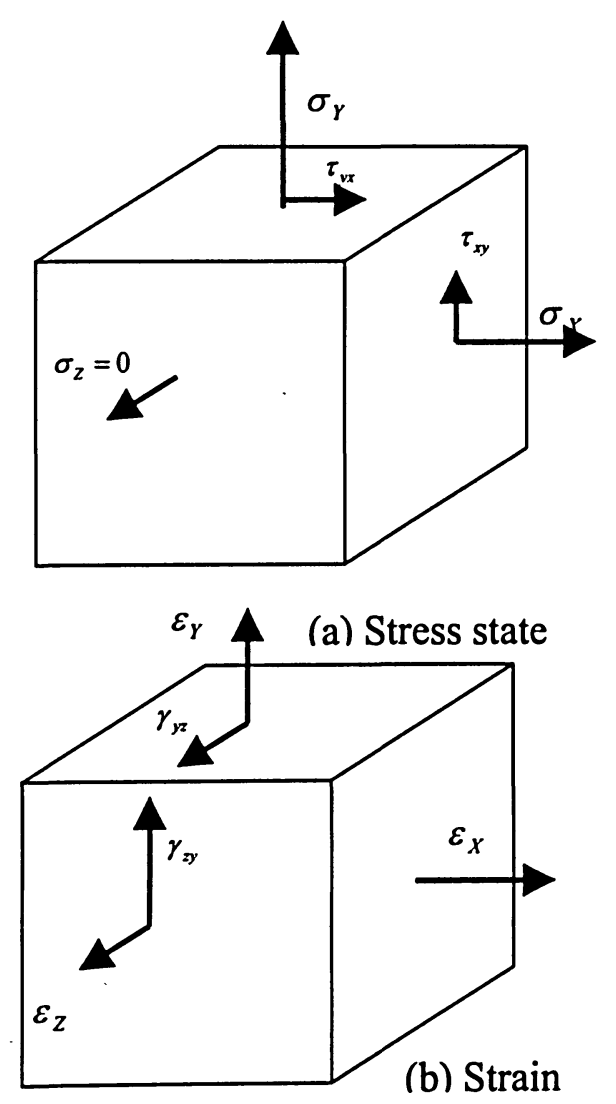

(b) Strain

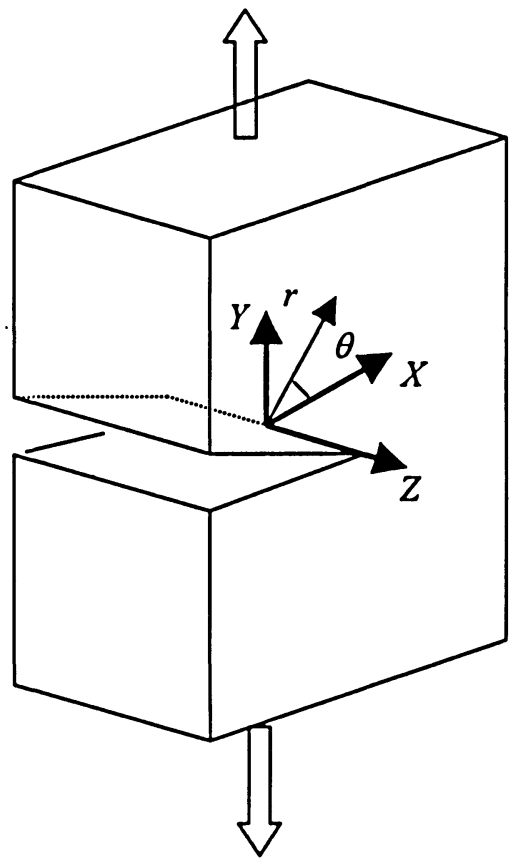

(c) local stresses near crack

Figure 1.1 Schematic presentation of local stresses near crack tips for plane stress condition. 
Historically, two considerations have promoted the development of fatigue analysis methods. The first has been the need to provide designers and engineers with methods that are practical and easily implemented, and cost effective. The second consideration has been the need to reconcile these analytical considerations with physical considerations. Economics of time and money is an important consideration when selecting an analysis technique. The S-N approach is the quickest and cheapest of the approaches, but the advantages of the other methods may far outweigh cost consideration. Any of the fatigue life estimation techniques can be used for either initial sizing or design of new component or for the analysis of an existing component.

\subsection{Theories on cumulative fatigue damage:}

Several researchers have reviewed theories on cumulative fatigue damage and divided these theories into the following categories:

1. Linear damage rules.

2. Non-linear damage curve.

3. Two stage linearization approaches.

4. Life curve modification methods.

5. Approaches based on crack growth concepts.

6. Energy based theories.

7. Continuum damage mechanics model.

8. Critical plane Damage theories, and

9. Energy-based critical plane damage theories.

\subsubsection{Linear damage rules (LDR):}

The first cumulative damage rule was proposed by Palmgren [5] in 1924 and later developed by Miner [6] in 1945. This linear theory, which is still widely used now, is referred to as the Palmgren-Miner rule or Miner's rule. 
It simply states that fatigue failure is expected when the summation of all the fatigue damage caused by different stress level reaches unity.

$$
\sum_{i=1}^{n} \frac{n}{N_{f}}=\text { Cycle ratio }
$$

where $n$ and $N_{f}$ are the number of cycles and the fatigue life in cycles at stress level S respectively.

The damage fraction, D, is defined as the fraction of life used up by an event or a series of events. Failure in any of the cumulative damage theories is assumed to occur when the summation of damage fraction equals 1 :

$$
\sum D_{i}=1
$$

The linear damage rule states that the damage fraction, $D_{i}$, at stress level $S_{i}$ is equal to the cycle ratio $n_{i} / N_{i .}$.

$$
D_{i}=\sum \frac{n_{i}}{N_{i}}=1
$$

Linear damage rules cannot account for load sequence and load interaction effect due to their linear nature. The Linear damage rule has two main shortcomings when it describes observed material behavior. First, it does not consider sequence effect. The linear damage rule does not hold a term to show the effect of stress amplitude. Miner's rule can also be interpreted graphically by showing its effect on the S-N curve as shown in Figure 1.2. 


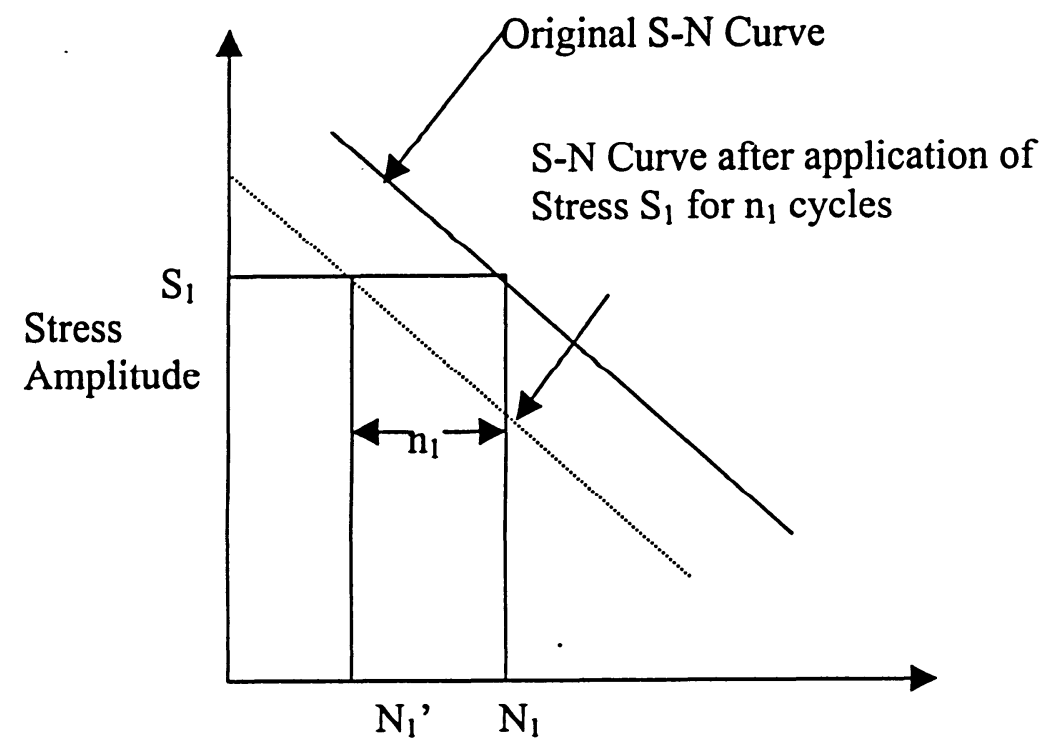

Cycle to failure

Figure 1.2 Effect of Miner's rule on S-N Curve [1].

\subsubsection{Non-Linear Theories:}

Many nonlinear damage theories have been proposed which attempt to overcome the shortcomings of Miner's rule. There are some practical problems involved when trying to use these methods. Firstly, they require material constants, which must be determined from a series of step tests. This requires a considerable number of tests to conduct. Secondly, since some of the methods take into account sequence effects, the number of calculations and lengthy procedure can become a problem in complicated histories. Another point is that although the nonlinear methods may give better predictions than Miner's rule for two-step histories, it cannot be guaranteed that they will work better for actual service load histories.

Macro and Starkey [7] proposed the first non-linear load dependent damage theory in 1954 , represented by a power relationship as: 


$$
D=\sum r_{i}^{x_{i}}
$$

where $x_{i}$ is a variable quantity related to the $i^{\text {th }}$ loading level. The concept of change in endurance limit due to pre-stress exerted an important influence on subsequent cumulative fatigue damage research. Kommers [8] and Bennelt [9] further investigated the effect of fatigue pre-stressing on endurance properties using a two-level step loading method. Their experimental results suggested that the reduction in the endurance strength could be used as a damage measure, but they did not correlate this damage parameter to the life fraction. All of these damage models based on endurance limit reduction are nonlinear and able to account for the load sequence effect. None of these models, however, take into account the interaction effect. The use of above method is shown in Figure 1.3, which is plot of damage fraction versus cycle ratio for two stress levels, where $S_{1}>S_{2}$ and damage calculation is done along $\mathrm{OA}$ ' $\mathrm{AB}$ (dotted line shown in the Figure).

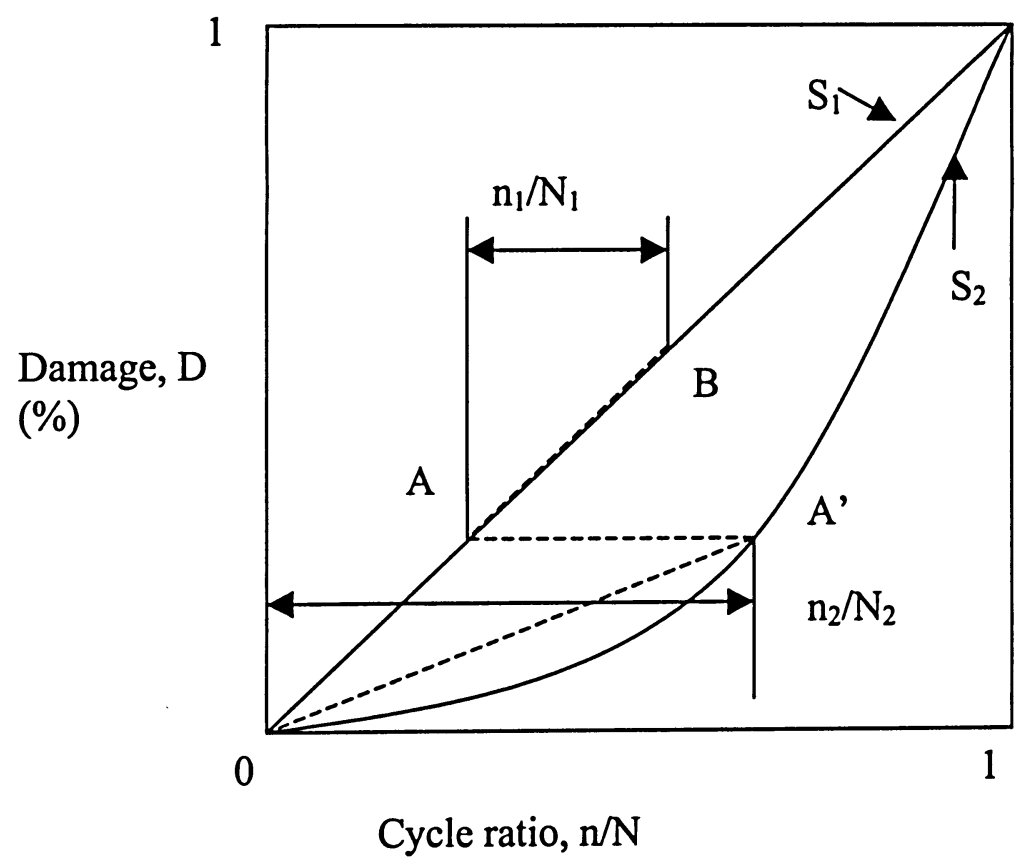

Figure 1.3 Demonstration of Non-linear theory [1]. 


\subsubsection{Early theories accounting for load interaction effect:}

Splitzer-Corten [10] and Freudenthal-Heller [11] approaches are based on the modification of the S-N diagram, which is simply a clockwise rotation of S-N line around a reference point on the line. In Splitzer-Corten model the reference point corresponds to the highest level, while in Freudenthal-Heller approach, this reference is chosen at the stress level corresponding to the fatigue life of $10^{3}-10^{4}$ cycles. Manson et al. [12] also examined the approach based on the S-N line rotation and convergence concept. They suggested that a point corresponding to failure life between $10^{2}$ and $10^{3}$ cycles on the original S-N line can be selected as the convergence point. Their approach also provides a method for predicting the reduction in endurance limit due to pre-cycling damage, and is therefore able to account not only for the load interaction effect, but also for small cycle damage. Marrow [13] has proposed a plastic work interaction damage rule which modifies Miner's rule by multiplying a given stress cycle ratio (the given stress to the maximum stress) to power factor to incorporate the interaction effect. The factor is called the plastic work exponent and can be interpreted as the material sensitivity to the variable-amplitude stress history. According to Marrow, when a component is subjected to variable-amplitude loading, the damage accumulation is given by the following:

$$
D=\sum_{i=1}^{n} \frac{n_{i}}{N_{f i}}\left(\frac{\sigma_{i}}{\sigma_{\max }}\right)^{d}
$$

in which $N_{f i}$ indicates the number of cycles to failure when the specimen or component is subjected to strain of amplitude $\varepsilon_{i}$ (or stress amplitude $\sigma_{i}$ ), $n_{i}$ is the applied strain cycle of $\varepsilon_{i}$ (or stress cycles of $\sigma_{i}$ ), $\sigma_{\max }$ is the maximum stress amplitude among all stress amplitude applied to the component, $\mathrm{d}$ is the material's sensitivity to the variable amplitude loading. It is noted that equation (1.5) reduces to equation (1.3) when $d=0$, which indicated that Palmgren-Miner's linear damage rule is a special case of the proposed non-linear damage rule. Figure 1.4 shows the schematic representation for twolevel L-H and H-L stressing. In these Figures, solid lines represent the Miner rule, dash line represents rotation from S-N curve to LDR, which is parallel to the original S-N 
curves. It can be seen that the LDR and the S-N line rotation approaches differ in their abilities to account for the load interaction effects.

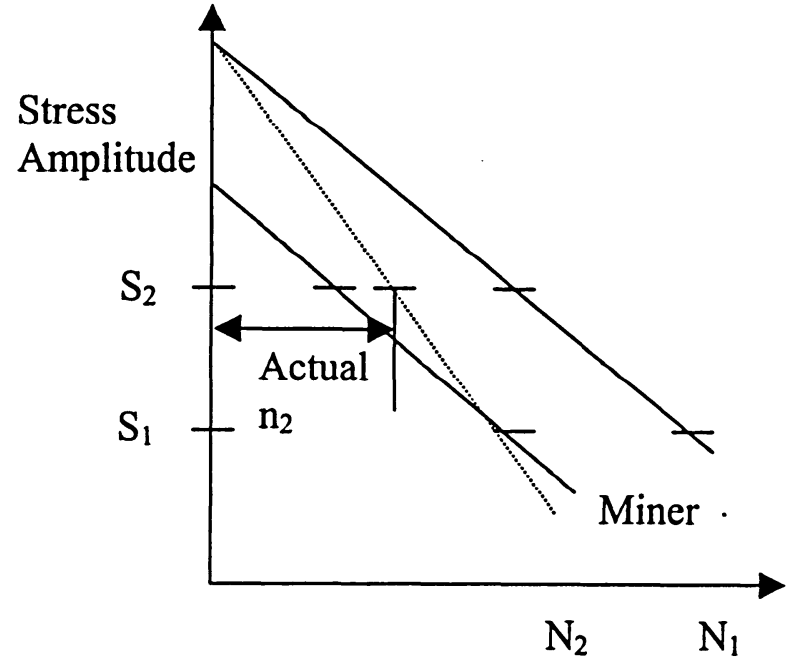

Cycle to failure (log scale)

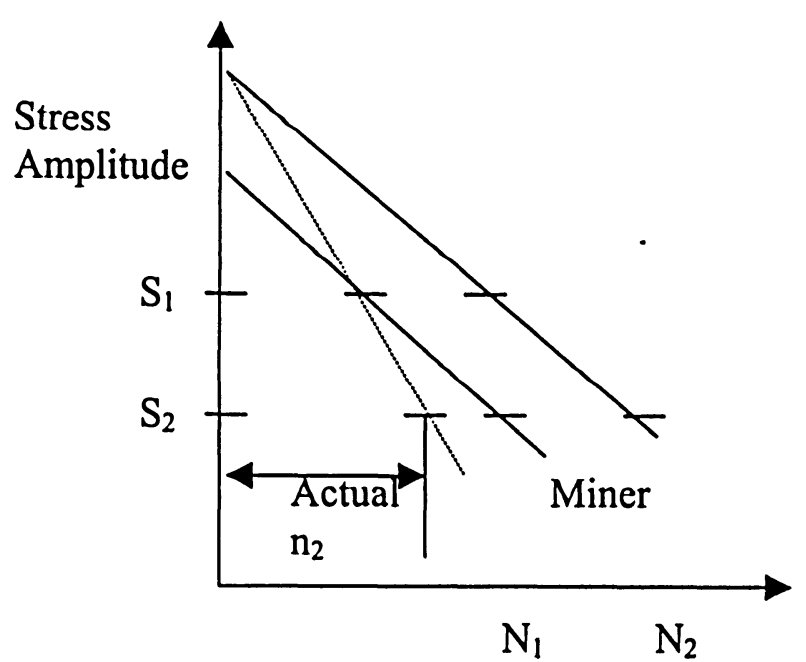

Cycle to failure (log scale)

(a) H-L load Sequence

(b) L-H load Sequence

Figure 1.4 Load Interaction Effect [12].

\subsubsection{Two stage linear damage theories:}

The two-stage damage approach improves on the LDR shortcomings, while still retains its simplicity in form. Grover [14] considered cycle ratio for two separate stages in the fatigue damage process of constant amplitude stressing:(i) Damage due to crack initiation $N_{1}=\alpha N_{f}$; and (ii) Damage due to crack propagation $N_{11}=(1+\alpha) N_{f}$. The term $\alpha$ is the life fraction factor for the initiation stage and $N_{f}$ is fatigue life in cycles. In two stage linearization approaches, the damage process is divided into two stages of crack initiation and crack propagation and the LDR is applied in each stage. Later, Mason [15] reverted to Grover's work and proposed the double linear damage rule (DLDR). In DLDR, the two stages were separated by equations of $N_{1}=N_{f}-P N_{f}^{0.6}$ and $N_{11}=P N_{f}^{0.6}$ where $P$ is a coefficient of the second stage fatigue life. 


\subsubsection{Life Curve Modification Methods:}

\subsubsection{Damage curve approach (DCA):}

This approach was developed to refine DDLR through a reliable physical basis. It is recognized that the major manifestation of damage is crack growth, which involves many complicated processes such as dislocation, agglomeration, sub cell formation, multiple crack formation and independent growth of these cracks until they link and form a dominant crack. Mason and Halford [16] empirically formulated the 'effective crack growth' model that accounts for the effects of these processes. This model is represented as:

$$
a=a_{0}+\left(a_{f}+a_{0}\right) \sigma^{q}
$$

where $a_{0}, a_{,} a_{f}$ are initial (at which $\mathrm{r}=0$ ), instantaneous and final (at which $\mathrm{r}=1$ ) crack lengths, respectively. The exponent $q$ is defined as $q=B N^{\beta}$ where $B$ and $\beta$ are two material constants. Damage is then defined as the ratio of instantaneous to final crack length, $D=\frac{a}{a_{f}}$. In most cases $a_{0}=0$, and the damage function of DCA becomes:

$$
D=r^{q}
$$

This form is similar to Marco-Starkey theory [7] equation (1.4). Through a series of two level tests, the constant $\beta$ can be determined from the slope of the regression line of the experimental data. If a reference level, $N r$, is selected, the other constant, $\mathrm{B}$, can then be expressed as $N_{r}^{-\beta}$. Therefore exponent q in equation (1.7) can be written as:

$$
q=\left(\frac{N_{f}}{N_{r}}\right)^{\beta}
$$

which is a load level dependent term. 


\subsubsection{Refined double linear damage rule (Refined DLDR):}

The original DLDR can be refined by linearization of damage rule curves defined by DCA model. In the refined DLDR, the knee points in a damage vs. cycle ratio (D-r) plot divide the damage process into two phases, and these two phases are determined by using below mentioned equations:

$$
D_{\text {knee }}=A\left(\frac{N_{r}}{N_{f}}\right)^{\alpha_{1}} \quad \text { And } \quad v_{\text {knee }}=1-(1-A)\left(\frac{N_{r}}{N_{f}}\right)^{\alpha_{1}}
$$

where $A$ and $\alpha_{1}$ are two constants determined from regression analysis of the experimental data [16]. The empirical values of these two constants were found to be $A=$ 0.35 and $\alpha_{1}=0.25$ for high strength steel.

\subsubsection{Double-damage curve approach (DDCA):}

This approach is developed by adding a linear term to the DCA equation with some mathematical manipulation and can be presented as:

$$
D=\left[(p r)^{k}+(1-p)^{k} r^{k q}\right]^{1 / k}
$$

where $\mathrm{k}$ is a mathematical exponent to give a close fit to the double linear damage line, and $p$ is a constant measured from the slope of the first damage accumulation line DLDR:

$$
P=\frac{D_{\text {knee }}}{v_{\text {knee }}}=\frac{A\left(\frac{N_{r}}{N_{f}}\right)^{\alpha_{1}}}{1-(1-A)\left(\frac{N_{r}}{N_{f}}\right)^{\alpha_{1}}}
$$

The DDCA represents a continuous damage curve, which conforms to the DLDR line in the early portion of phase I regime, but blends into DCA curve, which is also close to the DLDR in phase II. To evaluate the effectiveness of the developed DDCA, Mason et al. [17] conducted cumulative damage experiments on the both 316 stainless steel and 
Haynes Alloy 188. A comparison of their experimental results with DDCA predictions indicated good agreement. The above models possess similar characteristics. They are all load-level dependent; but do not account for the load interaction effect and smallamplitude cycle damage. Figure 1.5 shows the comparison between three methods.

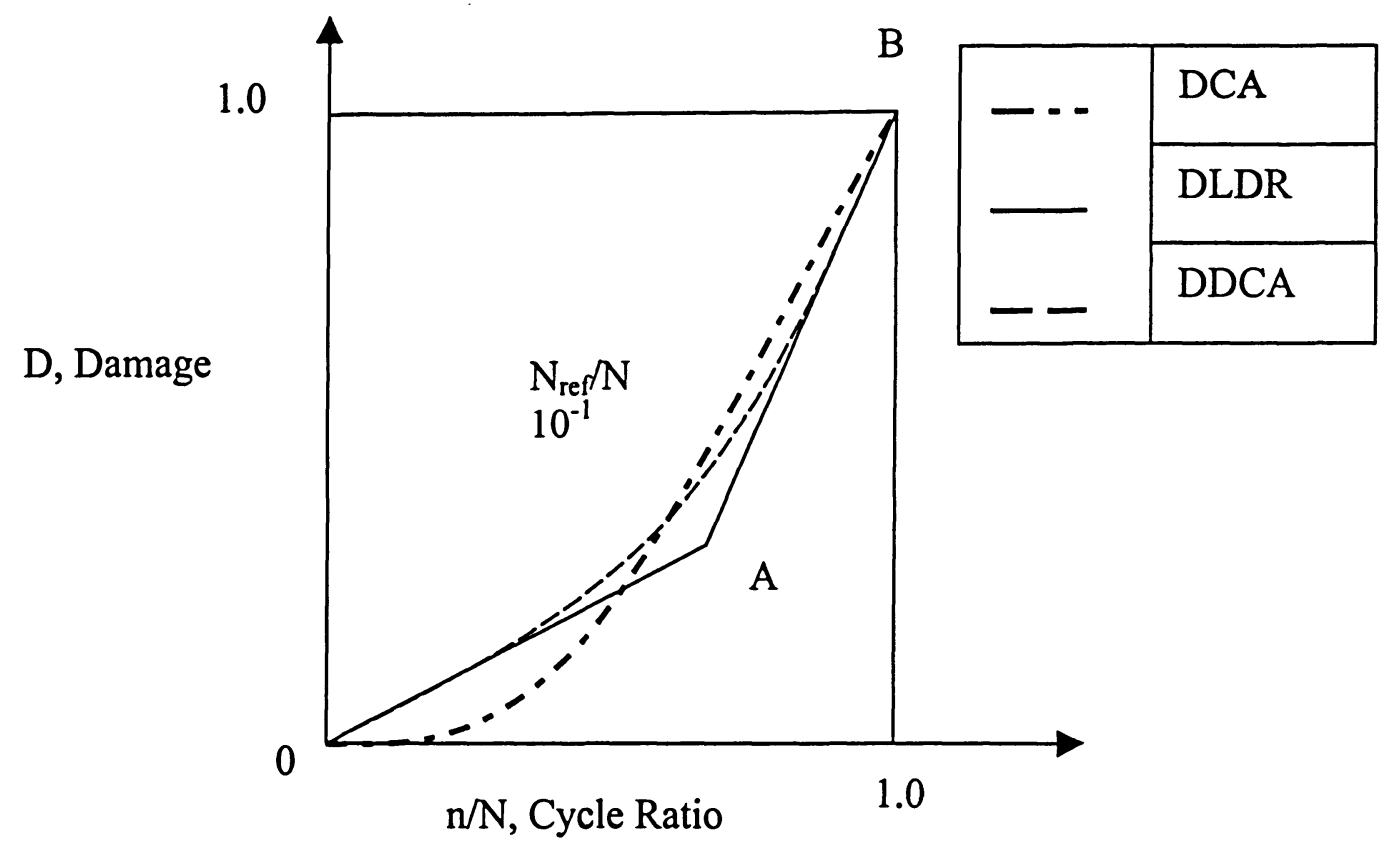

Figure 1.5 Comparison of DDCA with DLCR and DCA [17].

\subsubsection{Modified version to account for load interaction effect:}

To account for load interaction effect, Bui-Quoc[18] developed two approaches to improve the model. One is the fictitious load approach and another is the cycle ratio modification approach. The fictitious load approach was developed only for two-step load cycling. In this approach, there is no modification of the load parameter for first level $\lambda_{1}$. For the second load level; the load parameter $\lambda_{2}$ is replaced by an imaginary strain, therefore $\lambda_{2}$ are called 'fictitious load'. To determine the fictitious value $\lambda_{2}$, parameter $\mathrm{Y}$ used in regression analysis as:

$$
Y=1+\beta_{1}\left(\frac{|\Delta \lambda|}{\Delta \lambda^{*}}\right)^{\beta 2} r_{1}^{\beta 3}
$$


where $\beta_{1} \beta_{2} \beta_{3}$ are determined experimentally; $\Delta \lambda$ is the difference between strain levels $\Delta \lambda=\lambda_{2}-\lambda_{1} ; Y$ and $\Delta \lambda^{*}$ are sequence related parameters defined as follows:

for the Low-High increasing steps.

$$
\begin{aligned}
& Y=\lambda_{f}^{*}-\frac{\lambda_{2}}{\lambda_{f}^{*}}-\lambda_{2} \text { and } \Delta \lambda^{*}=\lambda_{f} \cdot \lambda_{1} \\
& \text { where } \lambda_{f} \cdot=\frac{8}{7} \lambda_{f} ;
\end{aligned}
$$

and for the High-Low decreasing steps.

$$
Y=\lambda_{2}-\frac{1}{\lambda_{2}}-1 \text {, and } \Delta \lambda^{*}=\lambda_{1}-1
$$

In the cycle ratio modification approach, introducing an exponent in stress/strain version [18] modifies the damage function equation, $v$, to the cycle ratio $r^{v}$ therefore, $v$ is called a load - interaction parameter. For two-step cycling, $v$ is related to another parameter, $\alpha_{1}$, by empirical equation of:

$$
v=\left[1-\left(\frac{|\Delta \lambda|}{\lambda_{f}-1}\right)^{\alpha_{1}}\right]^{\frac{\Delta \lambda}{|\Delta \lambda|}}
$$

where $\Delta \lambda=\lambda_{2}-\lambda_{1}$. The value of the material constant $\alpha_{1}$ is in the range of $0-1$. It can be experimentally determined from two-step fatigue test, or empirically estimated by taking $\alpha_{1}=0.5$ as a reasonable approximation.

\subsubsection{Theories using the crack growth concept:}

The crack growth concepts developed in 1950s and 1960s have been widely accepted. This is due to the fact that damage concept was defined based on cracking response of materials, and the development of technology provided sophisticated tools and techniques in measuring of very small cracks of the order of $1 \mu \mathrm{m}$. 


\subsubsection{Macro fatigue crack growth model:}

A popular macro fatigue crack growth retardation model is the wheeler model [19]. This model assumes the crack growth rate to be related to the interaction of crack-tip plastic zone under residual compressive stresses created by overloads. This model modifies the constant amplitude growth rate equation, $\frac{\mathrm{da}}{\mathrm{dN}}=\left[\mathrm{A}(\Delta \mathrm{k})^{\mathrm{m}}\right]$ by empirical retardation factor $c_{i}$.

$$
\frac{\mathrm{da}}{\mathrm{dN}}=\mathrm{c}_{\mathrm{i}}\left[\mathrm{A}(\Delta \mathrm{k})^{\mathrm{m}}\right] \text { Where } c_{i}=\left(\frac{r_{p i}}{r_{\max }}\right)^{p_{1}}
$$

where $r_{p i}$ is the plastic zone size associated with the $\mathrm{i}^{\text {th }}$ loading cycle, $\mathrm{r}_{\max }$ is the distance from the current crack tip to the largest prior elastic-plastic zone created by the overload, and $p_{1}$ is an empirical shaping parameter depending on material properties and load spectrum. Constants $\mathrm{A}$ and $\mathrm{m}$ are material constants. A similar retardation model based on the crack tip plasticity is the Willenburg model [16]. This model uses an effective stress intensity factor at the crack tip, $\left(\Delta k_{e f f}\right)$, which is caused due to the increased crack tip residual compressive stress induced by the overloads. The reduction in the applied $(\Delta k)$ is a function of the instantaneous plastic zone size at the $\mathrm{i}^{\text {th }}$ load cycle and if the maximum plastic zone at the $i^{\text {th }}$ load cycle

\subsubsection{Double exponential law:}

For the accumulation of fatigue damage in crack initiation and stage I growth, Miller and Zachariah [21] introduced an exponential relation between the crack length and elapsed life for each phase. The approach is thus termed double exponential law. In this model damage is normalized as $D=\frac{a}{a_{f}}$, where $a$ and $a_{f}$ are instantaneous and final crack lengths respectively. Later, Ibrahim and Miller [22] significantly modified this model. Based on the growth mechanism of very small cracks, propagation behavior in stage I was then mathematically described in a manner similar to that expressed by LEFM for stage II growth as:

$$
\frac{d a}{d N}=\phi\left(\Delta \gamma_{p}\right)^{\alpha_{1}} \alpha_{1}
$$




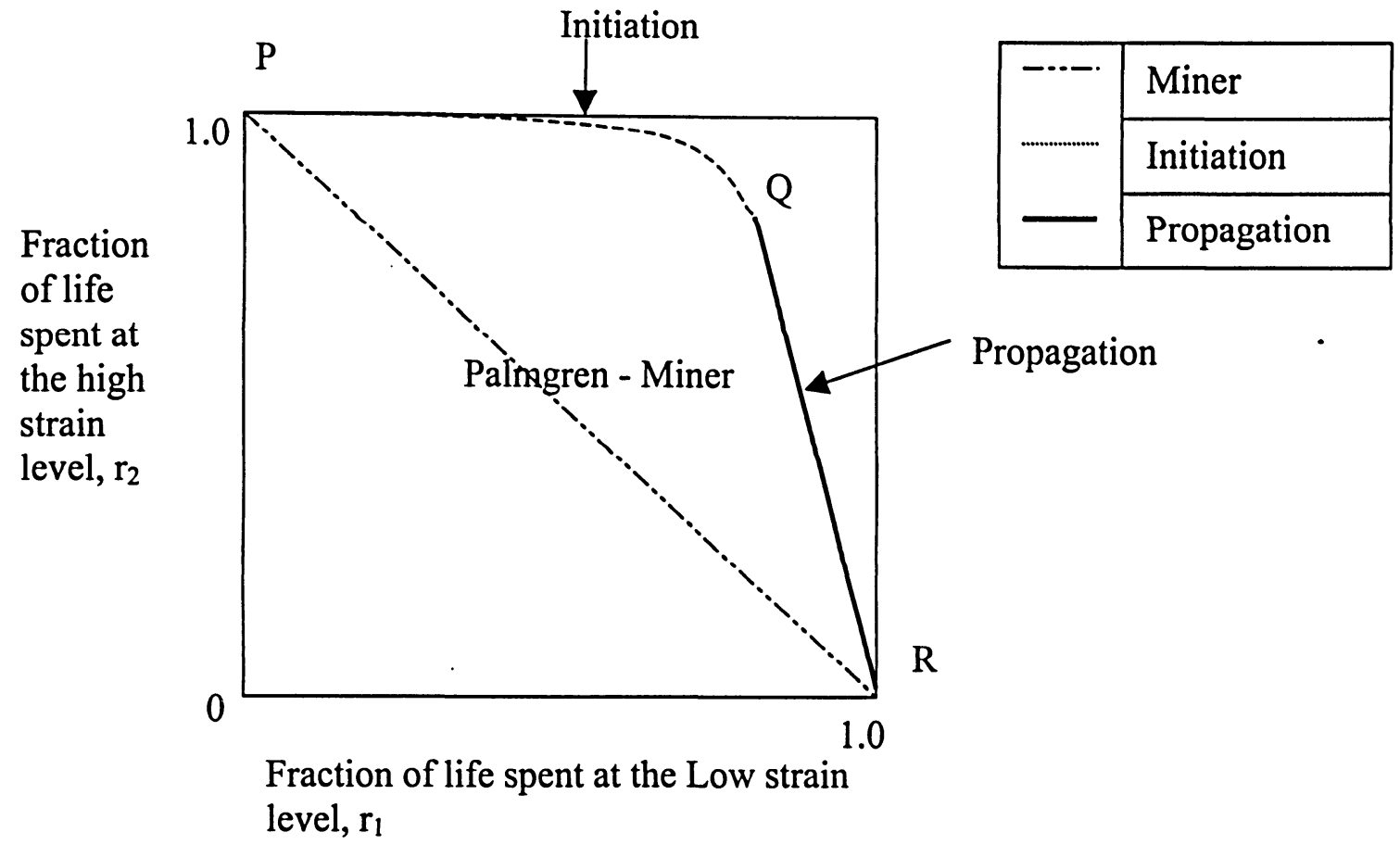

Figure 1.6 Schematic representation of the cumulative damage curve based on the modified Ibrahim-Miller model [22].

where $\phi$ and $\alpha_{1}$ in equation (1.15b) are material constants, and $\left(\Delta \gamma_{p}\right)$ is the plastic shear strain range. From this equation, a linear relationship between the initial cycle ratio, $r_{1}$, and the final ratio, $r_{2}$, in two level cycling can be found for $r_{1}$ in excess of the initiation boundary $\mathrm{r}_{\mathrm{I}, \mathrm{I}}=\mathrm{N}_{\mathrm{I}, \mathrm{l}} / \mathrm{N}_{\mathrm{f}, \mathrm{l}}$. To determine the phase boundary between initiations and stage $\mathrm{I}$ propagation, data from a series of two level strain-controlled tests are then collected and plotted in the $r_{1}-r_{2}$ frame. An example of this type of plot and its comparison with linear rule is shown in Figure 1.6. In further study by Miller and Ibrahim, the damage equation for stage I propagation has been described as:

$$
D=\frac{a}{a_{f}}=\left(\frac{a_{i}}{a_{f}}\right)^{(1-r)\left(1-r_{1}\right)}
$$

Where $a_{i}$ and $a_{f}$ are initial and final crack length; $r$ and $r_{l}$ are initial and final cycle ratio. 


\subsubsection{Energy Based Damage Theories:}

Garud [24] has proposed a new approach to the evaluation of fatigue under complex loading; it relates fatigue life to the plastic work per cycle. The plastic work essentially represents an integrated effect of the two most important quantities generally believed to govern the fatigue process, namely the shear stresses and the plastic strains. The constitutive relations used in his analysis are time-independent and thus the phenomena such as strain-rate sensitivity and creep are excluded. Fatigue life prediction using this approach requires only the uniaxial cyclic stress-strain curve and uniaxial fatigue test results on the smooth specimens. The effect of hydrostatic stress and of mean stress yielding on fatigue life can be included in the analysis when test data are available.

Golos and Ellyin [25] developed a preliminary damage model by using plastic strain energy density as a parameter. Numerous investigations have been carried out to correlate the fatigue life of material with either the strain or stress range. More recently, attempts have been made to relate the fatigue life with plastic strain energy absorbed during a cycle. Ellyin [26] have proposed a special form of the cyclic strain energy density as a damage parameter. This form of the strain energy density combines the plastic strain energy associated with tensile mode, which facilitates crack growth. It is termed the total strain energy density, $\Delta w_{t}$ and is shown in Figure 1.7. This theory has a number of desirable features such as being consistent with the notion both low and high-cycle fatigue regimes, and it is related to the mechanical input energy. Theoretically, plastic strain energy absorbed in a complete cycle can be obtained by integrating the area included in a hysteresis. It is, therefore, also referred to as the hysteresis energy and denoted by $\Delta \mathrm{w}_{\mathrm{p}}$.

It was later found that some inefficiency was associated with plastic strain approach. For example, the effect of mean stress cannot be directly incorporated in the determination of the hysteresis energy. Also, for the low strain-high cycle fatigue, the plastic strain energy density is very small. In some case though the macroscopic (bulk) response of the material is elastic or quasi-elastic, microscopic (local) plastic deformation may still exist in the material due to the non-uniformity of the local strain distribution or due to the 
strain concentration by high pre-straining. To overcome these shortcomings, Golos and Ellyin [27] modified the plastic strain energy-based model by using total strain energy density $\Delta \mathrm{w}^{\mathrm{t}}$. The total strain energy density combines both plastic $\Delta w_{p}$ and elastic $\Delta w_{e}$ portion. The calculation of total energy range $\Delta w_{t}$ is obtained from:

$$
\Delta w_{t}=\Delta w_{e}+\Delta w_{p}
$$

All stress or strain based criteria lack the consideration of the multiaxial stress-strain response of the material as a crucial part of the fatigue process. The fatigue process is generally believed to involve cyclic plastic deformations, which are dependent on the path dependence of the fatigue process sufficiently. The energy concept includes the explicit consideration of the multiaxial stress-strain response. One of the major shortcoming of this approach is, energy is a scalar quantity whereas damage is a vector quantity hence you cannot relate these quantities which is violation of physics.

Lachowicz [28] proposed a method of identification and calculation of components of strain energy density under cyclic and random loading causing elastic-plastic strain in the material. There are some troubles connected with calculation of strain energy density during loading change in the case of random loading by direct integration of the closed and open hysteresis loops.

During schematization of the random loading history, the discrete history of the measured quantity is replaced by set of equivalent range pairs and single ranges. Each distinguished basic or complementary range and each single range is characterized by the amplitude, mean value and time of duration.

Tachankov [29] has developed an incremental approach to estimate the hysteresis energy during the random loading. In his tests, the loading histories included constant amplitude step-up and step-down and random loading with uniformly and Gaussion distribution of stresses. The calculation of dissipated energy under random loading was based on the 
von Mises material behavior. He correlated the dissipated hysteresis energy with the fatigue life of type 35 steel specimens. The failure occurred when a critical boundary value for dissipated energy was reached.

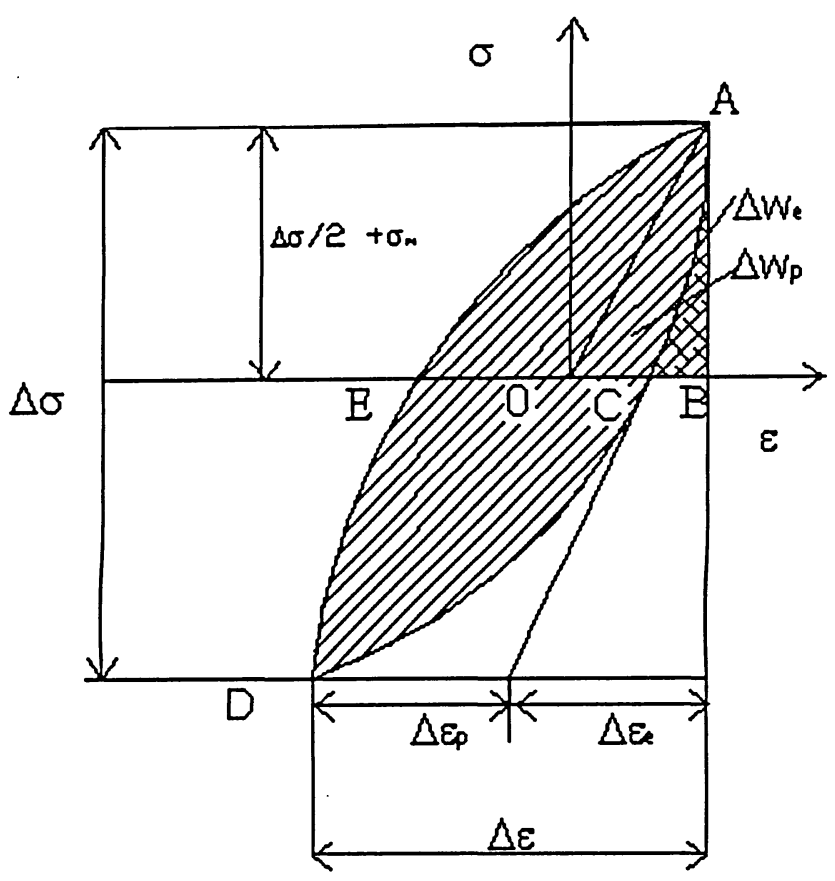

Figure 1.7 Energy Based Approach - Hysteresis Loop [25].

\subsubsection{Continuum Damage Mechanics (CDM) Approaches:}

This approach is developed based on the original concept of Kachanov and Rabotnov [30] in treating creep damage problems. The success of CDM application in modeling the creep damage process has encouraged many researchers to extend this approach to ductile plastic damage, creep-fatigue interaction, brittle fracture and fatigue damage. Chaboche [31] postulated that fatigue damage evolution per cycle could be generalized by a function of the loading condition under completely reversed strain-controlled condition. By measuring the change in tensile load-carrying capacity and using the effective stress concept, the formulated a nonlinear damage evolution equation as:

$$
D=1-\left[1-r^{1\left(1-\alpha_{c}\right)}\right] \frac{1}{\left(1+\beta_{c}\right)}
$$


where $\beta_{c}$ is a material constant, $\alpha_{c}$ is a function of the stress state and $r$ is a cycle ratio. This damage model is highly nonlinear and is able to account for the mean stress effect. It is therefore called a nonlinear continuous fatigue damage model. This model has three main advantages. First, it allows for the growth of damage below the initial fatigue limit. Second, the model is able to take into account the influence of initial hardening effect. Third, mean stress effect is directly incorporated in the model. However, since a scalar damage variable is employed and the model is written in its uniaxial form involving the maximum and mean stresses, difficulties will inevitably be present when the model is extended to multiaxial loading conditions.

The CDM-models mentioned were mainly developed for uniaxial fatigue loading. Some difficulties arise when these models are extended to multiaxial loading. To overcome these difficulties chow and Wei [32] have recently attempted a generalized threedimensional isotropic CDM-model by introducing a damage effect tensor. However, due to complexity of non-proportional multiaxial fatigue problems, a three-dimensional anisotropic CDM model does not yet exist. Though Chaboche great efforts are still needed to obtain an appropriate generalized prediction model for cumulative fatigue damage already proposed the framework.

\subsubsection{Critical plane Damage Theories:}

Fatigue analysis using the concept of critical plane is very effective because the critical plane concept is based upon the fracture mode or the initiation mechanism of cracks. Brown and Miller [33] defined damage parameter as a combination of maximum shear $\operatorname{strain}\left(\gamma_{\max }\right)$ and normal strain $\left(\varepsilon_{n}\right)$ components on the plane of maximum shear strain:

$$
\gamma_{\max }+C \varepsilon_{n}=f\left(N_{f}\right)
$$


Stulen and Cumming [34] defined damage parameter as combination of normal stress $\left(\sigma_{n}\right)$ and maximum shear $\operatorname{stress}\left(\tau_{\max }\right)$ :

$$
\tau_{\max }+C \sigma_{n}=f\left(N_{f}\right)
$$

where $C$ in equation (1.19) and equation (1.20) is a material constant.

Fatemi-Socie [35] came up as combination of maximum strain and maximum normal stress components acting on the critical plane as:

$$
\gamma_{\max }\left[1+\mathrm{n} \cdot \frac{\sigma_{\mathrm{n}}{ }^{\max }}{\sigma_{\mathrm{y}}}\right]=\mathrm{f}\left(\mathrm{N}_{\mathrm{f}}\right)
$$

In equation (1.21), $\left(\sigma_{n}{ }^{\max }\right)$ corresponds to the maximum normal stress acting on the plane of maximum shear strain, $\left(\sigma_{y}\right)$ is the yield strength of material, and constant $n^{*}$ in Fatemi-Socie parameter unifies shear and tensile fatigue data.

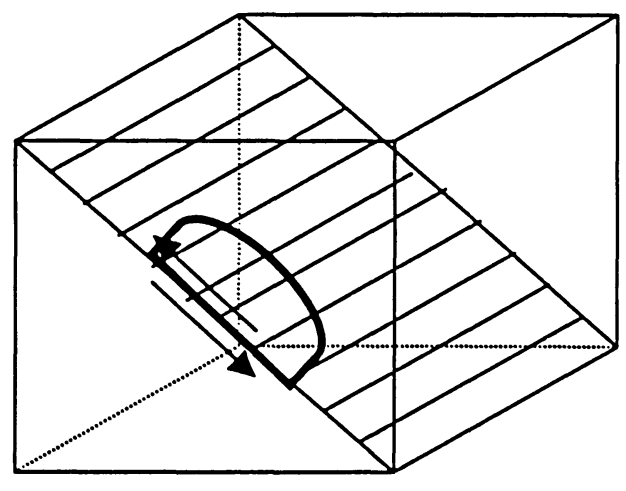

Case A

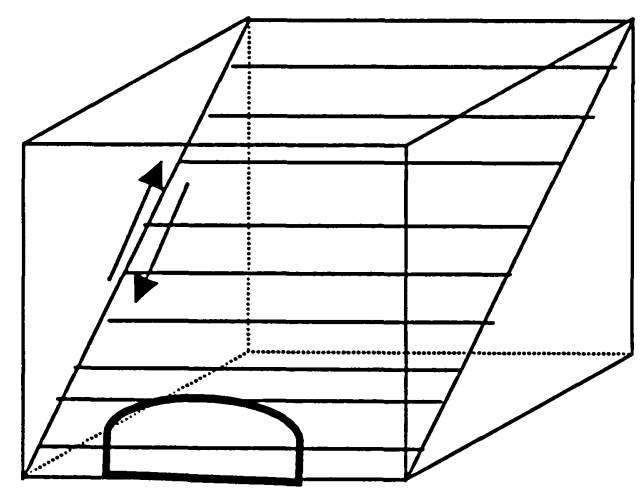

Case B

Figure 1.8 Schematic presentation of different Critical Planes [33] 
The constant is $n^{*}=0.6$ for 1045 steel. Most researchers have shown that shear stress is more dominant factor. The different critical planes are shown in the Figure 1.8 to support above-mentioned theory [33].

\subsubsection{Energy based-critical plane damage approaches:}

Critical plane parameters have been criticized for lack of adherence to rigorous continuum mechanics fundamentals. Some of the below mentioned researchers used the energy criterion in conjunction with the critical plane approach. Energy-critical plane parameters are defined on specific planes and account for states of stress through combinations of the normal and shear strain ranges. These parameters depend upon the choice of the critical plane and the stress and strain ranges acting on that plane. Lagoda, [36] showed a sum of the elastic and plastic strain energy density in the critical plane for description of experimental data obtained from the fatigue test 35NCD16 steel, GGG40 and GG60 cast irons subjected to constant amplitude cyclic tension-compression, torsion and variable-amplitude tension. The parameter of specific work of selected stress on selected strain in the critical plane is an effective parameter, which is used to express the fatigue life of material under a desired loading condition. The critical plane is the plane where the parameter of normal strain energy density reaches its maximum. Lagoda $[37,38]$ also accounted for the stress and stress sign during determination of the energy density allowing one to distinguish the strain energy density at the tension and compression path.

Recently, Varvani [39] has developed a fatigue damage approach. The proposed parameter is sum of the normal energy and shear energy range calculated for critical plane on which the stress and strain Mohr's circles are largest during the loading and unloading part of a cycle. The normal and shear energies used in this parameter are divided by the tensile and shear fatigue properties. The details of this model are discussed in chapter 2 .

The present thesis, will further study Varvani's critical plane-energy based damage parameter including several phenomenological factors, such as load dependence, multiple 
damage stages, nonlinear damage evolution, load sequence and interaction effects, and small amplitude cycle below fatigue limit for smooth component subjected to uniaxial variable amplitude fatigue loading condition. 


\section{CHAPTER TWO}

\section{Variable Amplitude Spectrum and Application}

Fatigue damage is one of the most frequent causes of breakdown in operation. Hence the problem of reliable estimation of fatigue strength of either material or machine parts continues to be essential, not only in the development of new devices but also in the assessment of the fatigue strength of the original construction. Some of the common practical applications of fatigue failure in real life situations are described in this chapter.

\subsection{Aircraft Industry Application:}

One of the typical examples for random fatigue loading is the design of wing loading spectrum in airplanes. Everett [41] has carried out studies on design of spectra for simple block sequence of loads in commercial airplanes. The material used for this study was 2024-T3 aluminum sheet taken from a special stock of material at the NASA Langley research center, which has been used for fatigue and fracture studies over several decades. The alloy 2024 has been used in the lower wing skin of many commercial transport aircraft. The material has yield strength of $52 \mathrm{ksi}$ and an ultimate strength of 72 ksi. The fatigue endurance limit of this material is approximately $18 \mathrm{ksi}$ and the nominal thickness is $0.090 \mathrm{in}$. The study consists of five different flight types for random loading. Flight number one is the most severe and occurs only once in 5000 flights. Flight number two occurs 13 times, flight number three occurs 215 times, and flight number four occurs 1067 times, respectively, in the 5000 cycles sequence. Everett has done this study to account for sequence loading effect in commercial transport. The five loading histories were used to find out the life of component. The loading history was also rearranged in increasing order to evaluate for load sequence effect. 


\subsection{Automobile Industry Application:}

All the moving parts in vehicles are subjected to some kind of fatigue loading. Some of the common examples in automobile industry are suspension load, axle load, bracket vibration which are used by society of automotive engineers. Dowling and Wilison [42] has discussed extensive fatigue testing and analysis program conducted by cumulative damage division of fatigue design and evaluation committee. In this program, two steel specimens, Man-ten and RQC-100 are used. The former is hot-rolled steel having yield strength of about $47 \mathrm{ksi}$, and the latter is rolled, quenched and tempered steel with a yield strength of $120 \mathrm{ksi}$. Tests were conducted using three vehicle service load histories for suspension, axle and bracket vibration. For each test, loads were repeatedly applied, with several load levels being investigated for each combination of material and load history. The fatigue failure applications is not limited to automobile but is applied to the entire transportation industry.

\subsection{Nuclear Power Plant Application:}

To study the behavior of fatigue crack in nuclear power plant the expert organization of Fortnum Nuclear Services Ltd. has given the technical assistance for Loviisa [48]. To understand failure and potential failure mechanism of components is the key issue to collect input data. In order to examine the cracks, ultrasonic and eddy current are used. The result and feedback of measured ultrasonic and eddy current examinations have the key role when assessing the properties of cracked components.

\subsection{Civil Engineering Application:}

Fatigue damage accumulation in steel structures under random loading is a common phenomenon. Agerskov [47] has determined the fatigue life of welded joints by conducting experiments and fracture mechanics analysis. The fatigue tests and the critical plane-energy method have been carried out using load histories, which is realistic in relation to the types of structures studied, i.e. primarily bridges, offshore structures and chimneys. In general, the test series was carried for constant amplitude and variable 
amplitude fatigue. Civil structures such as offshore, highway bridges and chimneys are frequently subjected variable amplitude loading spectra.

\subsubsection{Offshore Structures:}

In the investigations on offshore structures, five different types of load histories have been used. These load histories are generated by computer program and developed at the Department of Structure Engineering and Materials of the Technical University of Denmark [47]. The program simulates a stationary Gaussian stochastic process in real time. Only the extremes of the process are needed, since the load course between consecutive extremes is considered unimportant. In Agerskov's model [47], the next extreme to be generated will be depending only on the present extreme, and not on the preceding load history, i.e. it has a one-step memory. The load histories used in the investigations on the offshore structures are equally in tension and compression and with irregularity factors, I, varying from 0.745 to 0.987 . For narrow band loading, the irregularity factor will be close to unity. Typical load histories for fixed offshore structures will be broader banded, with irregularity factors in the range from $\sim 0.6$ to 0.8 . These are some of the features, which Agerskov's used in study for offshore structures.

\subsubsection{Bridges:}

\subsubsection{Steel bridge Deck:}

For steel bridge deck, fatigue design curves have traditionally been obtained from constant amplitude tests. This is not a realistic in relation to traffic loading, which is the variable loading or random on the steel bridge decks. The main aim of Agerskov's[47] study was to carry out test series and analytical investigations for the difference between the fatigue lives under variable amplitude and constant amplitude loading. The variable amplitude loading that is used in the fatigue tests of this investigation has been determined from the strain gauge measurements on the orthotropic steel deck structure of the Faro Bridge in Denmark. Strain gauge measurements were carried out at 10 different locations in the orthotropic deck. The measurement area is placed at a distance of approximately $8 \mathrm{~m}$ from the simple support of the bridge girder on the nearest bridge pier. The length of the bridge spans was approximately $80 \mathrm{~m}$. This location of the strain gauges 
means that only local bending effect in the deck structure will be registered, whereas the stresses due to global bending in the bridge girder will be negligible. The 10 strain gauges are placed in two sections between two transverse diaphragms. One of these sections is in the middle of the longitudinal stiffener span, which has a length of $4 \mathrm{~m}$, and the other section is placed at a distance of $0.5 \mathrm{~m}$ from one of the transverse diaphragms. Four of the strain gauges in each section are placed on the bottom of trapezoidal longitudinal stiffener of the deck plate. The fifth gage in each section is placed on the butt weld between the trapezoidal stiffener and the deck plate. In this way variable realistic loading data are compared with constant amplitude data used in other early studies [47].

\subsubsection{Concrete Bridge:}

Many concrete structures such as bridges have experienced fatigue cracks under long cyclic loadings caused by trucks etc. The crack strongly affects the normal use and durability of the structure. Analysis reveals that unreasonable fatigue strength and unreasonable design rule are main reasons [48]. Nowadays, because of the limitation of experimental methods, structures are wholly designed by uniaxial fatigue strength or uniaxial compression fatigue strength under constant amplitude loading. Practice proved that the result is conservative or dangerous. Song [48] has carried out some experiments on uniaxial tensile strength and uniaxial compression strength of plain concrete under constant amplitude cyclic loading. Studies in this area have been carried out by other researches on biaxial compression strength under constant amplitude or variableamplitude loading, but the experiment and analysis on biaxial tension-compression strength have not been done yet. Analysis proved that Miner's Rule is not wholly suitable to the fatigue crack of concrete material. So there is need for more suitable model, which works for uniaxial and biaxial fatigue loading condition.

\subsubsection{Chimneys:}

The variable amplitude loading that was used in the investigation of a chimney has been determined from wind tunnel tests. An undamped chimney model, subject to transverse oscillations was studied [47]. Two load histories were used, both determined from the 
strain gauge measurements on the model. These load histories are equal in tension and compression and narrow-banded, with an irregularity factor of $I=0.998$.

\subsubsection{Pile Supported Structures:}

It is common that piles and pile-supported structures are subjected to cyclic loading. For example, offshore structure (such as ocean petroleum platform) with pile foundation subjected is to cyclic wave loading. Many researchers [48] have worked to accurately predict static and dynamic soil-pile-superstructure response through various numerical analyses. Only the static/constant loading tests are carried out because of a limited series of field and complexity of tests. For piles of ocean petroleum platform, another problem is seawater-pile interaction. The Seawater-pile interaction is commonly considered using water-damping method. It goes under continues cyclic loading due to waves in seawater.

\subsubsection{Ice and Offshore Structures:}

The moving ice induces offshore structures strong vibrations. In order to evaluate fatigue life of the structure under ice force, it necessary to estimate the distribution of ice thickness, ice velocity as well as impacting frequency at least one year. This needs sea ice observation and simulation in long term. Systematic sea ice observations have been conducted in Bohai Bay of China and large amount of data has been accumulated. Short term ice simulation in small-scale has been carried. Some calculation parameters and the viscous-plastic constitutive laws of the ice are modified [48]. 


\section{CHAPTER THREE}

\section{Fatigue Damage Analysis and Modeling}

\subsection{Introduction:}

Reliable estimate of fatigue life at every stage in the construction of a structure is necessary because it is operationally difficult, uneconomic and time-consuming to test each component or the entire structure experimentally in the laboratory or directly during operation. The results of useful life estimation are not always satisfactory. This is due to the fact that none hypothesis is sufficiently general to consider the effects of all the interacting factors in fatigue life evaluation. Most service loading histories have variable amplitudes and can be quite complex. Several methods have been developed to deal with variable amplitude loading using the baseline data generated from constant amplitude tests.

The total fatigue life of a component may be divided into two different regimes: crack initiation and crack propagation. A general mechanism for crack initiation is based on coarse slip processes. Coarse slip occurs on the favorably oriented crystallographic planes with single grains of material. Cyclic loading causes reversed slip and the formation of persistent slip bands. Materials displaced in local regions forming intrusion, extrusions and ultimately de-cohesion occurs, forming a microcrack. The applied shear stress and resulting shear strains are the dominant parameters for this initiation process. The definition of the crack initiation is usually taken as the formation of an "engineering size" crack (usually between 0.1 to $5 \mu \mathrm{m}$ in surface length). The analysis developed in this chapter deals with fatigue damage assessment of engineering component subjected to constant and variable amplitude uniaxial loading conditions based on Varvani's energy based-critical plane approach [39]. According to this approach, the proposed parameter is 
sum of the normal energy and shear energy range calculated for critical plane on which the stress and strain Mohr's circles are largest during the loading and unloading part of a cycle. The normal and shear energies used in this parameter are divided by the tensile and shear fatigue properties.

\subsection{Elements of Fatigue Damage Assessment based on Varvani's Approach [38]:}

Figure 3.1 presents a thin walled tubular specimen subjected to combined axial and torsional fatigue. The tensorial stress and strain components for an infinitesimal element on the tube can be presented by:

$$
\begin{aligned}
& \Delta \varepsilon_{i j}=\left(\begin{array}{ccc}
-v_{e f f} \Delta \varepsilon_{a p} & \Delta\left(\frac{\gamma_{a p}}{2}\right) & 0 \\
\Delta\left(\frac{\gamma_{a p}}{2}\right) & \Delta \varepsilon_{a p} & 0 \\
0 & 0 & -v_{e f f} \Delta \varepsilon_{a p}
\end{array}\right) \\
& \Delta \sigma_{i j}=\left(\begin{array}{ccc}
0 & \Delta \tau_{a} & 0 \\
\Delta \tau_{a} & \Delta \sigma_{a} & 0 \\
0 & 0 & 0
\end{array}\right)
\end{aligned}
$$

where axial and shear strain ranges $\Delta \varepsilon_{n}$ and $\Delta\left(\frac{\gamma_{\max }}{2}\right)$ respectively are given by the following equations:

$$
\begin{aligned}
& \Delta \varepsilon_{a p}=\Delta \varepsilon_{a} \sin \theta \\
& \Delta\left(\frac{\gamma_{a p}}{2}\right)=\Delta\left(\frac{\gamma_{a}}{2}\right) \sin (\theta-\phi)
\end{aligned}
$$


where $\varepsilon_{a p}$ and $\frac{\gamma_{a p}}{2}$ are the applied axial and shear amplitude strains, respectively. The angle $\theta$ is the angle during a cycle of straining at which the Mohr's circle is the largest and has the maximum value of shear strain. Angle $\phi$ corresponds to the phase delay between strains on the axial and torsional axes. In equation (3.2) $\Delta \sigma_{a}$ and $\Delta \tau_{a}$ are the ranges of axial and shear stresses, respectively.

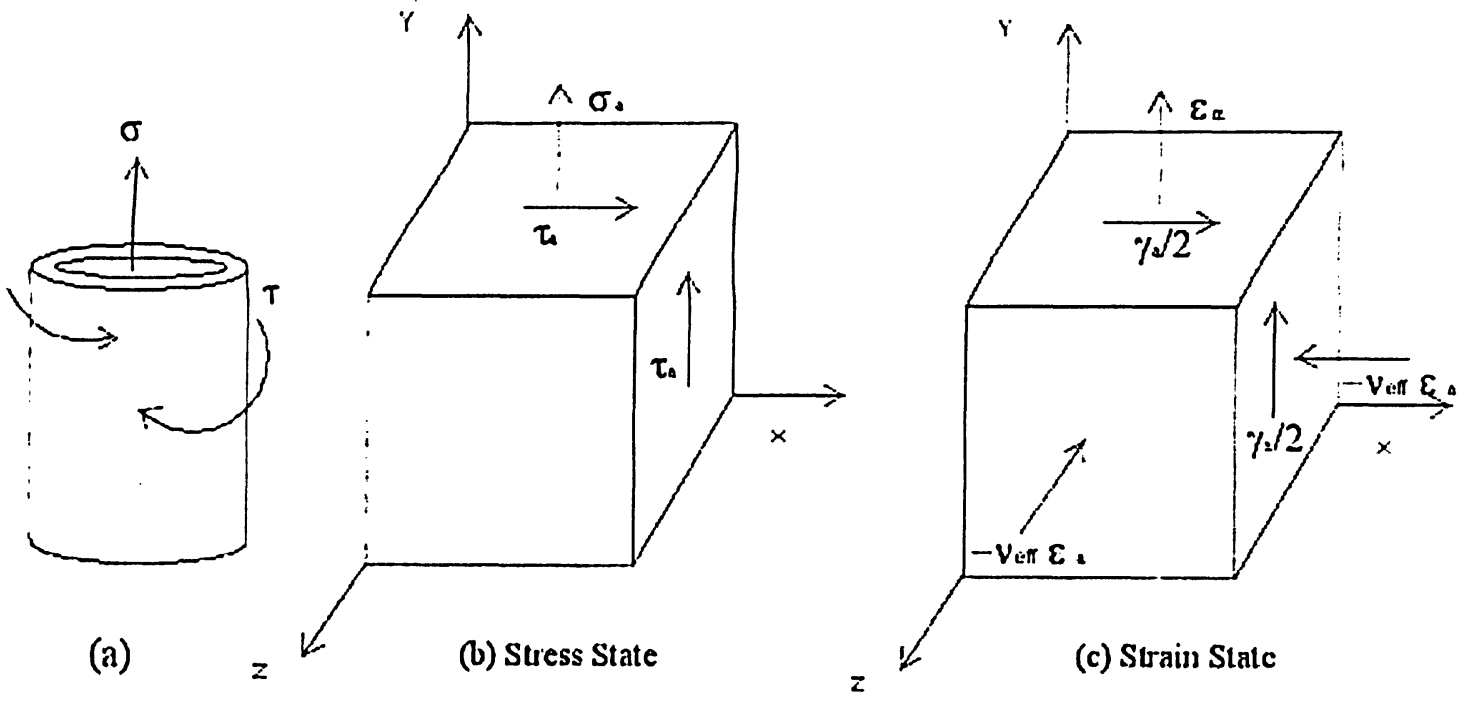

Figure 3.1 3D presentation of stress and strain state for multiaxial loading [38].

Principal strain values $\varepsilon_{1}, \varepsilon_{2}, \varepsilon_{3}\left(\varepsilon_{1}>\varepsilon_{2}>\varepsilon_{3}\right)$ are given as:

$$
\begin{aligned}
& \varepsilon_{1}=\left(1-v_{e f f}\right) \frac{\varepsilon_{a p p}}{2}+1 / 2\left[\varepsilon_{a p}{ }^{2}\left(1+v_{e f f}\right)^{2}+\left(\frac{\gamma_{a p}}{2}\right)^{2}\right]^{1 / 2} \\
& \varepsilon_{2}=-v_{e f f} \varepsilon_{a p} \\
& \varepsilon_{3}=\left(1-v_{e f f}\right) \frac{\varepsilon_{a p p}}{2}-1 / 2\left[\varepsilon_{a p}{ }^{2}\left(1+v_{e f f}\right)^{2}+\left(\frac{\gamma_{a p}}{2}\right)^{2}\right]^{1 / 2}
\end{aligned}
$$


Where $\varepsilon_{a p}$ is applied axial strain, $\gamma_{a p}$ is applied shear strain and $v_{e f}$ is the effective Poisson's ratio, which is given by:

$$
v_{e f f}=\frac{v_{e} \varepsilon_{e}+v_{p} \varepsilon_{p}}{\varepsilon_{e}+\varepsilon_{p}}
$$

Where $v_{e}=0.3$ is the elastic Poisson's ratio and $v_{p}=0.5$ is the plastic Poisson's ratio. The unknown axial elastic and plastic strain are calculated, respectively:

$$
\begin{aligned}
& \varepsilon_{e}=\frac{\sigma_{a}}{E} \\
& \varepsilon_{p}=\varepsilon_{a y}-\frac{\sigma_{a}}{E}
\end{aligned}
$$

Where $\varepsilon_{e}$ is axial elastic strain, $\varepsilon_{p}$ is axial plastic strain, $\varepsilon_{a y}$ is axial total strain along $\mathrm{Y}$ axis, $\mathrm{E}$ is Modulus of elasticity, $\sigma_{a}$ is axial total stress. The range of maximum shear strain $\Delta\left(\frac{\gamma_{\max }}{2}\right)$ and the corresponding normal strain range $\left(\Delta \varepsilon_{n}\right)$ on the critical plane at which both strain and stress Mohr's circles are the largest during loading (at the angle $\theta_{1}$ ) and unloading (at the angle $\theta_{2}$ ) of a cycle (see Figure 3.2) are calculate as:

$$
\Delta\left(\frac{\gamma_{\max }}{2}\right)=\left(\frac{\varepsilon_{1}-\varepsilon_{3}}{2}\right)_{\theta_{1}}-\left(\frac{\varepsilon_{1}-\varepsilon_{3}}{2}\right)_{\theta 2}
$$




$$
\Delta\left(\varepsilon_{n}\right)=\left(\frac{\varepsilon_{1}+\varepsilon_{3}}{2}\right)_{\theta_{1}}-\left(\frac{\varepsilon_{1}+\varepsilon_{3}}{2}\right)_{\theta 2}
$$

Similarly, the range of maximum shear stress $\left(\Delta \tau_{\max }\right)$ and the corresponding normal stress range $\left(\Delta \sigma_{n}\right)$ are calculated from the largest stress Mohr's circle during loading (at the angle $\theta_{1}$ ) and unloading (at the angle $\theta_{2}$ ) of a cycle are calculate as shown in Figure 3.2:

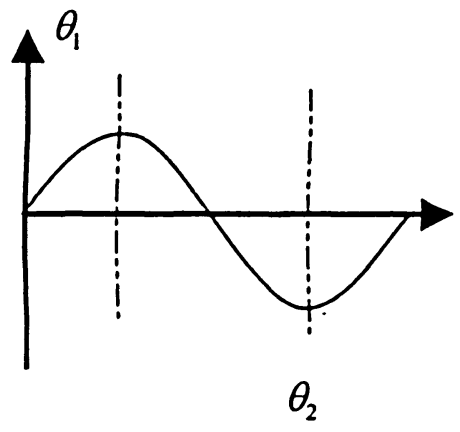

(a) Strain History

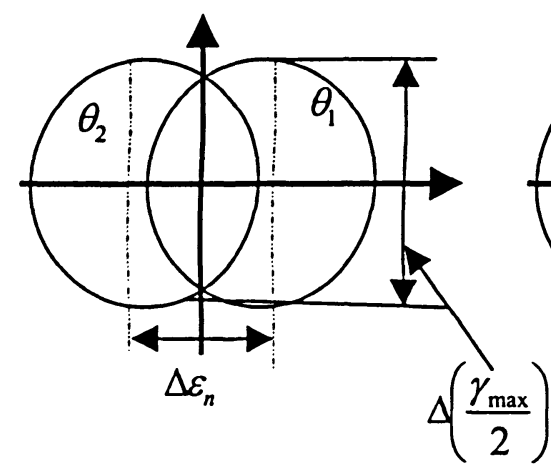

(b) Strain Mohr's Circle

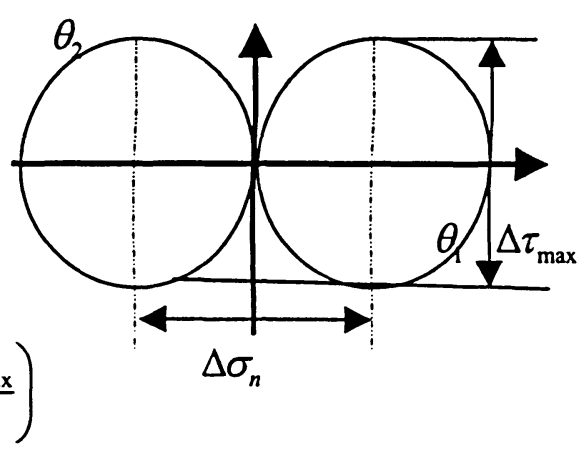

(c) Stress Mohr's Circle

Figure 3.2 Strain history, strain Mohr's circle, and stress Mohr's circle for uniaxial loading condition [38].

$$
\begin{aligned}
& \Delta \tau_{\max }=\left[\frac{\sigma_{1}-\sigma_{3}}{2}\right]_{\theta 1}-\left[\frac{\sigma_{1}-\sigma_{3}}{2}\right]_{\theta 2} \\
& \Delta \sigma_{n}=\left[\frac{\sigma_{1}+\sigma_{3}}{2}\right]_{\theta 1}-\left[\frac{\sigma_{1}+\sigma_{3}}{2}\right]_{\theta 2}
\end{aligned}
$$

where $\sigma_{1}, \sigma_{2}, \sigma_{3}$ are the principal stress values $\left(\sigma_{1}>\sigma_{2}>\sigma_{3}\right)$ and are calculated as: 


$$
\begin{aligned}
& \sigma_{1}=\frac{\sigma_{a}}{2}+1 / 2\left[\sigma_{a}^{2}+4 \tau_{a}^{2}\right]^{1 / 2} \\
& \sigma_{2}=0 \quad \text { (Plane stress condition) } \\
& \sigma_{3}=\frac{\sigma_{a}}{2}-1 / 2\left[\sigma_{a}^{2}+4 \tau_{a}^{2}\right]^{1 / 2}
\end{aligned}
$$

The range of maximum shear stress $\Delta \tau_{\max }$ and shear $\operatorname{strain} \Delta\left(\frac{\gamma_{\max }}{2}\right)$ obtained from the largest stress and strain Mohr's circles during the loading and unloading parts of a cycle and the corresponding normal stress range $\Delta \sigma_{n}$ and normal strain range $\Delta \varepsilon_{n}$ on that plane are the components of the Varvani's approach. In this approach both the normal and shear strain energies were weighted by the axial and shear fatigue properties, respectively.

$$
\frac{1}{\sigma_{f}^{\prime} \varepsilon_{f}^{\prime}} \Delta \sigma_{n} \Delta \varepsilon_{n}+\frac{1}{\tau_{f}^{\prime} \gamma_{f}^{\prime}} \Delta \tau_{\max } \Delta\left(\frac{\gamma_{\max }}{2}\right)=f\left(N_{f}\right)
$$

Considering the effect of axial mean stress, a mean stress correction factor of the form $\left(1+\sigma_{n}{ }^{m} / \sigma_{f}^{\prime}\right)$ in equation (3.19) was employed, and the damage equation with mean stress effect yielded as:

$$
\frac{1}{\sigma_{f}^{\prime} \varepsilon_{f}^{\prime}} \Delta \sigma_{n} \Delta \varepsilon_{n}+\frac{\left(1+\frac{\sigma_{n}^{m}}{\sigma_{f}^{\prime}}\right)}{\tau_{f}^{\prime} \gamma_{f}^{\prime}} \Delta \tau_{\max } \Delta\left(\frac{\gamma_{\max }}{2}\right)=f\left(N_{f}\right)
$$

Where the normal mean stress $\sigma_{n}{ }^{m}$ acting on the critical plane is given by:

$$
\sigma_{n}^{m}=\frac{1}{2}\left(\sigma_{n}^{\max }+\sigma_{n}^{\min }\right)
$$


In equation (3.20), $\sigma_{n}{ }^{\max }$ and $\sigma_{n}{ }^{\text {min }}$ are the maximum and minimum normal stresses which are calculated from the stress Mohr's circles. In fatigue damage assessment under variable amplitude loading condition, the effects of following phenomenological factors are studied:

\subsection{Sequence loading.}

3.4 Memory effect.

3.5 Small cycles.

A schematic view of the model and damage analysis is shown in Figure 3.3, which shows the detail steps involved in the life prediction presented in this study.

\subsection{Effect of sequence loading:}

\subsubsection{Background:}

The order in which cycles are applied has a significant effect in random loading. Due to sequence effect these strain-time histories will yield a very different stress-strain response. According to Kliman [40] under a given amplitude frequency in a loading block, the effect of the loading history can be seen in the different values of the energy to fracture and its dependence on the arrangement of the amplitude. This means that the arrangement of amplitudes in the loading block will significantly influence the energy for blocks with a large number of amplitudes and levels. Golos and Ellyin [25] examined the cumulative damage and the effect of loading sequence. Tests were performed with two, three and four stage loading. A schematic diagram of block loading is as shown in the Figure 3.4. 


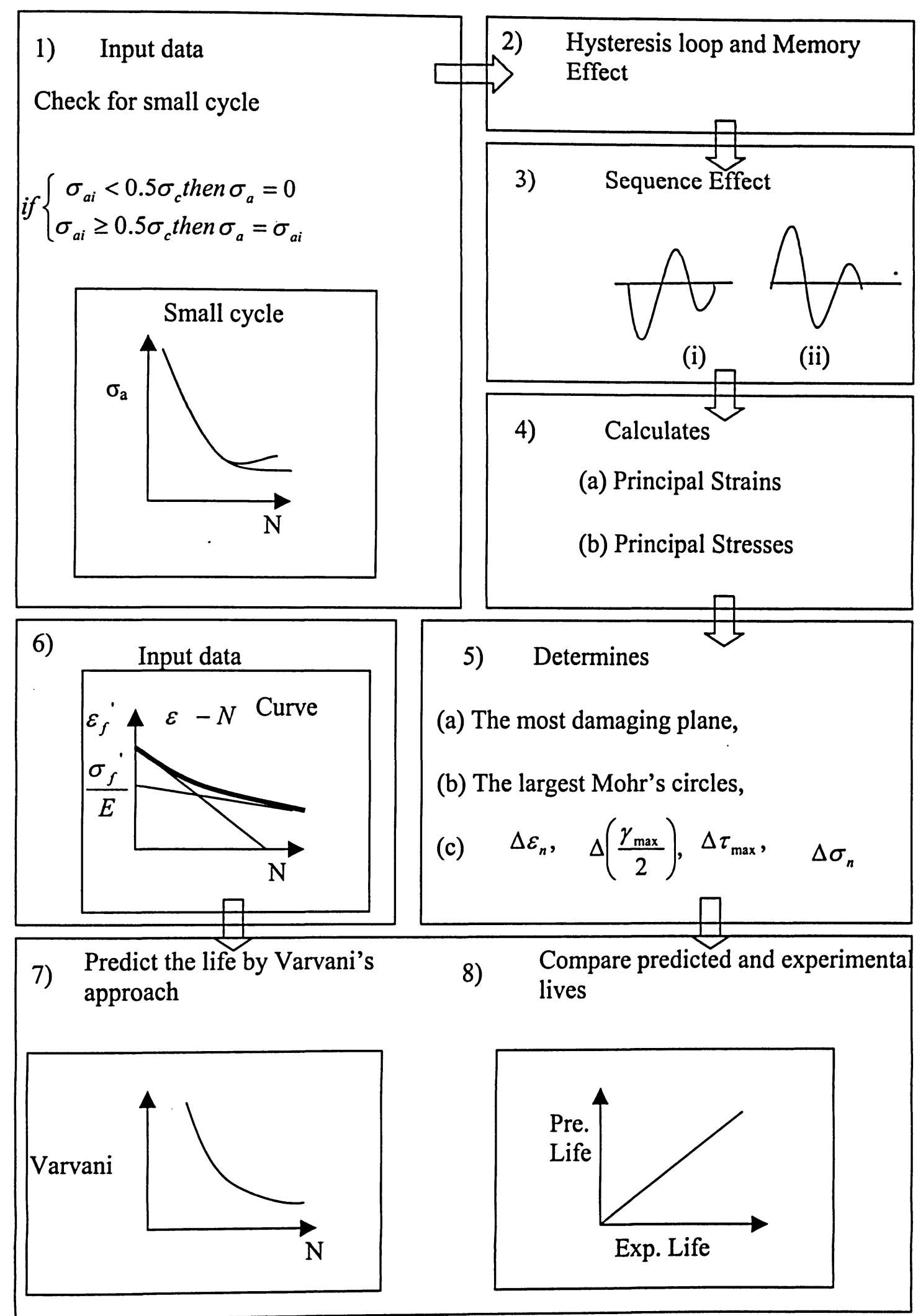

Figure 3.3 Steps of fatigue damage analysis. 


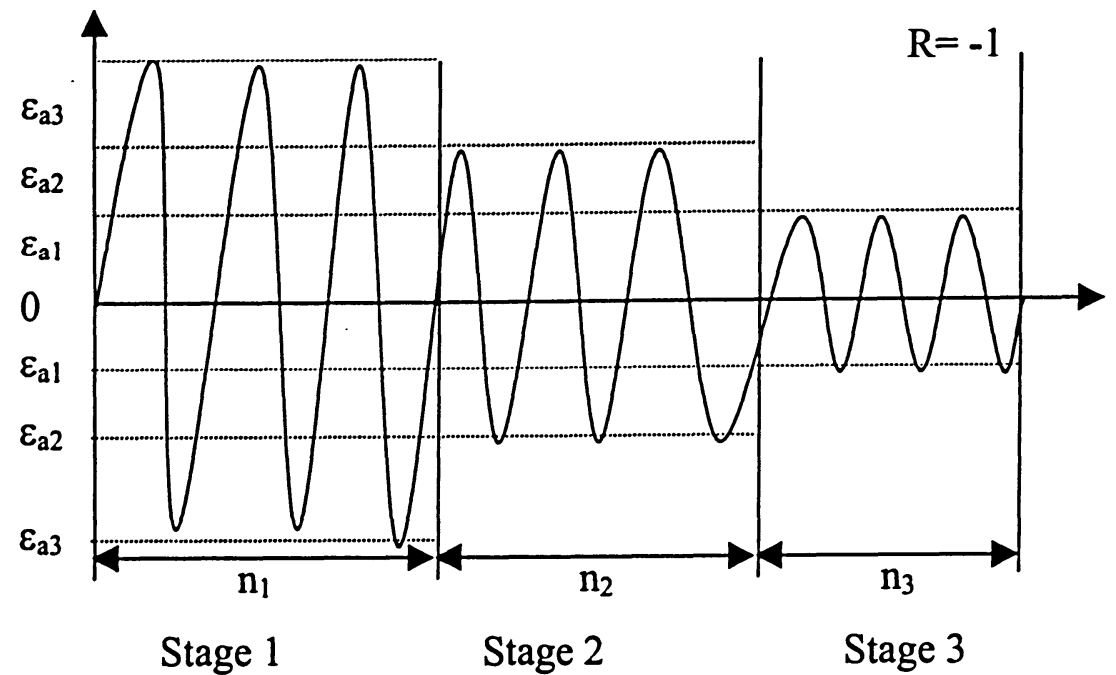

Figure 3.4 A schematic diagram of block/step loading condition [25].

These tests show that changing the loading sequence significantly influences the energies obtained from different loading stages. Hence loading sequence has a significant relationship with damage magnitude.

Everett [41] has done studies to evaluate the loading sequence effects that could exist in commercial fixed-wing fatigue loading spectra. To evaluate this effect, a typical commercial wing spectrum was re-arranged from the smallest load range until the largest load range. Tests on open hole test specimens made of 2024-T3 aluminum alloys were conducted on the normal sequence loading as well as on re-arranged loading. sequence. The test results showed no significant difference between the fatigue lives of normal loading sequence and rearranged loading sequence.

\subsubsection{Description:}

The above discussed sequence effect can be explained with the help of Figure 3.5. The Case 1, Figure 3.5a starts with initial tensile overload followed by a compressive overload and then normal loading condition. The Case 2, Figure $3.5 \mathrm{~b}$ consists of initial compressive overload followed by a tensile overload and then normal loading condition. 
If Cases 1 and 2 are compared, then it is noticed that the only difference between Figures 3.5(a) and (b) is sequence of stresses applied. In Case 1, the hysteresis loops start with stress value $540 \mathrm{MPa}$ and then followed by another positive stress value as $340 \mathrm{MPa}$, but in Case 2 loop start with stress value $340 \mathrm{MPa}$ and followed by another positive stress value of $540 \mathrm{MPa}$. Therefore, in both cases the total stress value remains same and according to Miner's rule as discussed in section 1.1, the strain response should be the same for both above cases. However, when actual strain response is checked, it is found that strains are entirely different.

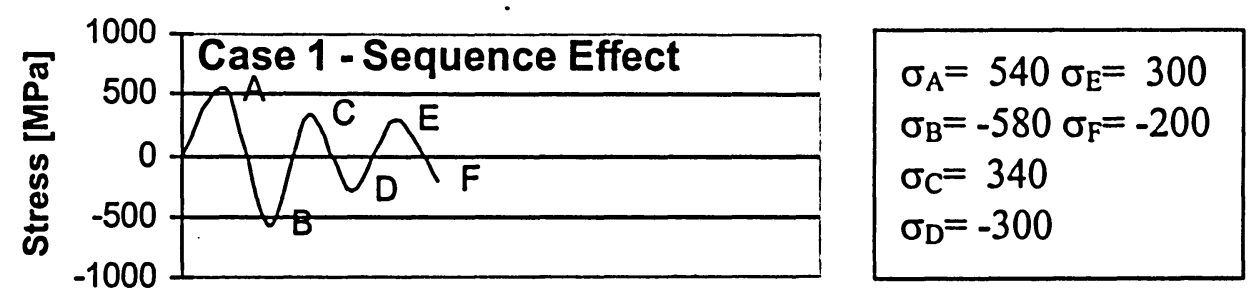

Time

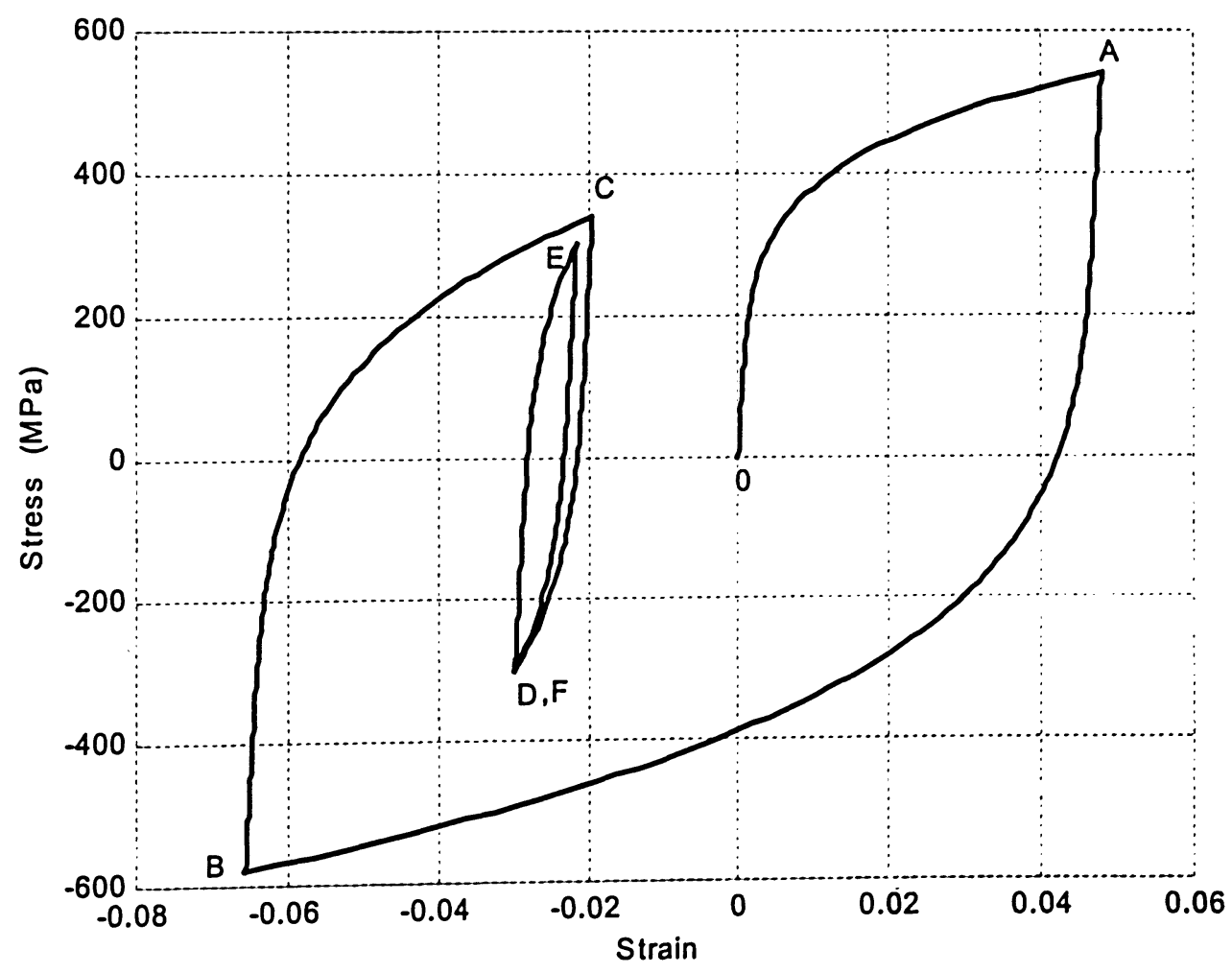

(a) Case 1 


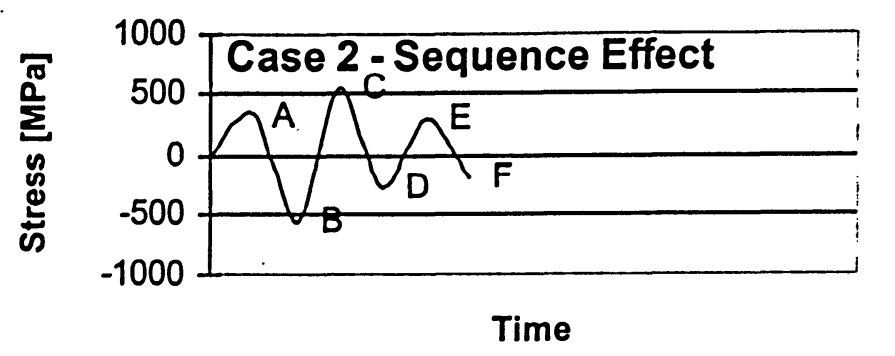

$$
\begin{aligned}
& \sigma_{A}=340 \sigma_{E}=300 \\
& \sigma_{B}=-580 \sigma_{F}=-200 \\
& \sigma_{C}=540 \\
& \sigma_{D}=-300
\end{aligned}
$$

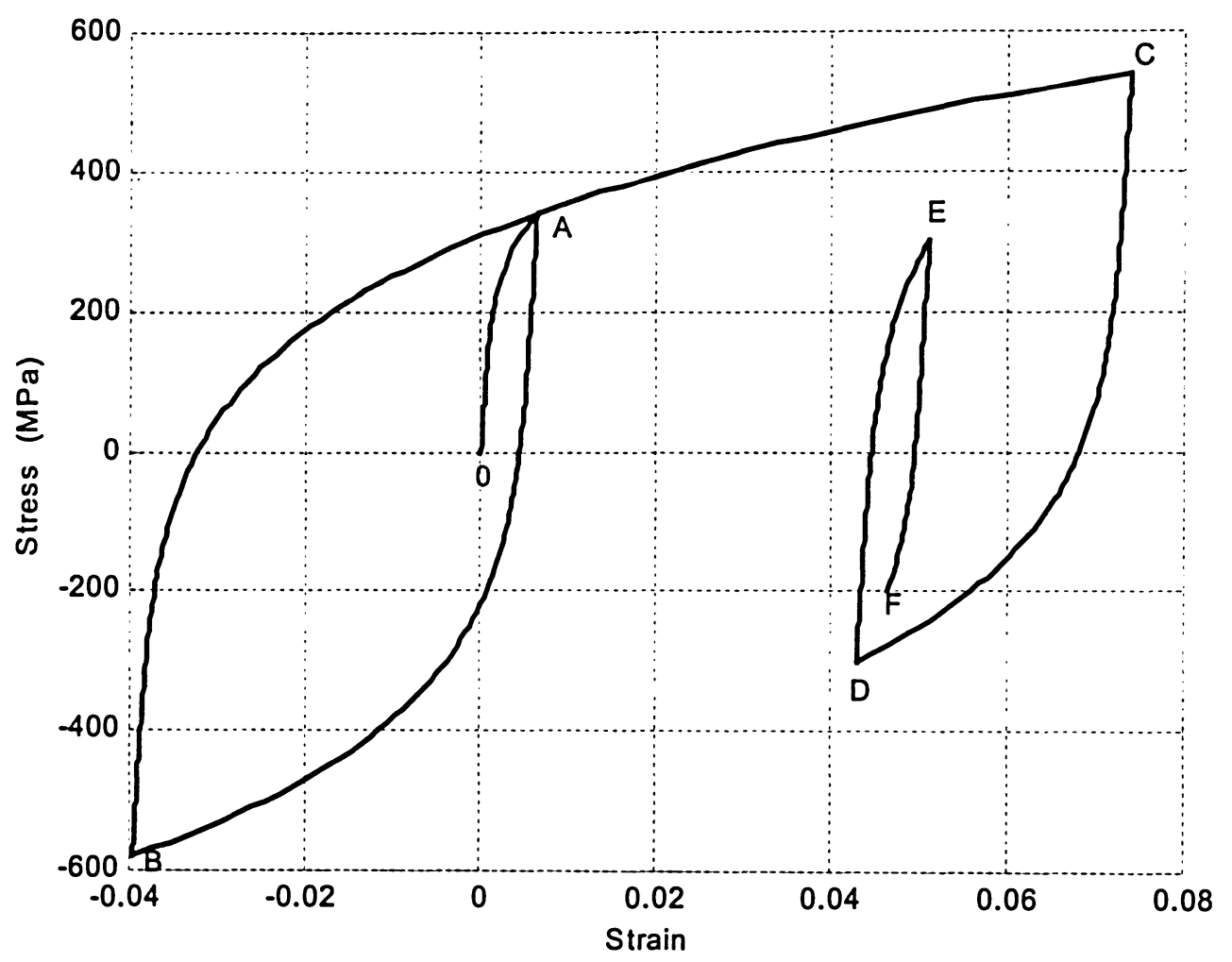

(b) Case 2

Figure 3.5 Schematic Representation of Sequence Effect.

For Case 1 strain value at $D$ is -0.01 and for Case 2 strain value at $D$ is 0.0125 . This shows that sequence effect studied above results in different material damage and deformation, which has to be considered for variable amplitude loading conditions. 


\subsubsection{Implementation:}

In order to incorporate above effect, the equations (3.21 - 3.23) are used. Referring to Figure 3.5, the first 0-A portion of curve is developed by general Ramberg-Osgood equation (3.21) with instantaneous stress values $\sigma_{i}$, strain for $0-\mathrm{A}$ is calculated with Ramberg-Osgood equation as:

$$
\varepsilon_{1} o r \varepsilon_{A}=\left(\frac{\sigma_{i}}{E}\right)+\left(\frac{\sigma_{i}}{K}\right)^{1 / n_{1}}
$$

Where $E$ is Modulus of elasticity, $K$ is cyclic strength coefficient, and $n_{l}$ is cyclic hardening exponent. From all the possible strain values maximum strain value obtained from the equation (3.21) is used to calculate principal strains for 0 -A portion of curve Principal strains and principal stress, effective Poisson's ratio, elastic strain and plastic strain are calculated using maximum strain value obtained from equation (3.21). The previously calculated maximum strain $\varepsilon_{1}$ at point $A$ is then used in equation (3.22) to calculate minimum strain for A-B portion, the principal strain and principal stress are calculated for A-B portion of the hysteresis loops. Once two set of principal stresses and strains are available then maximum shear strain range and normal strain range are calculated from these principal strains and principal stresses. After calculating normal and shear ranges the damage is calculated and stored in one variable. Strain $\varepsilon_{2}$ is calculated for unloading part of cycle (A-B) using Ramberg-Osgood equation as:

$$
\varepsilon_{2} o r \varepsilon_{B}=\varepsilon_{A}-\left[\left(\sigma_{A}-\frac{\sigma_{i}}{E}\right)-2\left(\sigma_{A}-\frac{\sigma_{i}}{2 k}\right)^{1 / n}\right]
$$

The B-C portion of curve (reloading part) is developed by increasing Ramberg-Osgood equation (3.23), which is developed in the similar manner like 0 -A portion except it uses previously calculated minimum strain $\varepsilon_{2}$ (3.22) as the starting point. Then plastic strain, elastic strain, passions ratio, principal strain and principal stress are accordingly 
calculated. For reloading portion of cyclic stress-strain loop (B-C portion), $\varepsilon_{3}$ is calculated using Ramberg-Osgood equation as:

$$
\varepsilon_{3} \circ r \varepsilon_{c}=\left[\left(-\sigma_{B}+\frac{\sigma_{i}}{E}\right)+2\left(-\sigma_{B}+\frac{\sigma_{i}}{2 k}\right)^{1 / n}\right]+\varepsilon_{B}
$$

Where, $\varepsilon_{B}$ is minimum strain at point $\mathrm{B}, \sigma_{B}$ is minimum stress at point $\mathrm{B}$. The fourth $\mathrm{C}$ $D$ portion of curve is calculated using Ramberg-Osgood equation and follows the same unloading Ramberg-Osgood equation as calculated for A-B portion. This chain of loading (equation (3.22)) and reloading (equation (3.23)) will continue till the failure of component occurs. Thus, the sequence effect is incorporated in model through the latest value of strain calculated from equations (3.21 -3.23), and thereafter using them in the next equation. The above mentioned equation also takes care of overload and underload histories because sequence effect uses the last damage quantity in the calculation.

In same regards Macha [36] has introduced sign effect, which is supportive to the above discussion. It is noticed that the negative and positive signs are provided in the bracket, in such a way that the values will always remain positive inside the bracket. In case of loading, the value will be added and in case of unloading, the value will be deducted. The negative value outside the bracket takes care of negative sign (during the unloading cycle). Hence, in sequence effect unloading and reloading (Ramberg-Osgood) equations, the sign effect proposed by Macha was already incorporated as a built-in feature.

\subsection{Memory Effect:}

\subsubsection{Background:}

Dowling and Wilson [42] characterized the memory effect by two rules:

(a) According to Figure 3.6, when the strain next reaches a value ( $D^{\prime}$ in Figure 3.6) at which the direction of straining was previously reversed, a stress - strain hysteresis loop 
is closed, and the stress- strain path beyond this point is the same as if the direction of straining had not been removed.

(b) Once a strain excursion forms a closed loop, this excursion does not affect the subsequent behavior. The above two rules for the memory effect may be used to estimate the entire stress-strain response for any repeating strain history. The repeating history should be considered to begin and end at most extreme strain peak in either directions. The co-ordinates of this most extreme and first peak may then be estimated by assuming that from zero stress and strain the stress- strain path follows the cyclic stress-strain curve.

\subsubsection{Description:}

The above stated rule can be explained by figure 3.6. Looking at this figure it is noticed that an initially maximum stress value of $600 \mathrm{MPa}$ is attained by the hysteresis loop at point $A$, then minimum stress value of $-600 \mathrm{MPa}$ at point $B$, again a maximum value of $600 \mathrm{MPa}$ at point $\mathrm{C}$. However at point $\mathrm{D}$ stress value goes up to $-300 \mathrm{MPa}$ and raises to a positive stress value of $300 \mathrm{MPa}$, thereafter it goes to minimum value of -600 . As it can be seen, DED' forms a small close loop inside a big loop, and once the small loop is constructed it follows the bigger loop path as if the direction of straining was never changed. If the hysteresis loop is plotted according to the sequence effect, the latest stress/strain value will not form a closed loop. Hence, memory effect concept has been carefully monitored and programmed to correspond to the closed hyestersis loop.

\subsubsection{Implementation:}

The above mentioned memory concept is used to develop the close hysteresis loop for random and variable loading. In order to close the loop, the developed computer program should be capable of knowing the limits (i.e. maximum and minimum values are stored from the input data), and these maximum and minimum values are used as restricted boundaries for the loop generated by interpolating $\sigma_{i}$ values in equations (3.21-3.23), which gives respective $\varepsilon_{i}$ values. As the above-mentioned sequence effect is implemented in the program the values generated are not strain controlled. Therefore, Memory effect 
concept has been carefully monitored and programmed to correspond to the closed hysteresis loop in each block loading history.
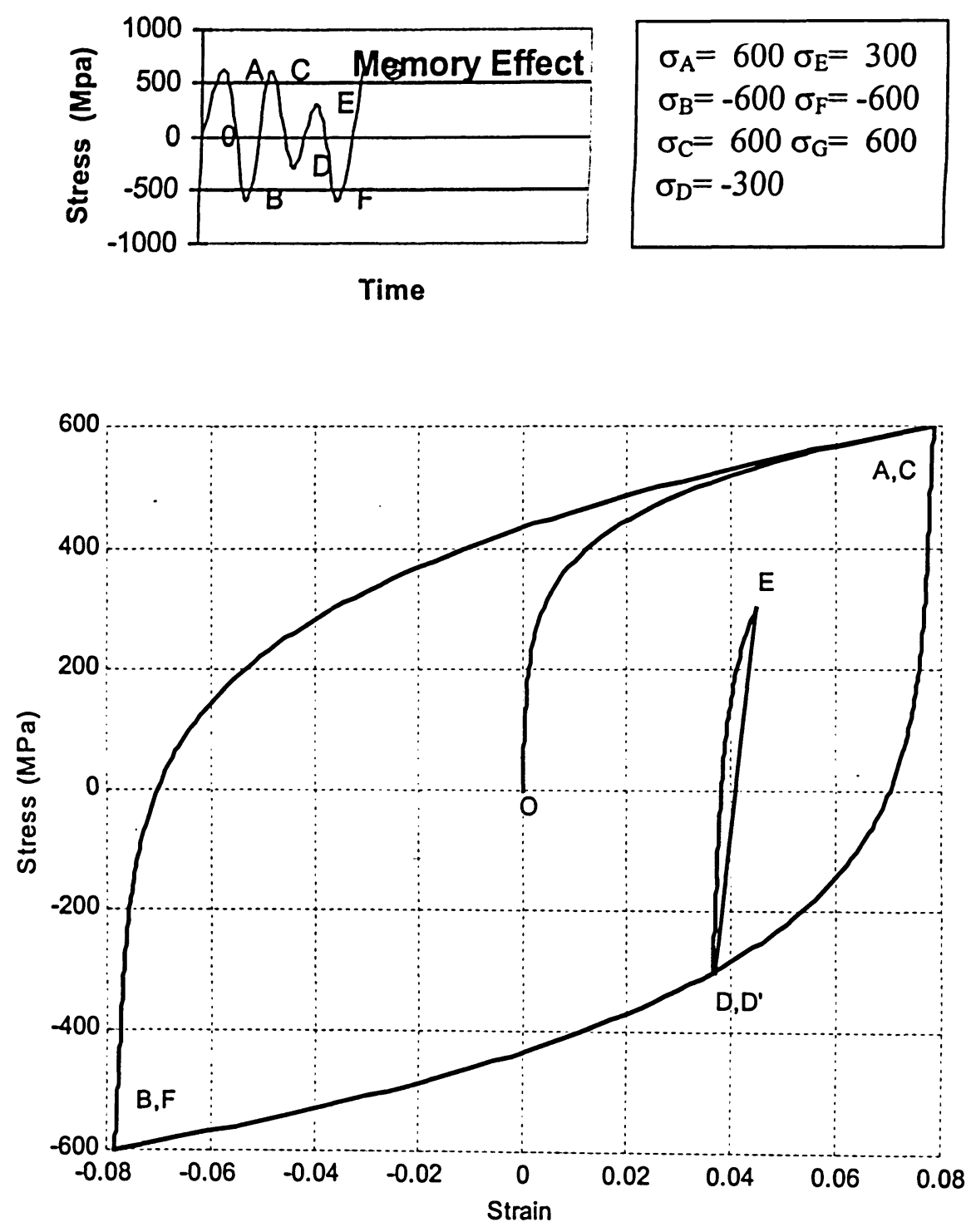

Figure 3.6 Schematic Representation of Memory Effect. 


\subsection{Effect due to small cycles below endurance limit:}

\subsubsection{Background:}

The Palgrim-Miner hypothesis does not take into account the effect of amplitude below the fatigue limit. The suggested hypothesis is valid provided; the value of maximum stress amplitude $\left(\sigma_{\mathrm{a}}\right)$ is not below the fatigue limit $\left(\sigma_{\mathrm{c}}\right)$. Kilman [40] calculated the effect of amplitude below the fatigue limit and above the fatigue limit. At high stresses (where there are no instantaneous stress amplitude $\sigma_{a i}$ less than $\sigma_{c}$ in the loading block) the curve are identical. As the stress decreases (i.e. with an increasing number of amplitude $\sigma_{a i}$ less than $\sigma_{\mathrm{c}}$ in the loading block), the inclusion of the $\sigma_{\mathrm{ai}}$ amplitude in the calculation leads to shorter lives. Pompetzki and Topper [43] conducted extensive studies on overload (OL) and underload (UL) fatigue loading. They demonstrated that when tensile, compressive or single cycle OL's were inserted periodically within a small cycle below the fatigue endurance limit, the small-stress cycles following those events contribute significantly damage accumulation. Pompetzki and Topper assumed that when an OL/UL was applied periodically in an otherwise small-stress cycle background, that event worked to reduce the crack closure stress and hence enhanced the effective crack driving force. This would persist until the OL/UL effect was diminished and steady state condition was resumed, whereas if the small-stress cycle would instead, be intermittently added into an otherwise larger regular loading pattern. In this case, the larger cycle would not represent OLs but rather they could be regarded as service loads whereas the small-stress cycles would constitute minor loads caused by machine vibration, or other low amplitude external loads.

Ngiau [44] showed the damage contribution from small amplitude cycles in both LCF and HCF regimes for 2024-T351aluminum. Block loads that contain intermittent smallstress amplitude cycle are applied to the regular LCF or HCF loading scheme using two patterns: block to constant (B-C) or constant to block (C-B), i.e. where the blocks precede or follow the regular constant amplitude cycles, respectively. These two patterns are chosen in order to study the contribution of small-stress amplitude cycles in both crack initiation and propagation life. In general, the small stress cycles would affect the initiation life more than the propagation life in the $\mathrm{B}-\mathrm{C}$ sequence, while the opposite 
effect might prevail in the $\mathrm{C}-\mathrm{B}$ sequence. He showed that having intermittent small cycles with amplitude as low as $50 \%$ the endurance limit with regular low cycle fatigue (LCF) or high cycle fatigue (HCF) service loading conditions can indeed be very damaging for both initiation and propagation life. In his study, he has compared damage between $100 \%$ endurance to $50 \%$ endurance limit for HCF and LCF. He found that even $50 \%$ of fatigue limit cycles are also creating significant damage. From above study, it is noted that small cycle up to $50 \%$ of fatigue limit are damaging.

\subsubsection{Description:}

The above discussed effect can be explained from Figure 3.7. This figure shows the relation between stress value with number of cycles to failure. Curve $A(A-B)$ consist of stress values above the endurance limit (it means that value below fatigue limit are neglected) and Curve B (A-C) consists of values above and below the fatigue limit. It is observed that the Curve A has longer life than Curve B; hence it implies that Curve B has something in addition to Curve $\mathrm{A}$ that has significant effect on life of component. Another part of the study is how much percentage of stress below the fatigue limit is damaging and whether the intensity of that stress below and above fatigue limit is same or not. From the above references, it was found that $50 \%$ of fatigue limit is significantly damaging the life of component, and the intensity is definitely dependent on stress and strain amplitudes. If the amplitude is more then damage will be more, hence the cycles below the fatigue limit are damaging but not in the same amount as damage caused by cycles above the fatigue limit.

\subsubsection{Implementation:}

In order to implement the above effect, it is important to identify a limit below which the stresses do not have significant damaging effect. It is found that cycles below $50 \%$ fatigue limit do not effect significantly. Keeping this point in mind, an equation is developed to implement the above effect:

$$
\text { if }\left\{\begin{array}{c}
\sigma_{a i}<0.5 \sigma_{c} \text { then } \sigma_{a}=0 \\
\sigma_{a i} \geq 0.5 \sigma_{c} \text { then } \sigma_{a}=\sigma_{a i}
\end{array}\right.
$$


Here $\sigma_{a i}$ is instantaneous stress amplitude, $\sigma_{a}$ is stress amplitude and $\sigma_{c}$ is endurance limit or fatigue limit. This equation explains that if the stress value is above $50 \%$ of endurance limit then instantaneous value will be considered as stress amplitude otherwise if the stress value is below $50 \%$ of fatigue limit the value becomes zero. In the same regards, Figures (3.81-3.84) prove that the model is working as per equation (3.24). In Figure 3.8 stresses above the fatigue limit are taken and corresponding number of cycles are obtained for constant as well as variable amplitude loading. Similarly stresses at fatigue limit, stresses at $50 \%$ of fatigue limit, and stresses at $20 \%$ of fatigue are shown in Figures 3.8 to 3.11 . Hence the effect of cycles below $50 \%$ fatigue limit on damage accumulation is negligible.

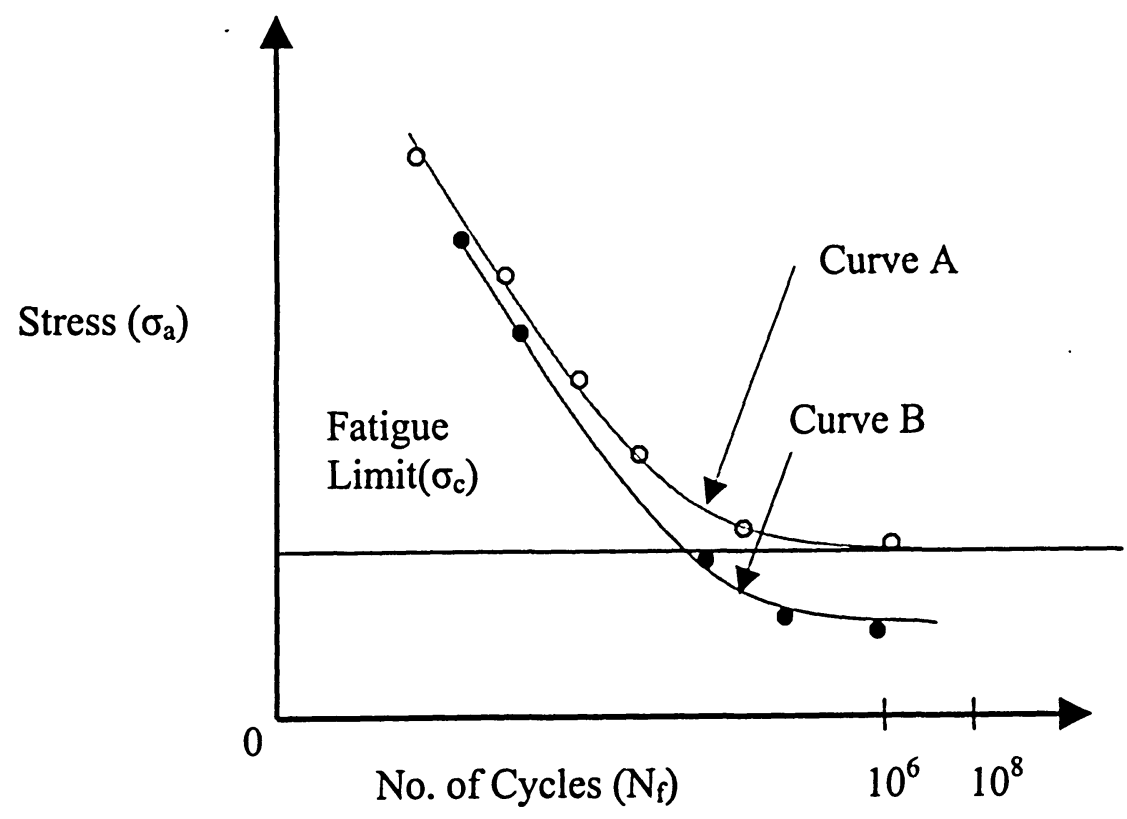

Figure 3.7 Effect of small cycles below fatigue limit [44]. 
All the above-mentioned phenomenological factors are incorporated in the new proposed model using a computer algorithm developed in this study. The details about computer algorithm are explained in the next chapter.

a) Constant amplitude loading:

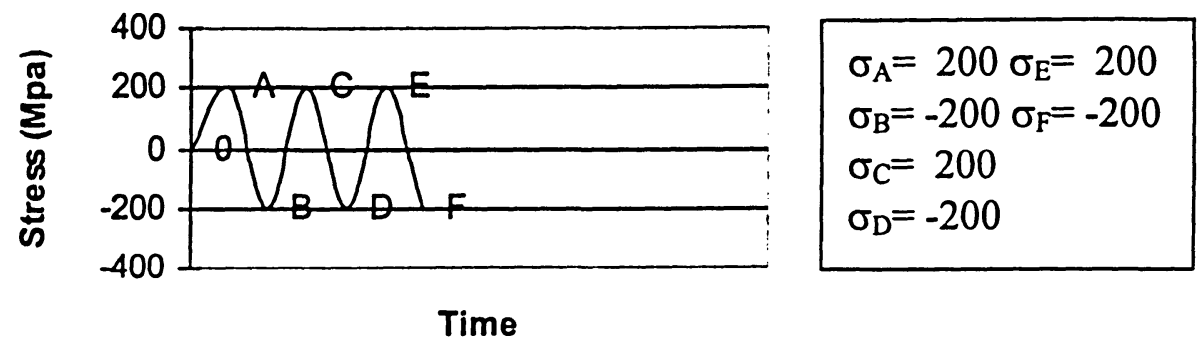

The Number of Cycles required for above stress history: 148000 .

b) Variable amplitude loading:

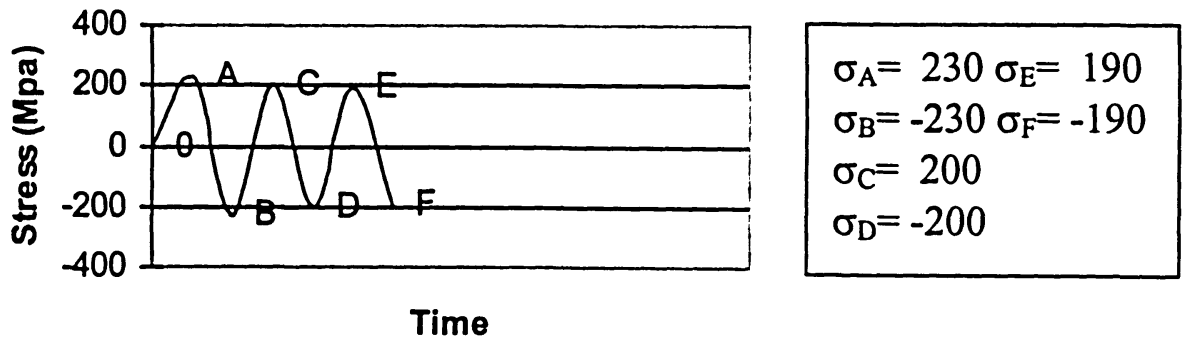

The Number of Cycles required for above stress history: 148000 .

Figure 3.8 Stress value above endurance limit (175MPa). 
a) Constant amplitude loading:

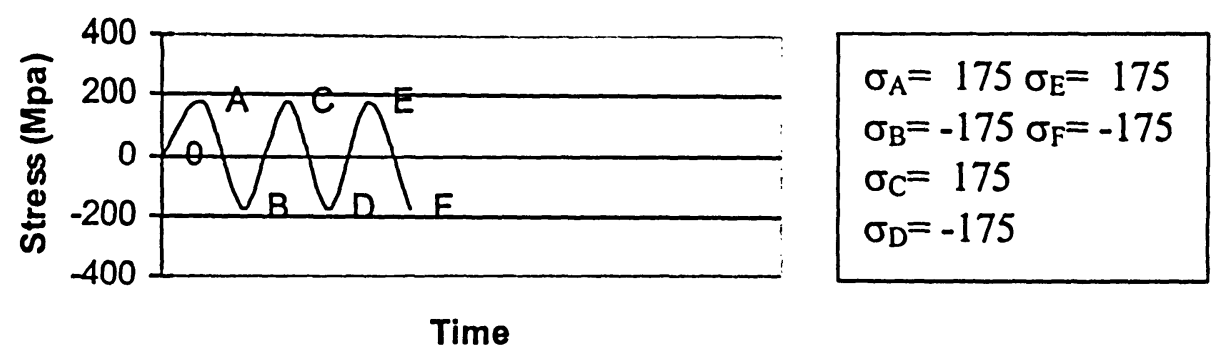

The Number of Cycles required for above stress history: 238000 .

b) Variable amplitude loading:

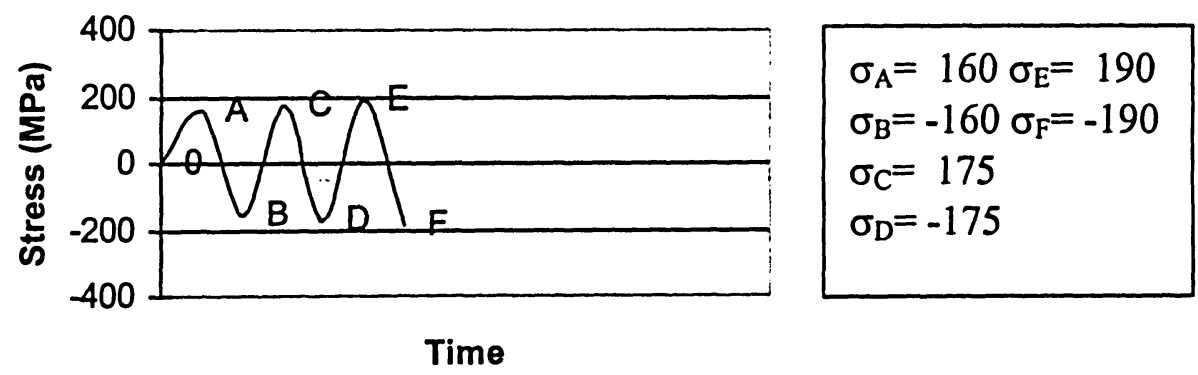

The Number of Cycles required for above stress history: 266500 .

Figure 3.9 Stress value at endurance limit (at 175MPa). 
a) Constant amplitude loading:

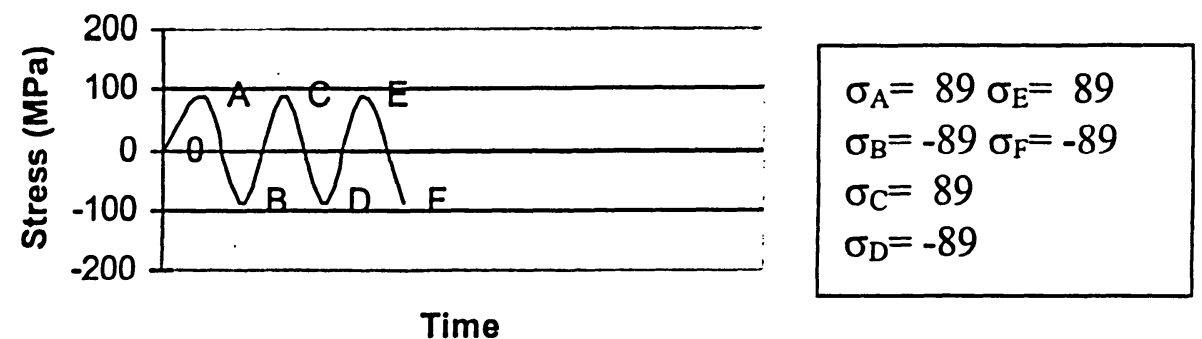

The Number of Cycles required for above stress history: 549000 .

b) Variable amplitude loading:

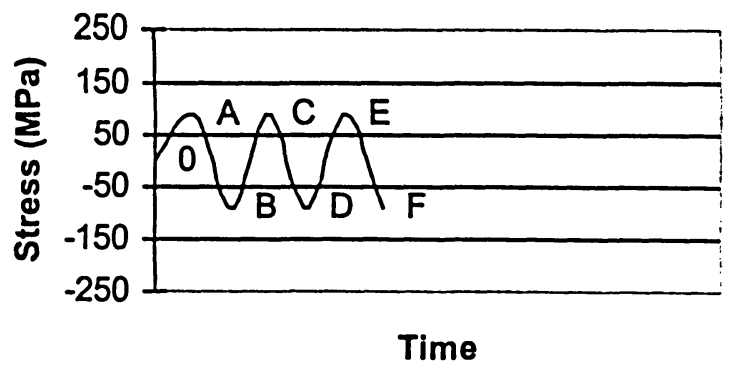

The Number of Cycles required for above stress history: 517500 .

Figure 3.10 Stress value $50 \%$ below endurance limit. 
a) Constant amplitude loading:

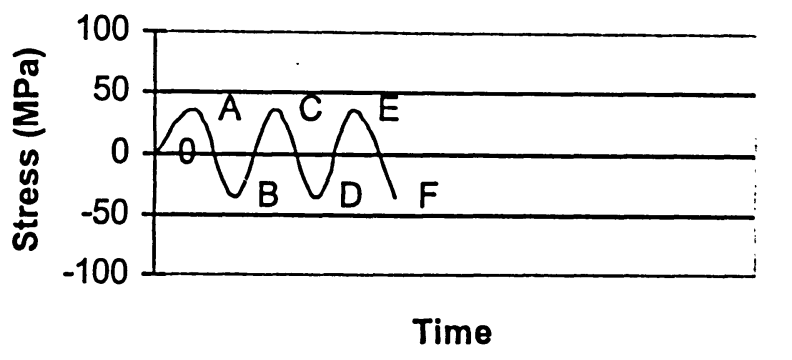

$$
\begin{aligned}
& \sigma_{A}=35 \sigma_{E}=35 \\
& \sigma_{B}=-35 \sigma_{F}=-35 \\
& \sigma_{C}=35 \\
& \sigma_{D}=-35
\end{aligned}
$$

The Number of Cycles required for above stress history: 549000 .

b) Variable amplitude loading:

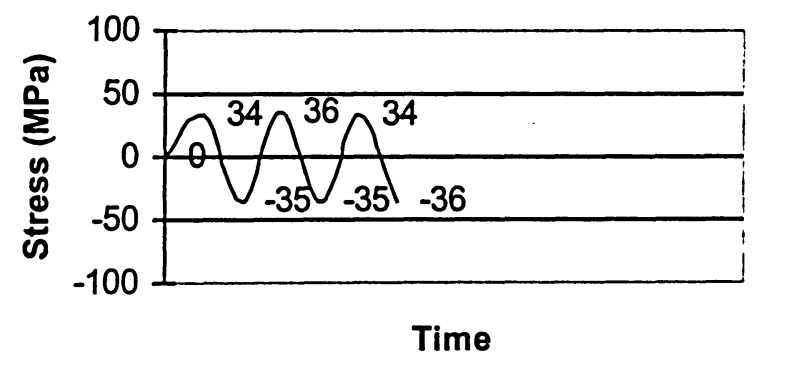

$$
\begin{aligned}
& \sigma_{A}=34 \sigma_{E}=34 \\
& \sigma_{B}=-35 \sigma_{F}=-36 \\
& \sigma_{C}=36 \\
& \sigma_{D}=-35
\end{aligned}
$$

The Number of Cycles required for above stress history: 549000 .

Figure 3.11 Stress value $20 \%$ below endurance limit. 


\section{CHAPTER FOUR}

\section{Algorithm of Fatigue Damage Analysis}

\subsection{Description of Algorithm:}

A computer algorithm and program is developed as shown in Figure 4.1 and 4.2 to find the life of any metallic components subject to fatigue cyclic loading. The program runs at four different stages. Each stage represents loading and unloading alternatively. First two stages run only once at initial time and third and fourth stages run in a loop till the last input is used. The program starts with initialization of axial and shear strength coefficient, axial and shear ductility coefficients, modulus of elasticity, cyclic plastic coefficient, cyclic elastic and plastic exponents, axial and shear stress ranges, axial and shear strain ranges, shear modulus, fatigue limit or endurance limit, maximum stress and minimum stress. The program then asks for first input data from hardware. Stress values derived from experimental is divided into equal intervals as to draw a hysteresis loop. All the stress values between zero and first data are applied in to Ramberg-Osgood equation 3.21 to obtain corresponding total strain values, which will complete the 0 -A section of Figure 3.5. The cyclic stress is used to calculate elastic strain by dividing cyclic stress amplitude by modulus of elasticity. The difference between total strain and elastic strain will give plastic strain value. Effective Poisson's ratio is calculated by utilizing elastic and plastic strain and known elastic and plastic Poisson's ratio. Once these values are obtained three principal strains are calculated from the equations 3.5 to 3.7. These three principal strain values will form three strain Mohr's circles. According to the definition of the critical plane, largest Mohr's circles during loading and unloading reversals will be considered for calculation. Next step is to calculate the principal stresses from input data. 
These three principal stresses will give three stress Mohr's circles and the largest one is considered for calculation. The program now asks hard drive for next available value. The difference between this value and last value obtained from hardware is divided into equal intervals to plot hysteresis for A-B section of Figure 3.5. These stress values will be plugged into unloading Ramberg-Osgood equation (3.22) to calculate strain values. The maximum value obtained by general Ramberg-Osgood equation, from stage 1 , is used in un-loading Ramberg-Osgood equation in stage 2 . From this equation, total strain value is obtained. The above mentioned steps are then utilized to calculate elastic strain, plastic strain, effective Poisson's ratio and finally loading and unloading largest Mohr's circles. The stage one represents loading and unloading. So at this stage largest strain Mohr's circle for loading and unloading are used to calculate normal strain range and shear strain range. The normal strain range $\left(\Delta \sigma_{n}\right)$ is shown in Figure 3.2. It is the horizontal distance between maximum point on loading to minimum point on unloading. The maximum shear strain range $\left(\Delta \varepsilon_{n}\right)$ is shown in Figure 3.2. It is the vertical distance between maximum point on loading Mohr's circle to minimum point on unloading Mohr's circle.

Next step is to calculate mean stress by taking an average of two data. The calculated value is substituted into equation (3.19) to calculate the damage function from all the four ranges so far calculated and four known coefficient. This damage function is added to imaginary function in order to get the addition of all the damage function. Now program asks for third input data. This input data is checked for $50 \%$ endurance limit. If the data is less then $50 \%$ endurance limit, then it will ask for next data otherwise it will pass on the data to next step. At this point, stage three and four are calculated in the same manner as one and two. Except in stage one, original Ramberg-Osgood equation is used whereas in stage three, loading Ramberg-Osgood is used. Therefore, damage function is calculated from stage three and stage four value is added to previous damage function with the help of imaginary function initialized in the beginning. Remaining values are calculated as stage three and four from repeated loop are added to the previous damage function until all the input is used. The damage function is then equated to Coffin-Manson equation to estimate the number of cycles. 


\subsection{Conceptual Flow Chart:}

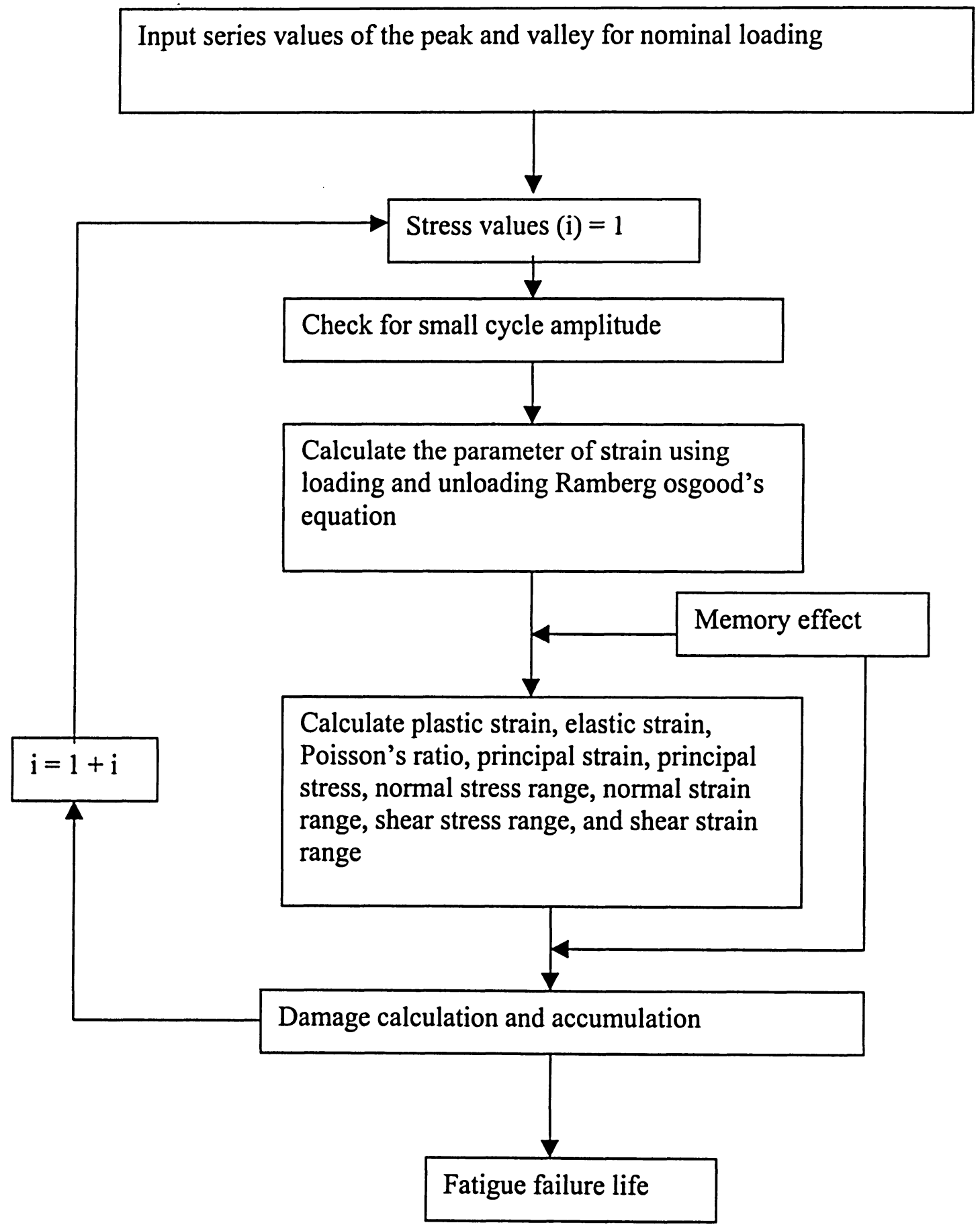

Figure 4.1 Conceptual Flow Chart. 


\subsection{Computer Program Flow chart (Appendix-B):}

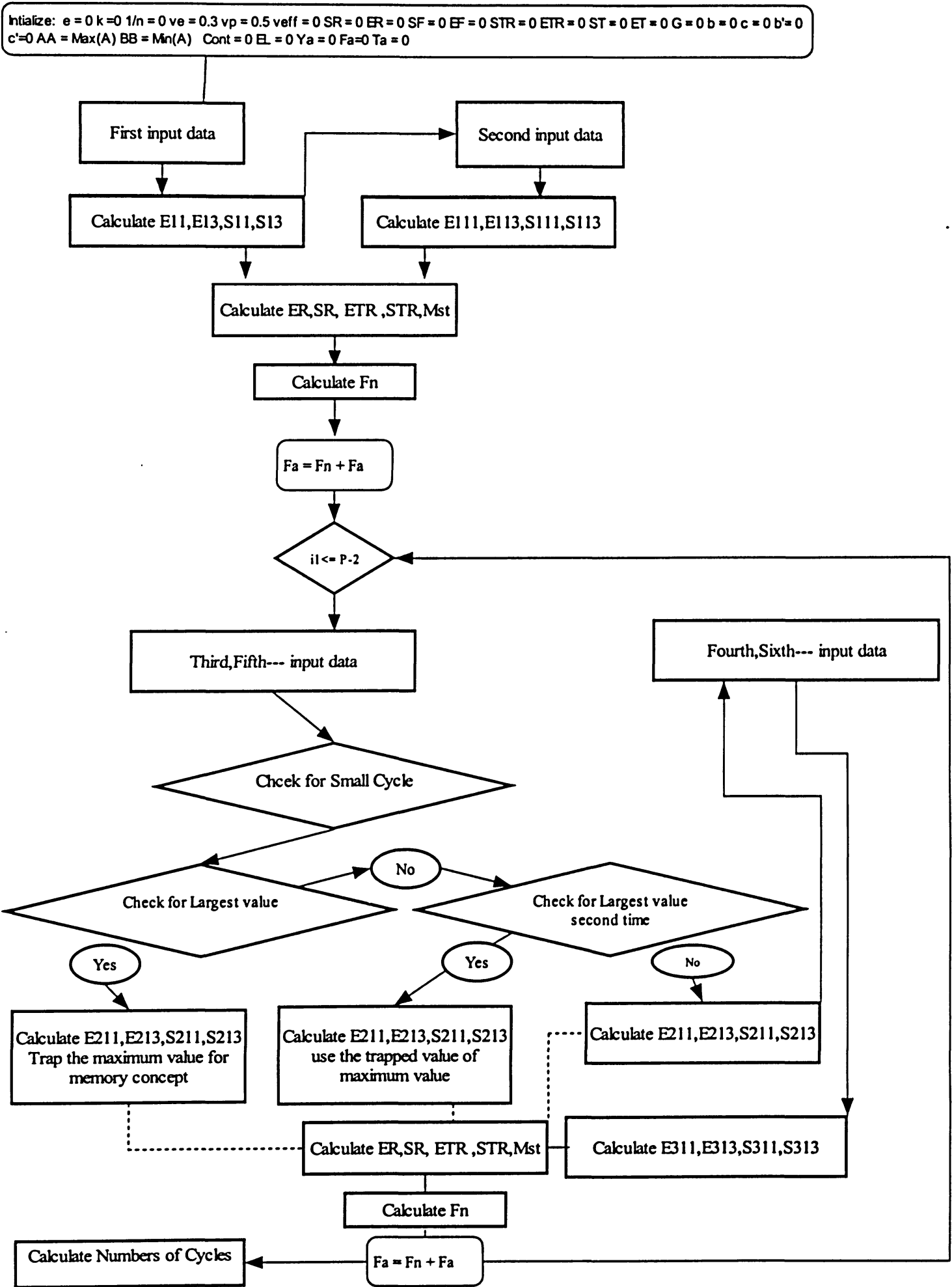

Figure 4.2 Computer Flow Chart. 


\section{CHAPTER FIVE}

\section{Fatigue Damage Model Evaluation and Results}

This chapter deals with evaluation of fatigue damage model by different experimental data available in literature. Data are first converted to terms required in the model and accordingly the values are substituted in the model. This chapter evaluates the model initially with constant amplitude loading, then with step loading and finally with variable amplitude loading conditions.

\subsection{Constant loading History:}

To evaluate fatigue damage model for constant amplitude loading, the experimental data of Ngiau [44] for uniaxial constant and step loading have been used. Ngiau has tested 2024-T351 aluminum alloy, which is one of the alloys that the automotive industry has considered to be a plausible lightweight substitute for steel. Specimens were machined from $19.05 \mathrm{~mm}$ diameter bars. The specimens conform to ASTM E466-82 standards and have a gauge diameter of $12.7 \mathrm{~mm}$, continuous radius of $152.4 \mathrm{~mm}$, a grip cross sectional diameter of $19.05 \mathrm{~mm}$ and a total length of $228.6 \mathrm{~mm}$. Testing was conducted using a MTS 810 uniaxial test frame. All tests were fully reversed and load-controlled. The load history applied to the model is shown in the Figure 5.1.1, the mechanical and cyclic properties used for uniaxial constant amplitude loading are listed in Table A-1. Constant amplitude tests were performed to determine the stress amplitude versus fatigue life (S$\mathrm{N}$ ) diagram for the material investigated. The S-N diagram is obtained as shown in Figures 5.1.2. From this Figures it can be noticed that during shorter life, the model shows conservative life up to $10^{3}$, then in the intermediate life $10^{3}$ to $10^{4}$, the model 
shows non-conservative and then again the life is conservative in longer life. In the same figure Miner's rule is included to compare different approaches.

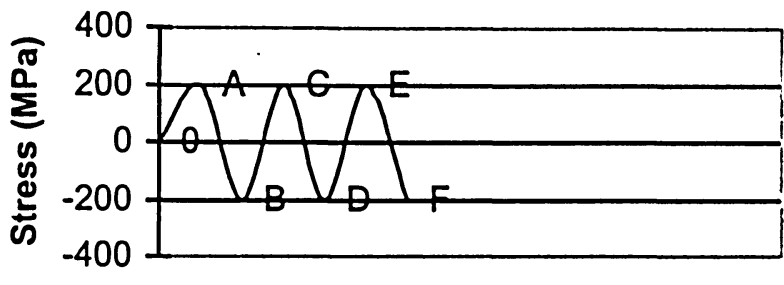

Constant Loading [44]

$\sigma_{\mathrm{A}}=200 \sigma_{\mathrm{D}}=-200$

$\sigma_{\mathrm{B}}=-200 \sigma_{\mathrm{E}}=200$

$\sigma_{C}=200 \sigma_{F}=-200$

Time

Figure 5.1 Constant amplitude loading History by Ngiau [44].

Figure 5.2 shows four different cases, Case ' $a$ ' shows predicted data without any phenomenological factors included. Case ' $b$ ' represents predicted data including the sequence effect. Case ' $c$ ' describes predicted data with sequence and memory effects. Case' $d$ ' presents predicted data including sequence effect, memory effect and small cycle effect.

It can be seen from the figures presented that there is no difference between all four cases. This is so because; in constant loading there is no effect of sequence, memory and small cycle effects. Figure 5.3 shows a comparison between the proposed model and experimental data. The proposed fatigue analysis shows a good agreement with experimental data for uniaxial constant loading. The diagonal dashed line in figure 5.3 represents a comparison line and solid lines indicate a ratio of variation between predicted and experimental lives as compared with the dashed line. The maximum variation is 3.95 in this case. 


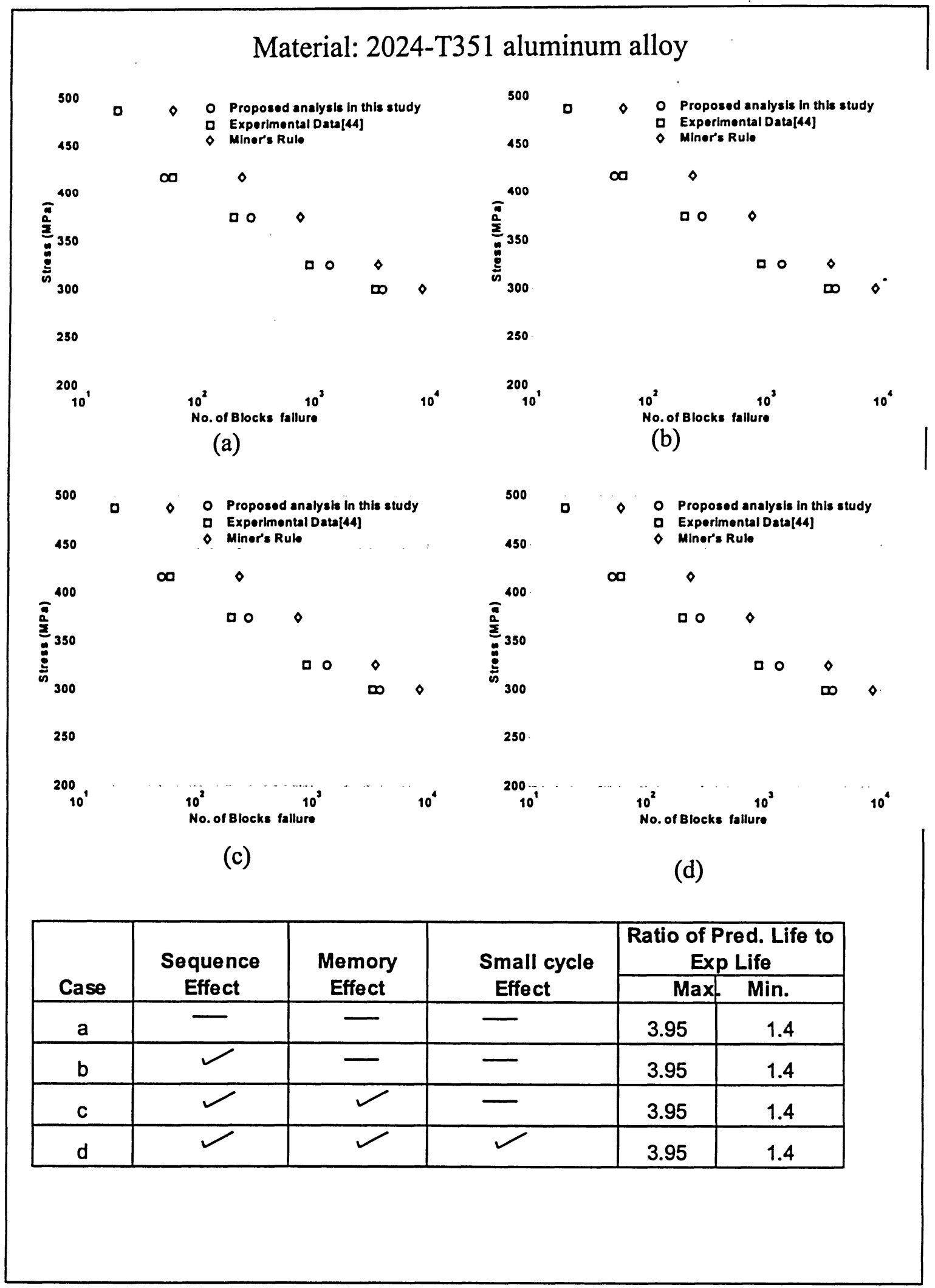

Figure 5.2 Comparison of proposed fatigue damage approach and Miner's rule with experimental fatigue life data of 2024-T351 aluminum alloy. 


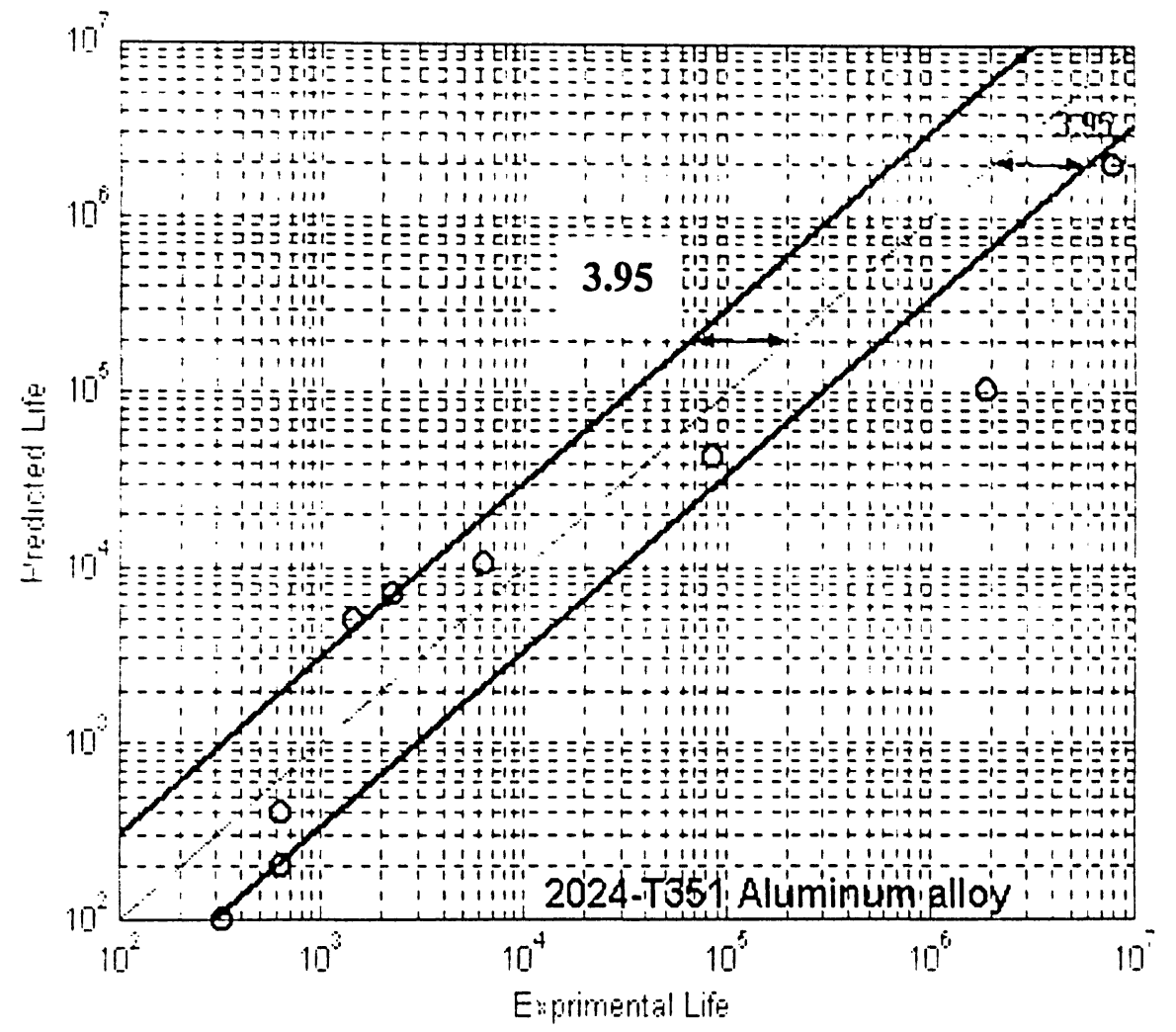

Figure 5.3 Experimental and predicted fatigue lives in Al 2024-T351alloy.

\subsection{Step Loading History:}

The step loading history used to evaluate the proposed approach in this thesis is shown in figures 5.4 and 5.5. The figure 5.4 shows that initially five LCF cycles of $420 \mathrm{MPa}$ is followed by 1000 constant amplitude cycles (with amplitude of $175 \mathrm{MPa}$ ). When the above mentioned history is applied to the proposed approach it gives little conservative life of 4250 cycles. Another case is $100 \mathrm{HCF}$ cycles of $220 \mathrm{MPa}$ is followed by 1000 constant amplitude cycles (with amplitude of $175 \mathrm{MPa}$ ). The experimental results from these type of loading is 100,000 cycles and the predicted life using the proposed approach found to be 134,670 cycles. 


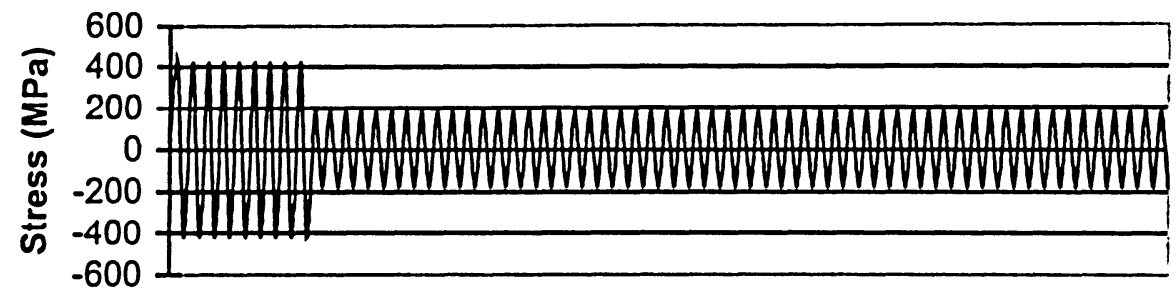

Time

Figure 5.4 LCF cycles loading history by Ngiau [44].

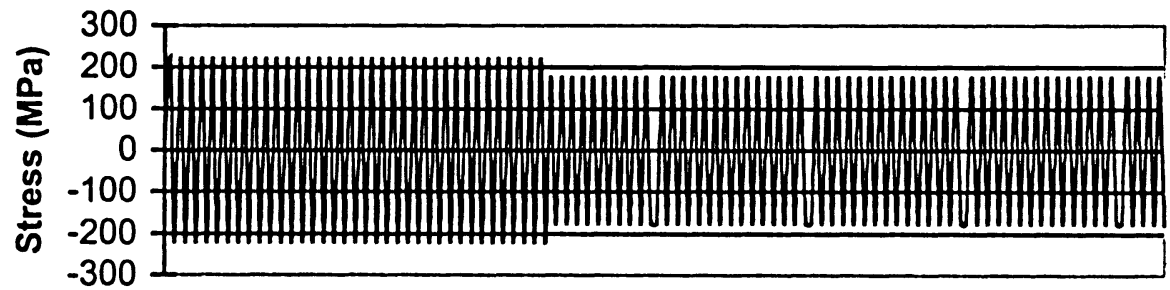

Time

Figure 5.5 HCF cycles loading history by Ngiau [44].

\subsection{Variable Amplitude Loading History :}

The entire service loads are generally variable or random in nature. This section will evaluate the capability of the fatigue damage approach presented in this thesis for variable amplitude loading condition. The variable loading histories and life data by Kilman [40] conducted on cylindrical specimens of low carbon steel $(0.4 \mathrm{wt} \% \mathrm{c})$ used in this study to evaluate the damage model in this thesis. The test specimens were loaded in the stress-controlled mode using an MTS computer controlled fatigue system. The loading block was made up of 113 randomly arranged cycles. In order to use the experimental data provided by Kilman. variable loading history has been transformed as per maximum stress value in S-N curve. Figure 5.6 shows the loading history applied by Kilman [40]. 


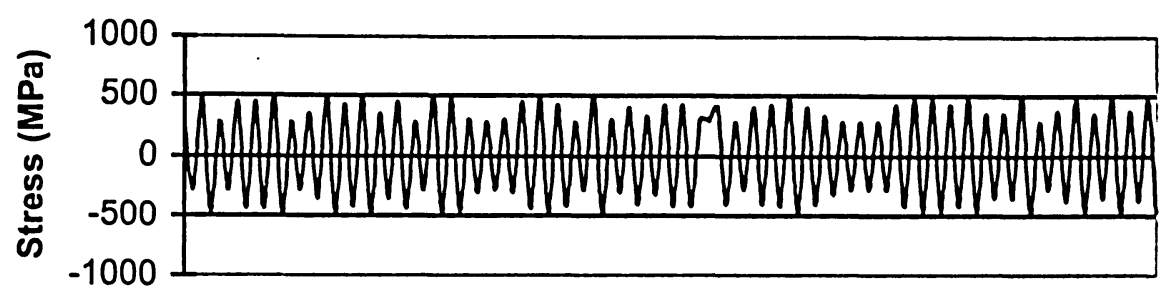

Time

Figure 5.6 Variable loading history by Kilman [40].

Figure 5.7 shows four different cases, Case ' $a$ ' shows predicted data without including any phenomenological factors. Case ' $b$ ' represents predicted data with sequence effect. Case ' $c$ ' describes predicted data with sequence effect and memory effect. Case 'd' presents predicted data with sequence effect, memory effect and small cycle effect. It can be observed from the table given in the Figure 5.3.2 that maximum variation is calculated as the maximum difference between experimental data to data obtained from proposed analysis and minimum variation is calculated as minimum difference between experimental data and data obtained from proposed analysis at any given time between experimental and predicted life data. For Case ' $a$ ' the maximum variation is 3.14 and minimum variation is 1.5 . By introducing sequence effect the maximum variation reduces to 2.96 and the minimum variation reduces to 1.5 . Maximum variation further reduces to 1.48 and 1.36 with introduction of memory and small cycle effects. Hence it can be deduced from the table that introducing phenomenological factors in the study has provided more favorable results. 


\section{Material: Low Carbon Steel $(0.4 \mathrm{wt} \% \mathrm{C})$}
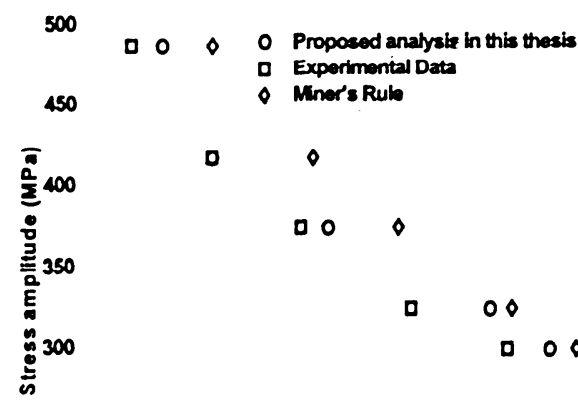

250

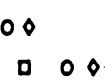

$\begin{array}{lll}200 & & \\ 10^{2} & 10^{3} & 10^{3} \\ & \text { No. of Blocks failure } & 10^{4}\end{array}$

(a)

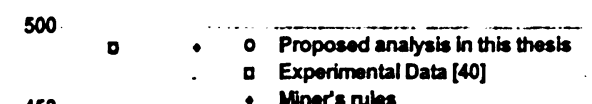

- Miner's rules

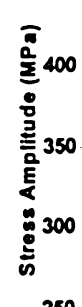

250

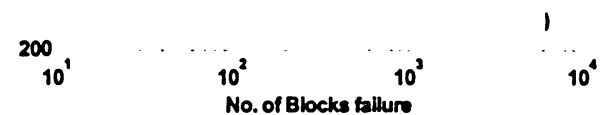

(c)
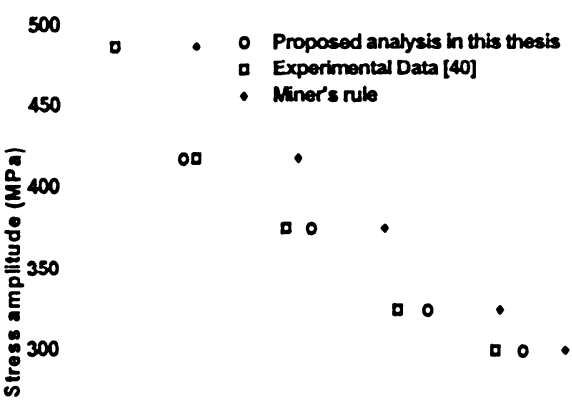

250

200

$10^{2} \quad 10^{3}$

No. of Blocks failure

(b)

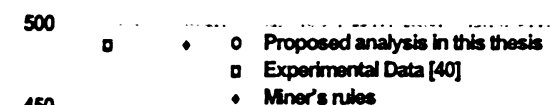

450

0 -

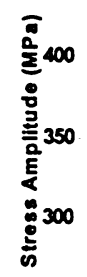

250

200
10

$10^{2}$
Na. of Blocks tallure

(d)

\begin{tabular}{|c|c|c|c|c|c|}
\hline \multirow[b]{2}{*}{ Case } & \multirow{2}{*}{$\begin{array}{c}\text { Sequence } \\
\text { Effect }\end{array}$} & \multirow{2}{*}{$\begin{array}{l}\text { Memory } \\
\text { Effect }\end{array}$} & \multirow{2}{*}{$\begin{array}{c}\text { Small cycle } \\
\text { Effect }\end{array}$} & \multicolumn{2}{|c|}{$\begin{array}{l}\text { Variation of Pred. } \\
\text { Life }\end{array}$} \\
\hline & & & & Max. & Min. \\
\hline $\mathbf{a}$ & - & - & - & 3.14 & 1.5 \\
\hline b & $\gamma$ & - & - & 2.92 & 1.5 \\
\hline c & $\checkmark$ & $\checkmark$ & - & 1.48 & 1 \\
\hline d & 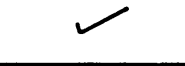 & 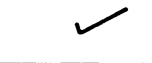 & $\checkmark$ & 1.36 & 1 \\
\hline
\end{tabular}

(e)

Figure 5.7 Comparison of proposed fatigue damage approach and Miner's rule with experimental fatigue life data of Low carbon steel. 
Figure 5.7 shows relationship between the alternating stress and number of cycle. It also shows good relationship between experimental and predicted life data from proposed model. Figure 5.8 shows that experimental life data agrees with predicted life calculated from proposed damage analysis and maximum variation in this case is 1.36 .

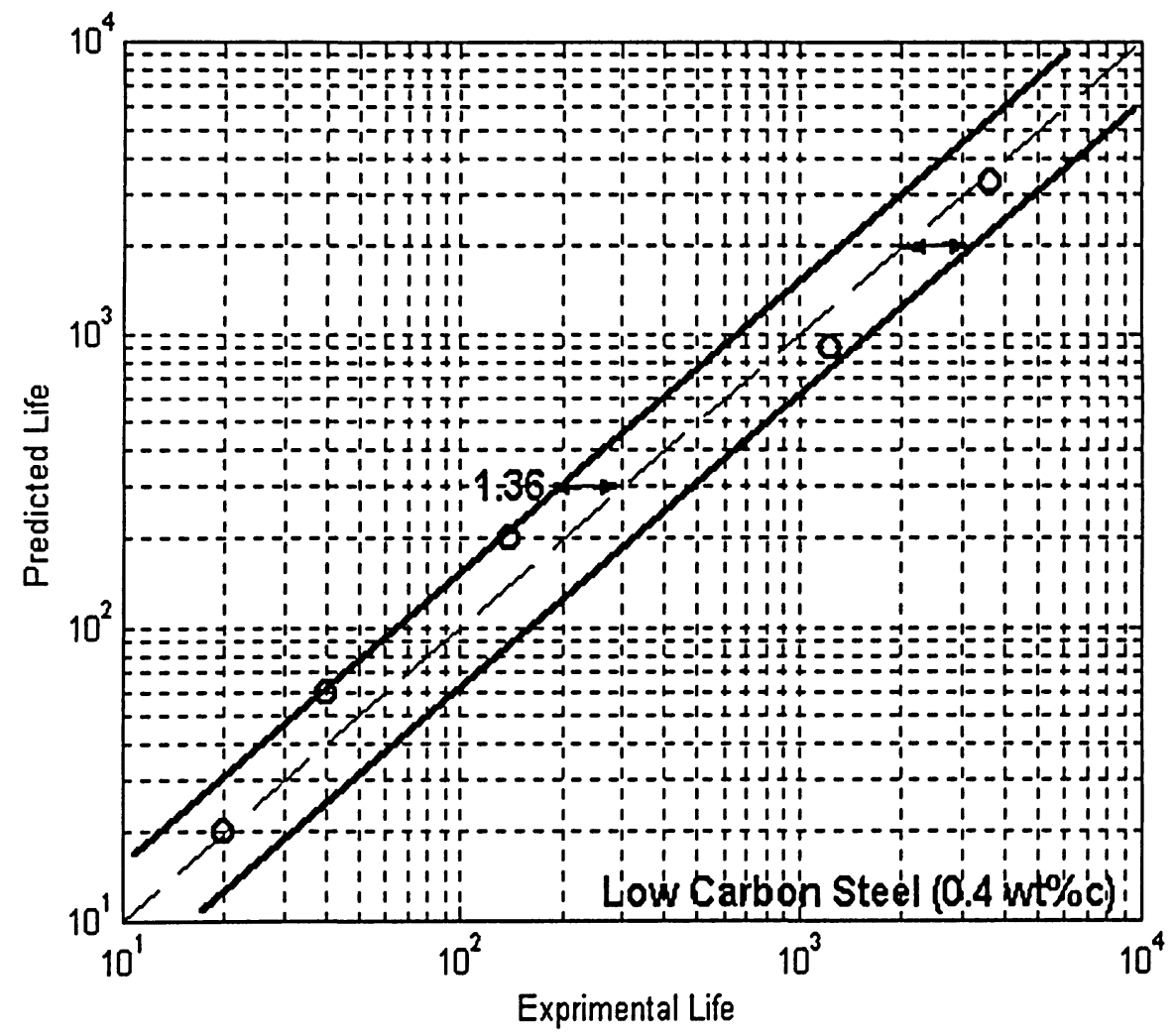

Figure 5.8 Experimental and calculated fatigue lives for low carbon steel

Wu et al. [49] has carried out experiments on 7075-T761 aluminum alloy and specimens were made in accordance to ASTM E606 standard. The material has a yield stress of 503 $\mathrm{MPa}$, ultimate strength of $647 \mathrm{MPa}$ and Young's modulus $74 \mathrm{GPa}$. An MTS axial- 
torsional servo-hydraulic test machine was used to perform fatigue tests. Figure 5.9 presents variable amplitude loading spectrum used by $\mathrm{Wu}$ et al. [48].

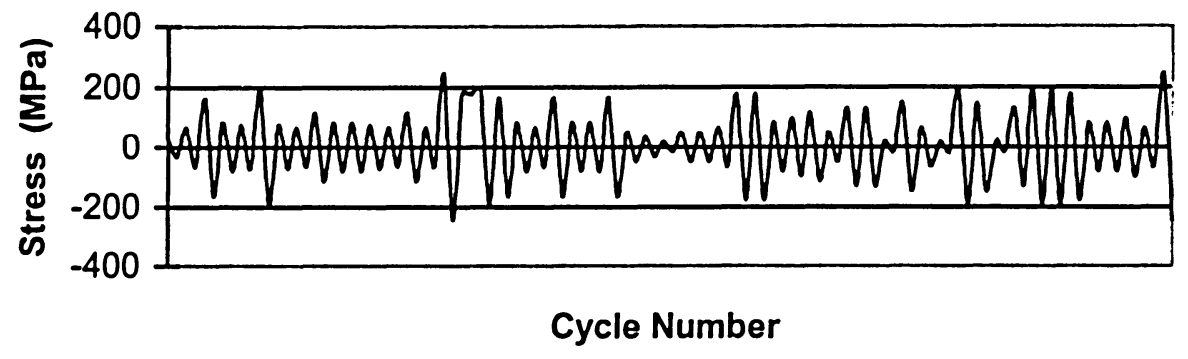

Figure 5.9 Variable amplitude loading history byWu et al. [48].

Figure 5.10 shows four different cases. In this figure, Case a shows predicted data without considering the effect of any phenomenological factors. Case b represents predicted data including loading sequence effect. Case $\mathrm{c}$ presents predicted data including sequence and memory effects. Case $d$ presents predicted data with sequence effect, memory effect and small cycle effect. The table compares experimental and predicted life data. The maximum variation is calculated as maximum difference between experimental and predicted life data. The maximum variation is 3.56 . This gradually reduces to 2.46 with introducing the sequence effect. It further reduces to 1.48 with including the memory effect. Finally when small cycle effect is introduced it further gives better results by reducing the value to 1.36 . 


\section{Material: 7075-T761 aluminum alloy}

350

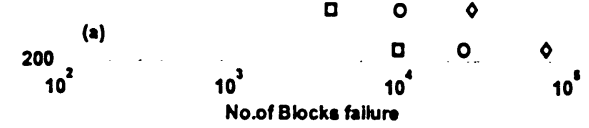

(a)

350

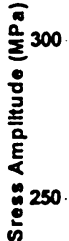

Proposed analysis in this thesis

D Experimental Data [48]

- Miner's Rule

- $\infty$

00

$\begin{array}{ccc}0 & 0 & 0 \\ 10^{4} & & 10\end{array}$

- 0 Proposed analysis in this thesis

D Experimental Data [48]

$\checkmark$ Miners Rule

- $\infty$

$\begin{array}{lll}0 & 0 & 0 \\ 0 & 0 & 0\end{array}$

$\begin{array}{ccccc}200 & \text { (c) } & 0 & 0 & 0 \\ 10^{2} & 10^{3} & 10^{4} & & 10^{5} \\ & & \text { No.of Blocks fallure } & & \end{array}$

(c)

\begin{tabular}{|c|c|c|c|c|c|}
\hline & \multirow{2}{*}{$\begin{array}{c}\text { Sequence } \\
\text { Case }\end{array}$} & \multirow{2}{*}{$\begin{array}{c}\text { Memory } \\
\text { Effect }\end{array}$} & \multirow{2}{*}{$\begin{array}{c}\text { Small cycle } \\
\text { Effect }\end{array}$} & \multicolumn{2}{|c|}{\begin{tabular}{c} 
Variation of Pred. \\
\cline { 5 - 6 }
\end{tabular}} \\
\cline { 5 - 7 } & Life & Max. & Min. \\
\hline a & - & - & - & 3.56 & 2.14 \\
\hline b & $\nearrow$ & - & - & 2.46 & 1.5 \\
\hline c & $\nearrow$ & $\checkmark$ & - & 1.48 & 1 \\
\hline d & $\nearrow$ & $\checkmark$ & $\checkmark$ & 1.36 & 1 \\
\hline
\end{tabular}

Figure 5.10 Comparison of proposed fatigue damage approach and Miner's rule with experimental fatigue life data of 7075-T761 aluminum alloy.

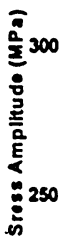

O Proposed anatyets in this theels

D Experimental Data [48]

a $\infty$

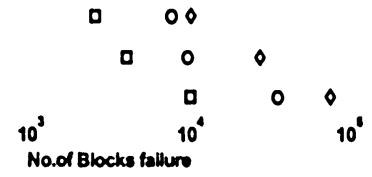

(b)

$350 \quad 0 \quad 0$ Propoeed analyais in this thesis

D Experimental Data [48]

- Mnoro Rulo [48]

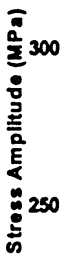

$200 \stackrel{(0)}{10^{2}}$

(d) $10^{3}$ No.of Blocks fallure

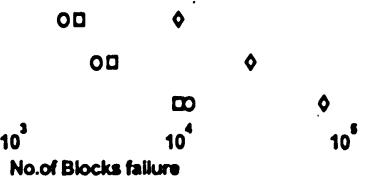
$10^{\circ}$ 
Figure 5.11 shows good relationship between experimental and predicted fatigue life values.

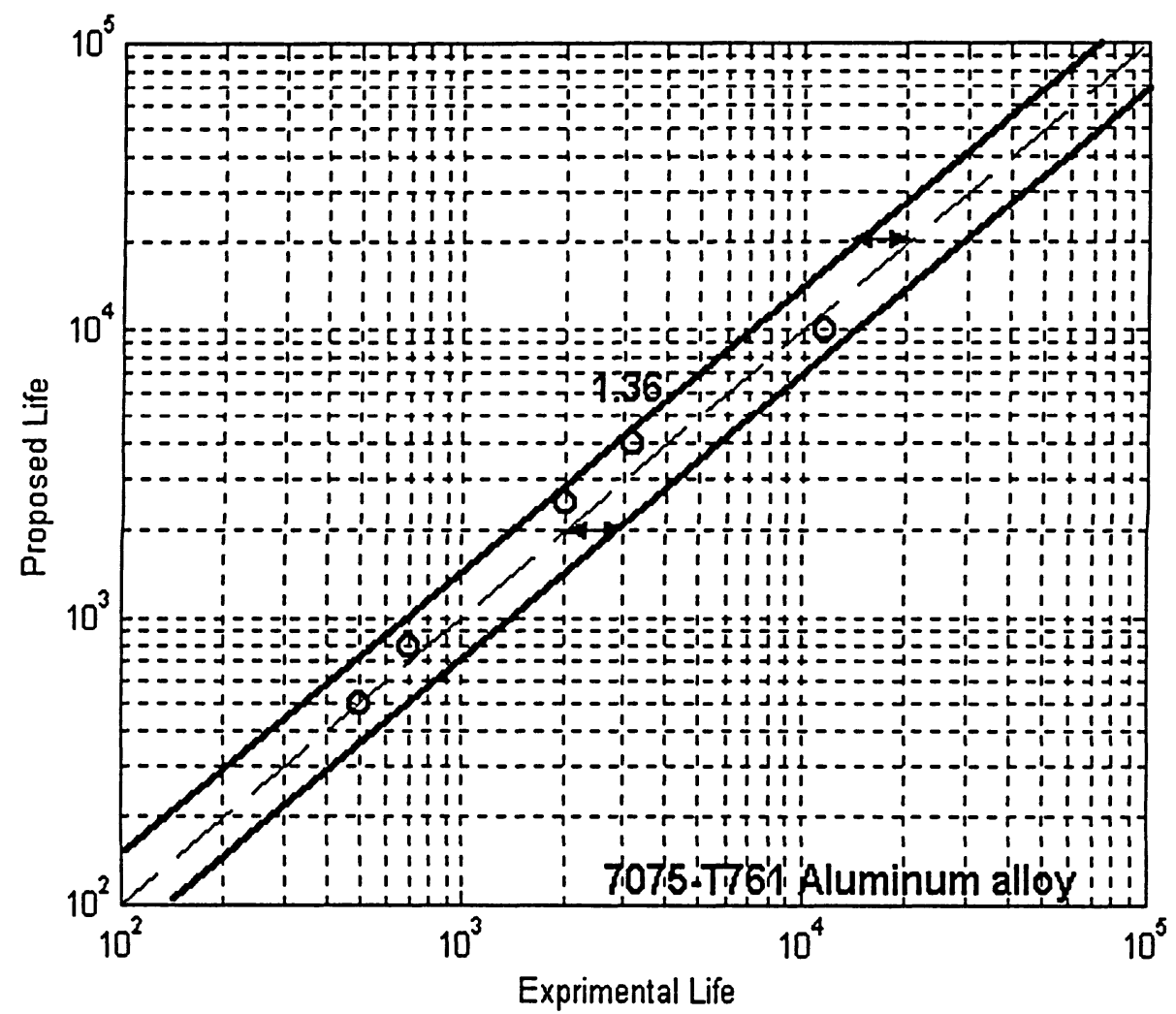

Figure 5.11 Experimental and calculated lives data for Al 7075-T 761 alloy.

Everett [41] has carried out studies on typical commercial transport design spectra for simple block sequence of loads. The material used for this study was 2024-T3 aluminum sheet taken from a special stock of material at the NASA Langley research center, which has been used for fatigue and fracture studies over several decades. The alloy 2024 has been used in the lower wing skin of many commercial transport aircraft. The material has yields strength of $52 \mathrm{ksi}$ and an ultimate strength of $72 \mathrm{ksi}$. The fatigue endurance limit of this material is $18 \mathrm{ksi}$ and the nominal thickness is 0.090 in. the study consists of five different flight types for random loading. Flight number one is the most severe and occurs only once in 5000 flights. Flight number two occurs 13 times, flight number three 
occurs 215 times, and flight number four occurs 1067 times, respectively, in the 5000 cycles sequence. Figure 5.12 presents variable loading history reported by Everett [41].

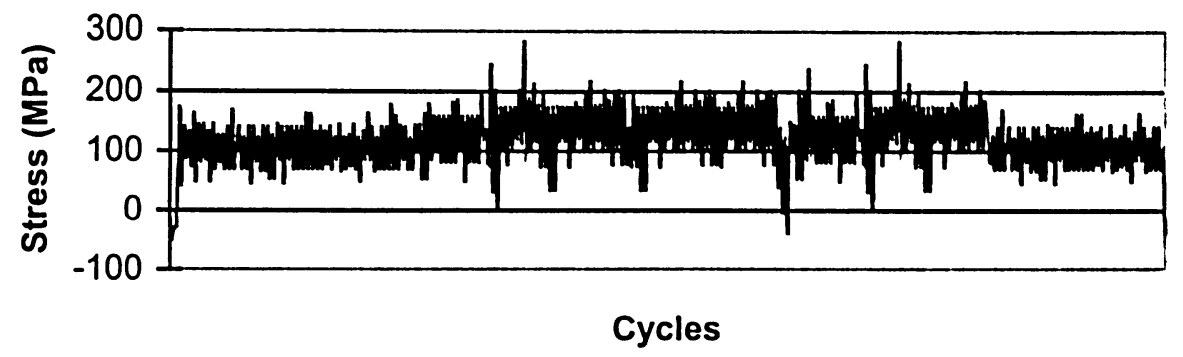

Figure 5.12 Variable amplitude loading history by Everett [41].

In figure 5.13, Case ' $a$ ' presents predicted data with no phenomenological factors. Case ' $b$ ' represents predicted data with sequence effect. Case ' $c$ ' describes predicted data with sequence and memory effects. Case ' $d$ ' presents predicted data with sequence, memory effect and small cycle effect. For Case 'a' the maximum variation is 3.95 and minimum variation is 1.5. By introducing sequence effect the maximum variation reduces to 3.45 and minimum to 1.34. Maximum variation further reduces to 1.75 and 1.67 with introduction of memory and small cycle effect. Hence it can be deduced from the table that introducing phenomenological factors in the study has provided more favorable results. 


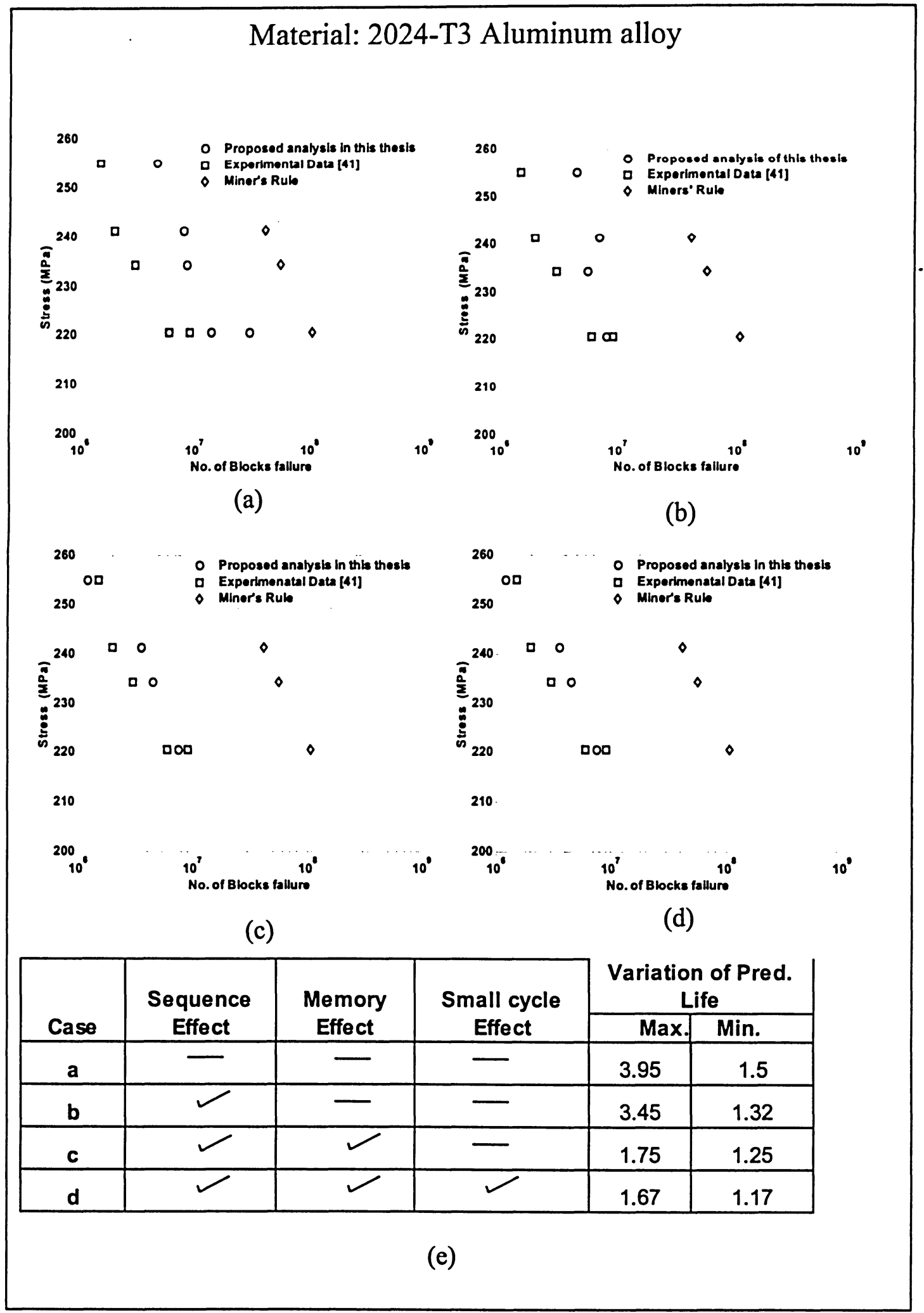

Figure 5.13 Comparison of proposed fatigue damage approach and Miner's rule with experimental fatigue life data of 2024-T3 aluminum alloy. 
Everett has done this study to account for sequence loading effect in commercial transport. The five loading histories were used to find out the life of component. Figure 5.14 shows the first and most severe loading for fatigue damage analysis. It can be seen from the Figure that experimental fatigue life data and calculated life results fall within the maximum variation of 1.67 .

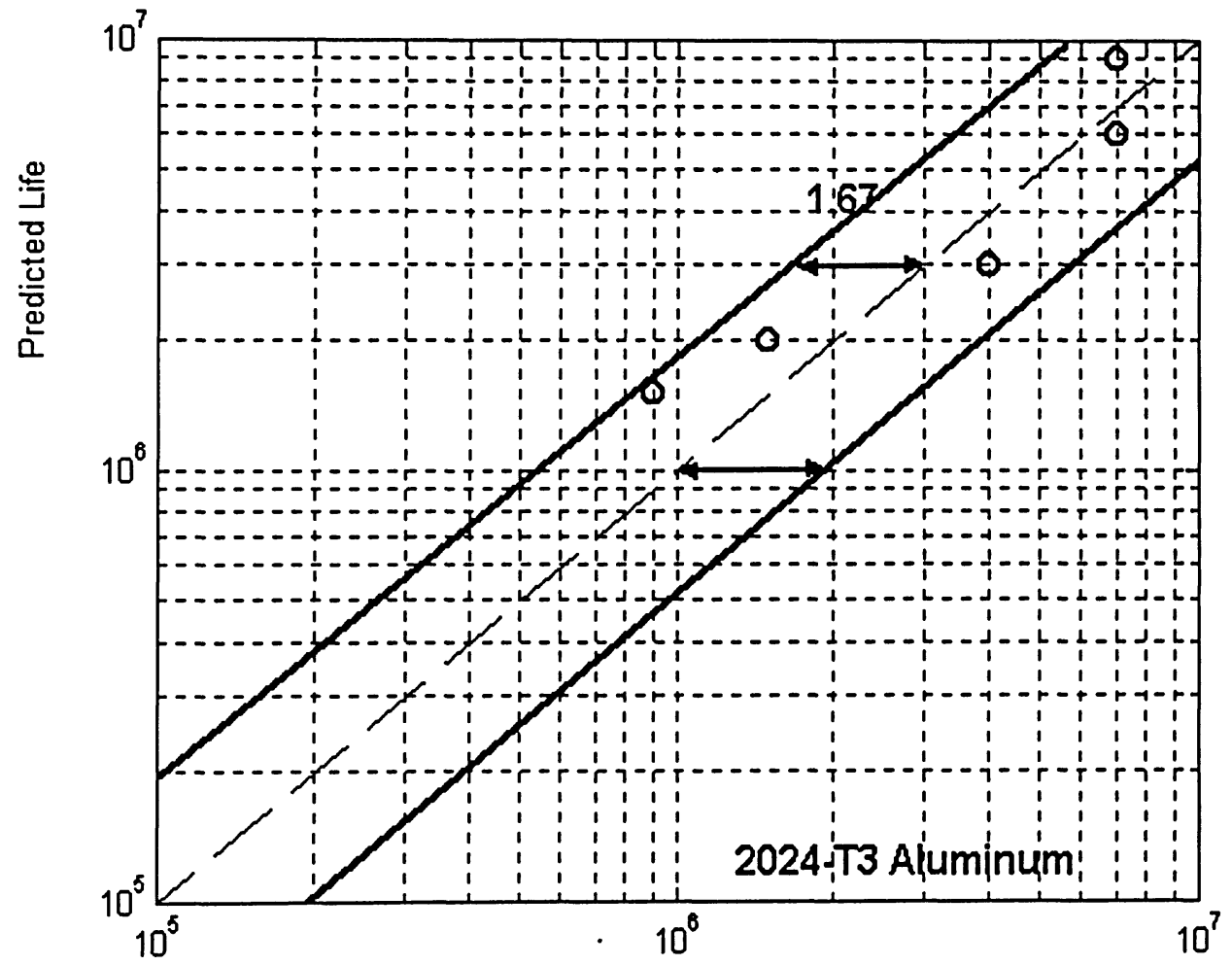

Experimental life

Figure 5.14 Experimental and calculated lives data for Al 2024 T3 alloy.

For steel bridge deck, Agerskov [47] has done investigation by strain gauge measurements on the orthotropic steel deck structure of the Faro Bridge in Denmark. Strain gauge measurements were carried out at 10 different locations in the orthotropic deck. The measurement area is placed at a distance of approximately $8 \mathrm{~m}$ from the simple support of the bridge girder on the nearest bridge pier. The length of the bridge spans is approximately $80 \mathrm{~m}$. This location of the strain gauges means that only local bending 
effect in the deck structure will be registered, whereas the stresses due to global bending in the bridge girder will be negligible. Ten strain gauges are placed in two sections between two transverse diaphragms. One of these sections is in the middle of the longitudinal stiffener span, which has a length of $4 \mathrm{~m}$, and the other section is placed at a distance of $0.5 \mathrm{~m}$ from one of the transverse diaphragms. Four of the strain gauges in each section are placed on the bottom of trapezoidal longitudinal stiffener of the deck plate. The fifth gauge in each section is placed on the butt weld between the trapezoidal stiffener and the deck plate. In this way variable realistic loading data is compared with constant amplitude data used in early studies. Figure 5.15 presents variable loading history used by Agerskov [47].

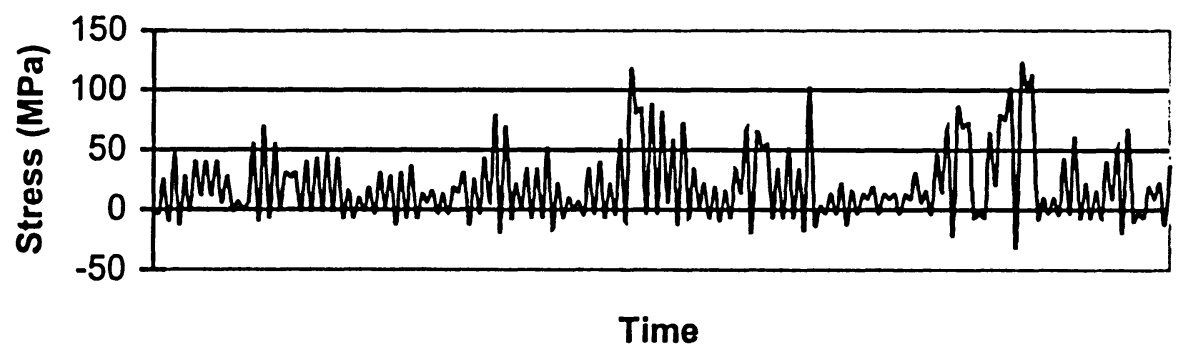

Figure 5.15 Variable amplitude loading history [47].

In figure 5.16, Case ' $a$ ' shows predicted data without any phenomenological factors. Case ' $b$ ' represents predicted data with sequence effect. Case ' $c$ ' describes predicted data with sequence and memory effects. Case ' $d$ ' presents predicted data with sequence effect, memory effect and small cycle effect. The maximum variation is 2.77 ; this gradually reduces to 2.00 with the introduction of sequence effect. It further reduces to 1.92 with introduction of memory effect and finally when small cycle effect is introduced it further gives better results by reducing the value to 1.33 . 


\section{Material: Low Carbon Steel}

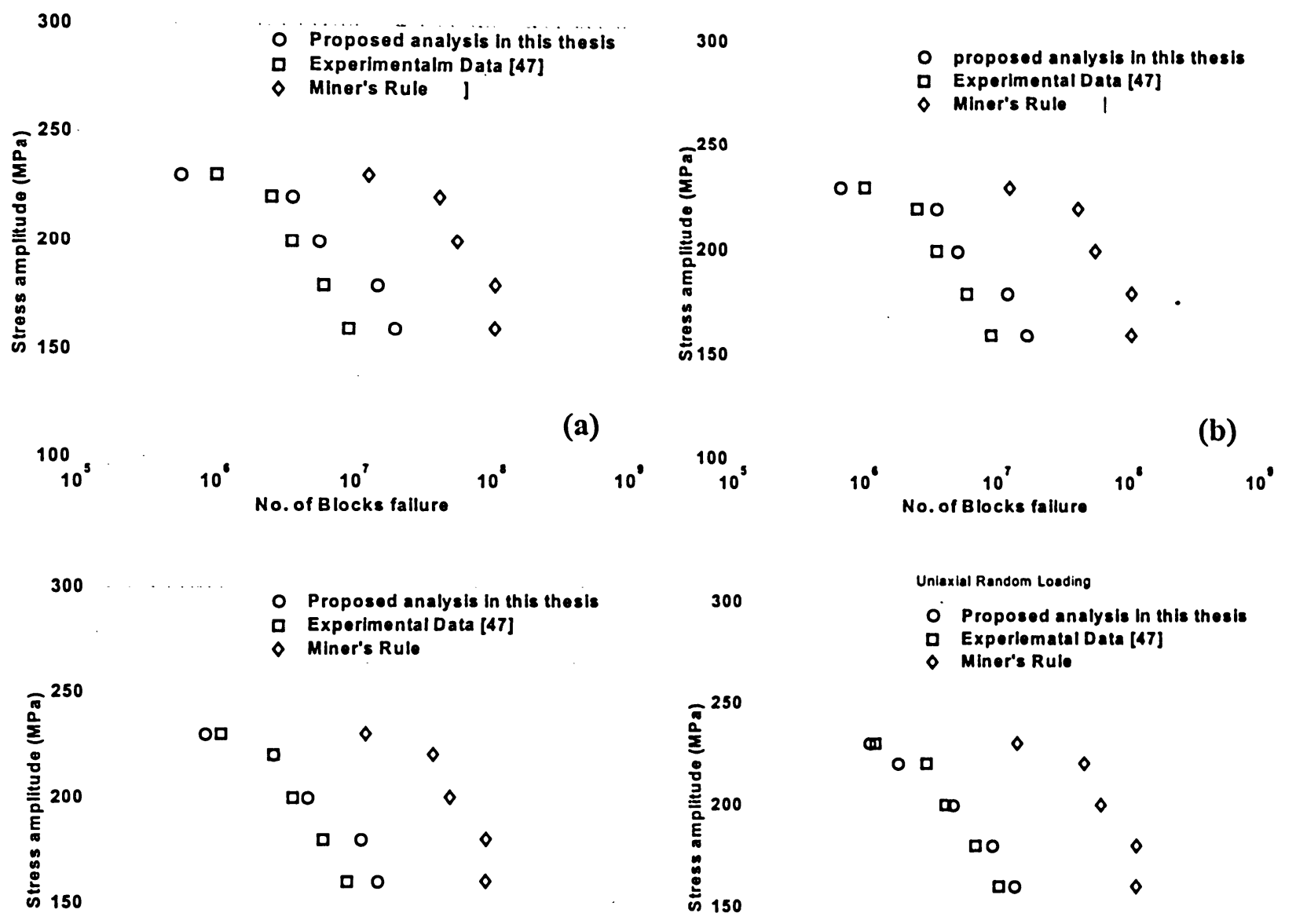

(c)
${ }_{10}^{100}$
$1^{\circ}$ No. of Blocks fallure $10^{7}$
$10^{\circ} \quad{ }^{100} \mathrm{~s}$
$10^{\circ}{ }^{10^{7}}{ }^{\circ}$ No. of Blocks fallure
$10^{\circ}$

(d)

\begin{tabular}{|c|c|c|c|c|c|}
\hline \multirow[b]{2}{*}{ Case } & \multirow{2}{*}{$\begin{array}{c}\text { Sequence } \\
\text { Effect }\end{array}$} & \multirow{2}{*}{$\begin{array}{l}\text { Memory } \\
\text { Effect }\end{array}$} & \multirow{2}{*}{$\begin{array}{c}\text { Small cycle } \\
\text { Effect }\end{array}$} & \multicolumn{2}{|c|}{$\begin{array}{l}\text { Variation of Pred. } \\
\text { Life }\end{array}$} \\
\hline & & & & Max & Min. \\
\hline a & $\longrightarrow$ & $\longrightarrow$ & - & 2.77 & 1.14 \\
\hline b & $\checkmark$ & $\longrightarrow$ & $\longrightarrow$ & 2.00 & 1.4 \\
\hline c & $\checkmark$ & $\sim$ & - & 1.92 & 1 \\
\hline d & 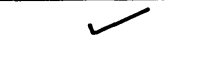 & $\gamma$ & $\sim$ & 1.33 & 1.11 \\
\hline
\end{tabular}

Figure 5.16: Comparison of proposed fatigue damage approach and Miner's rule with experimental fatigue life data of low carbon steel 
It can be seen from the figure that experimental data are from high cycle zone and observe data shows initially conservative and then non-conservative lives and maximum variation is 1.33 as shown in Figure 5.17.

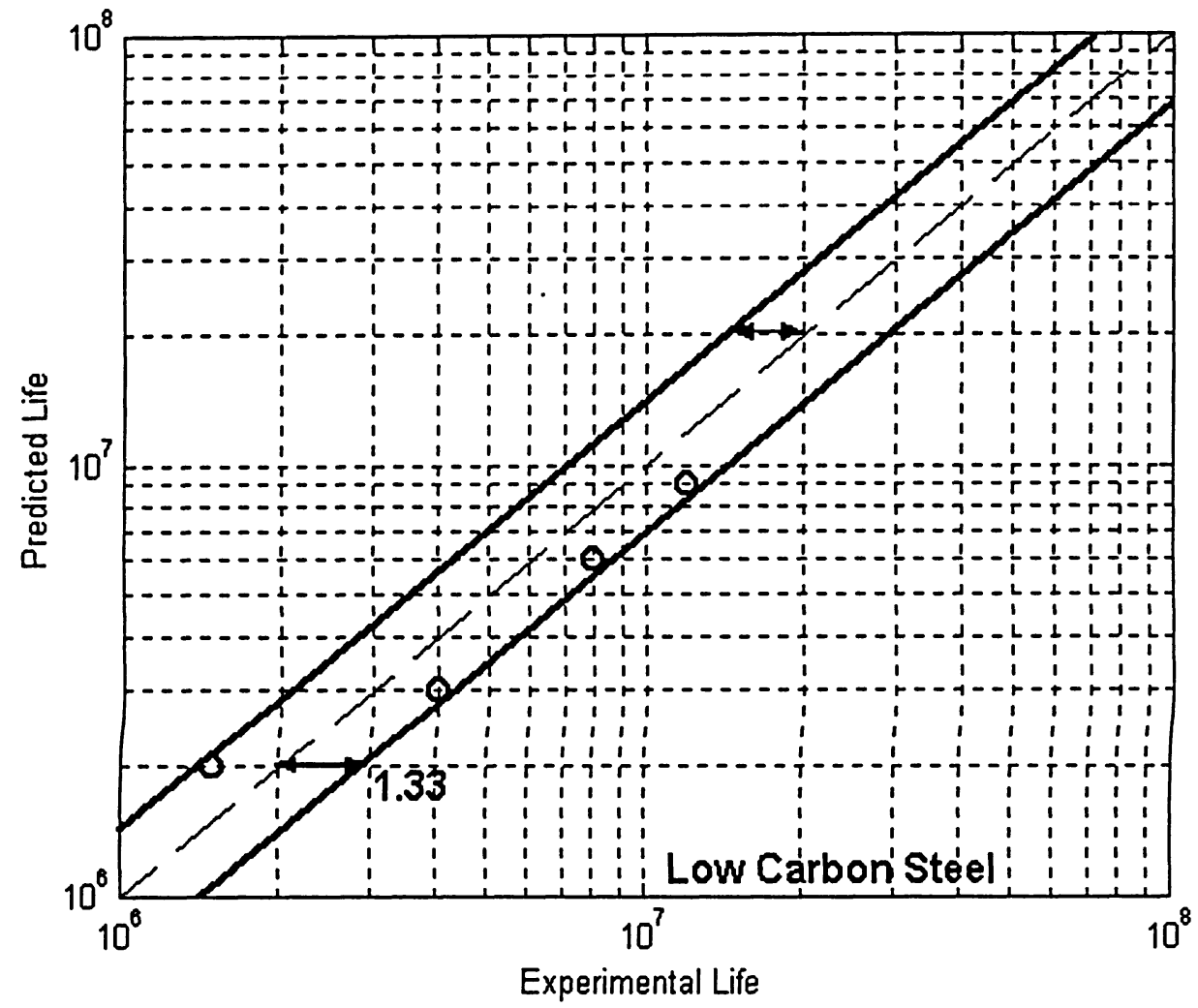

Figure 5.17 Experimental and calculated lives data for low carbon steel. 


\section{CHAPTER SIX}

\section{Discussion}

Fatigue analysis using the concept of a critical plane of maximum shear strain is very effective because the critical plane concept is based on the fracture mode or initiation mechanism of cracks. In the critical plane concept, after determining the maximum shear strain plane, Brown and Miller [33] defined fatigue parameter as combination of normal and shear strain. Stulen and Cummings [34] defined fatigue parameter as combination of normal and shear stress. Fatemi and Socie [35] presented fatigue parameter as combination of normal stress and shear strain. Critical plane defined by Liu's parameter [49], on the other hand, is associated with two different physical modes of failure and the parameter consists of Mode I and Mode II energy components. Liu's parameter does not account for the effect of mean stress but it predicts fatigue life better, regardless of temperature, materials, and load ratio. Chu et al. [50] formulated normal and shear energy components based on the Smith-Waston-Topper parameter. They determined the critical plane and the largest damage parameter from the transformation of strains and stress onto planes spaced at equal increments using a generalized Mroz model. This parameter is based on the maximum value of the damage parameter rather than being defined on planes of maximum stress or strain. Glinka et al. [51] proposed a multiaxial fatigue life parameter based on the summation of the product of normal and shear strains and stresses on the critical plane, which is assumed to be the plane of maximum shear strain. The parameters discussed here are defined on specific planes and state of stress through combination of normal and shear strain and stress ranges. These parameters depend upon the choice of the critical plane and the stress and strain ranges acting on that plane. 
Varvani's approach is used as basis to develop a new approach for variable amplitude loading in this thesis. In this approach, critical plane is defined by the largest strain and stress Mohr's circles during loading and unloading. This approach does not require any empirical fitting factor; it takes care of mean stress and additional hardening, phenomenological factors such as sequence loading, memory effect, and small cycles. The effect due to sequence loading is studied for variable loading. It is found that the loading sequence has a great influence in the stress-strain response of hysteresis loops. Memory effect concept has been used to close hysteresis loops in each block loading history. The small amplitude cycles corresponds to $50 \%$ to $100 \%$ of the endurance limit are also studied. Results indicate that small cycles exceeding $50 \%$ of the fatigue endurance limit contribute to the accumulated damage.

The present proposed analysis considers the evaluation of different materials of low carbon steel, 2024T351, and 7075-T761 aluminum alloys. It is found that steel under variable loading has shown better results than aluminum alloys However, the overall results verifies that fatigue life prediction of different materials fall within an acceptable limits.

Macha [36] has introduced sign effect, which supports the sign changes during unloading and reloading. It is noticed that the negative and positive signs are provided in the bracket, in such a way that the values will always remain positive inside the bracket. In case of loading, the value will be added and in case of unloading, the value will be deducted. The negative value outside the bracket takes care of negative sign (during unloading cycle). Hence, in loading, unloading and reloading Ramberg-Osgood equations, the sign effect proposed by Macha was already incorporated as a built-in feature.

It can be observed from Figures 5.2, 5.7, 5.10, 5.13 and 5.16 that life prediction values agree with experimental life data as Case ' $a$ ' to Case ' $d$ ' by introducing sequence effect, memory effect and small cycle effect gradually. Hence it can be deduced that introducing 
phenomenological factors in the study has provided a favorable results for assessing fatigue lives of components under variable loading conditions.

A comparison of the predicted lives with experimental lives for a number of materials and variable loading conditions showed that life data to be skewed from the line of comparison with conservative at longer lives and non-conservative at shorter lives. Figures (6.1.3- 6.5.3) compared the results of damage analysis in this thesis with the widely accepted Miner's rule and available experimental data in the literature. The proposed parameter successfully correlated uniaxial fatigue lives within a factor that varied with materials from 1.5 to a maximum value of 3.0. The poorest correlation of factor 3.0, in fatigue life of 2024-T351 aluminum alloy may be due to such factors as environmental effect, heat treatment impact, geometry of fatigue specimens and finally human errors while carrying out experiments. Other materials like low carbon steel and 7075-T761 showed good correlation of life data and the proposed fatigue parameter. 


\section{CHAPTER SEVEN}

\section{Conclusions and Recommendations}

\subsection{Conclusions:}

Many fatigue theories developed after 1980 were examined from the viewpoints of: empirical formulas, modifications of coffin-Manson equation, application of stress or strain invariant, use of space averages of stress or strain invariants, critical plane approaches, use of energy and finally energy based-critical plane damage approaches. Considering all the above-mentioned methods, this thesis attempts to further extend Varvani's fatigue damage approach for variable amplitude and uniaxial loading condition. This approach integrates the normal energy range and the shear energy range calculated for the critical plane on which the stress and strain Mohr's circles are largest during peaks and valleys of block loading histories. The normal and shear energies in this parameter have been weighted by the tensile and shear fatigue properties. In this thesis further phenomenological factor extends the applicability of Varvani's equation to be applied for variable amplitude loading conditions. Through the study carried out, the following conclusions can be drawn:

1. An algorithm for calculating fatigue damage for uniaxial loading is proposed which uses Varvani's approach as a foundation and takes care of phenomenological factors such as sequence effect, memory effect and small cycle effect.

2. It is found that the loading sequence has a great influence in the stress-strain response of hysteresis loops. Memory effect concept has been used to close hysteresis loops in each block loading history. The small amplitude cycles of 50\% 
to $100 \%$ of the endurance limit were also studied. Results indicate that small cycles exceeding $50 \%$ of the fatigue endurance limit contributes to the accumulated fatigue damage. The effect of small cycles has also been considered on fatigue damage analysis procedure.

3. Fatigue life predictions based on proposed approach for both CAL and VAL were found in good agreement with experimental fatigue life values of different materials extracted from literatures.

\subsection{Recommendations:}

For many components the most frequent cause of failure is fatigue phenomenon. This problem is very important, and for more than 100 years investigators have sought to alleviate and quantify the effects of fatigue damage. Estimation of fatigue life of a material subjected to variable amplitude loading needs a suitable algorithm and damage analysis methodology. This study has put an effort to develop such algorithm for uniaxial variable amplitude loading by taking Varvani's approach as a basis. The following points need further study to extend the applicability of the proposed model. Due to the rarely available experimental data in the literature, further experimental investigations are required to more accurately evaluate the fatigue damage under variable amplitude loading conditions. This fatigue damage approach is developed for a uniaxial state but it can be extended for multiaxial random loading by considering some more parameters. It is also worthwhile to conduct multiaxial fatigue tests under variable amplitude loading conditions where most engineering structures experience such loading complexity. Availability of such experimental data is required to evaluate and extend the damage model for variable multiaxial loading conditions using the proposed damage approach. 


\section{REFERENCES}

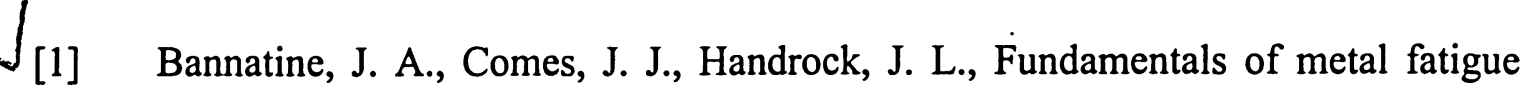
analysis, Prentice-Hall,Inc., 1990.

[2] Grifffith A. A., Philos. Trans. R. Soc. London, A221, 1920, p. 163-165.

[3] Irwin G. R., Fracturing of Metals, American Society for Metals, Cleveland, Ohio, 20, 1949, p.147.

[4] Irwin G. R., "Analysis of Stresses and Strains near the End of a Crack Traversing a Plate," Trans. ASME, J. Appl. Mech., E24, 1957, p.361-364.

[5] Palmgren A., "Durability of ball Bearings," ZVDI, 68, No. 14, 1924, p. 339-341 (in German).

[6] Miner M. A., "Cumulative damage in fatigue, " J. Appl. Mech.,12, Trans. ASME, 67, 1945, p. A159-A164.

[7] Marco, S. M. and Starkey, W. L., A concept of fatigue damages. Transaction of the ASME, 1954, 76, p. 627-632.

[8] Kommers, J. B., The effect of overstress in fatigue on the endurance life of steel, ASTM, 1945, 45, p. 532-541.

[9] Bennett, J. A., A study of damaging effect of fatigue stressing on X4130 steel. Processings, $A S M E$, 1946, 46, p. 693-714.

[10] Splitzer, R.and Corten, H.T., Effect of loading sequence on cumulative fatigue damage of 7071-T6 aluminum alloy. ASTM, 1961, 61, p. 719-731.

[11] Freudenthal, A.M. and Heller, R.A., On the stress interaction in fatigue and a cumulative damage rule, Journal of the Aerospace Science, 1959, 26(7), p. 431442.

[12] Manson, S.S, Nachigall, A.J. and Freche, J. C., A proposed new relation for cumulative fatigue damage in bending, ASTM, 1961, 61, p. 679-703.

[13] Marrow, J. D., The effect of selected sub-cycle sequences in fatigue loading histories, ASME Publication, 1986, 72, p. 43-60.

[14] Grover, H.J., An observation concerning the cycle ratio in cumulative damage. ASTM, PA, 1960, 5, p. 120-124. 
[15] Mason, S.S., Interface between fatigue, creep and fracture. International Journal of Fracture Mechanics, 1966, 2, p. 328-363.

[16] Mason, S.S. and Halford, G. R., Practical implementation of the double linear damage rule and damage rule and damage curve approach for treating cumulative fatigue damage, International Journal of fatigue, 1981, 17(2), p. 169-192.

[17] Mason, S.S. and Halford, G. R., complexities of high-temperature metal fatigue: some steps toward understanding. Israel Journal of Technology, 1983, 21, p. 29 53.

[18] Bui-Quoc-T., An interaction effect consideration in cumulative damage on a mild steel under torsion loading. Proceedings of the $5^{\text {th }}$ International Conference on Fracture, 1981, 5, p. 2625-2633.

[19] Wheeler, O E., Spectrum loading and crack growth. ASME, 1972, D94 (1), p. 181-186.

[20] Willenburg, J., Eagle, R. M. and Wood, H. A., A crack growth retardation model using an effective stress concept, AFFDL TM-71-1-FBR, 1971,14, p. 67-75.

[21] Miller, K. J. and Zachariah, K. P., Cumulative damage laws for fatigue crack initiation and stage I propagation. Journal of Strain Analysis, 1977, 12(4), p. 262270.

[22] Ibrahim, M. F.E and Miller, K. J., Determination of fatigue cracks initiation life. Fatigue of Engineering Material and Structure, 1980, 2, p. 351-360.

[23] Miller, K J, Mohamed, H J and de los Rios, E R., Fatigue damage accumulation above and below the fatigue limit, Mechanical Engineering Publication, 2, 1986, p. 491-511.

[24.] Garud, Y.S.,Proc.Symp., On Methods for predicting materials life in fatigue, ASME, 1979,103, p.247-251.

[25] Golos, K. and Ellyin, F., A total strain energy density theory for cumulative fatigue damage. ASME Journal of Pressure Vessel Technology, 1988, 110, p.3641.

[26] Ellyin, F., cyclic strain energy density as a criterion for multiaxial fatigue failure. Mechanical Engineering Publications, Suffolk, UK, 1989, 89, p. 571-583.

[27] Ellyin, F. and Golos, K., Plastic strain energy in fatigue failure. Trans. ASME, J. Engng Mater. Technol. 1988, 110, p.63-69. 
[28] Lachowicz, C T., Calculation of the elastic-plastic strain energy density under cyclic and random loading. International Journal of fatigue, 2000, 23, p. 643-652.

[29] Tachankov, D S and Vesselinov, K V., Fatigue life prediction under random loading using total hysteresis energy, I J of Pressure Vessels and Piping, 1998, 75, p.955-960.

[30] Kachanov, K M. and Rabotnov, Y. N., Time to the rupture process under creep condition, Izvestiia, $A N S S S R$, 1984, (8), p. 26-31.

[31] Chaboche, J L., Fracture mechanics and damage mechanics: complementarily of approaches. In Numerical Method in fracture Mechanics Fourth International Conference, ed.,Swansea, 1987, p. 309-324.

[32] Chow, C L and Wei, Y A., Model of continuum damage mechanics for fatigue failure. International Journal of fatigue, 1991, 50, p. 301-316.

[33] Brown, M.W. and Miller, K.J.,Proc., Conf. Of the fatigue of metals, the institute of mechanical Engineerings, 46, 1973, p.746-752.

[34] Stulen, F.B. and Cummings, H.N., Proc. ASME, 1954, 54, p.822-827.

[35] Fatemi, A. and Socie, D.F., Fatigue fracture of engineering materials and structures, 1988, 11, p. 149-154.

[36] Lagoda, $\mathrm{T}$ and Macha, E., Energy approach to fatigue life estimation under combined tensile with torsion, VII Summer School of fracture Mechanics, 2001, 6, p.18-22.

[37] Lagoda, T., Energy model for fatigue life estimates under uniaxial random loading I, International journal of fatigue, 2001, 23, p.467-480.

[38] Lagoda, T., Energy model for fatigue life estimate under uniaxial random loading II: Verification of model, International journal of fatigue, 2001, 21, p.481-489.

[39] Varvani-Farahani, A., A new critical plane parameter for fatigue life assessment of various metallic materials subjected to in-phase and out-of-phase multiaxial fatigue loading conditions, International journal of fatigue, 2000, 22, p.295-305.

[40] Kilman, V., Fatigue life prediction for a material under programmable loading using the cyclic stress-strain properties. Material Science and Engineering, 1983, $68, \mathrm{p} .1-10$.

[41] Everett, R. A., The effect of load sequencing on the fatigue life of 2024-T3 aluminum alloy. International journal of fatigue, 1997, 19, p.289-293. 
[42] Dowling, N. E. and Wilson W. K., Notch member fatigue life prediction by local strain approach, Westinghouse research labs, the American Society of Automotive Engineer, 1977, 32, p.55-106.

[43] Pompetzki, M. A. and Topper, T. H., Effect of compressive underloads and tensile overloads on fatigue damage accumulation in 2024-T351 aluminum, $J$ Testing Eval, JTEVA, 1990; 18(1), p. 53-61.

[44] Naigu, C. Sequence effect of small amplitude cycles on fatigue crack initiation and propagation in 2024-T351 aluminum, International journal of fatigue, 2001, 23, p.807-815.

[45] Elber, W., Fatigue crack closure. J Engng Fracture Mechanics. 1970; 2(1), p. $37-$ 45.

[46] Fatemi A, Socie DF. A critical plane approach to multiaxial fatigue damage including out of phase loading. Fatigue Fract. Eng Mater Struct 1988; 11, p. 4965.

[47] Agerskov, H., fatigue in steel structures under random loading. Journal of Constructional Steel Research, 2000, 53, p. 283-305.

[48] Wu.W.F, Liou.H.Y and Tsse.H.C., Estimation of fatigue damage and fatigue life of components under random loading.

[49] Song, Y., Fatigue test and analysis of concrete under biaxial cyclic load. Journal of Constructional concrete Research, 1999, 51, p. 223-235.

[50] Chu, C.C.,Conle, F.A., and Bonnen J.F., Multiaxial stress-strain modeling and fatigue life prediction of SAE axle shaft, ASTM STP 1191, 1993, 30, 37-54.

[51] Glinka, G., Shen, G., Plumtree, A., A multiaxial fatigue strain energy density parameter related to the critical plane. Fatigue Fract Eng Mater Struct 1995, 18, $37-46$. 


\section{Appendix A}

(a) Fatigue properties of Low Carbon Steel

Cyclic Strength Coefficient

Cyclic Strain hardening exponent

Fatigue Strength Coefficient

Fatigue Strength Exponent

Fatigue Ductility Coefficient

Fatigue Ductility Exponent

Modulus of Elasticity

$$
\begin{aligned}
& \mathrm{K}^{\prime}=549.5 \mathrm{MPa} \\
& \mathrm{n}^{\prime}=0.193 \\
& \sigma_{{ }_{\mathrm{f}}}=842 \mathrm{MPa} \\
& \mathrm{b}=-0.102 \\
& \boldsymbol{\varepsilon}_{\mathrm{f}_{\mathrm{f}}}^{\prime}=0.204 \\
& \mathrm{c}=-0.499 \\
& \mathrm{E}=204 \mathrm{GPa}
\end{aligned}
$$

\begin{tabular}{|c|c|c|c|c|}
\hline 277.18 & 270.53 & 202.90 & 131.88 & 79.13 \\
\hline-277.18 & -270.53 & -202.90 & -131.88 & -79.13 \\
\hline 464.58 & 453.43 & 340.07 & 221.05 & 132.63 \\
\hline-464.58 & -453.43 & -340.07 & -221.05 & -132.63 \\
\hline 277.18 & 270.53 & 202.90 & 131.88 & 79.13 \\
\hline-277.18 & -270.53 & -202.90 & -131.88 & -79.13 \\
\hline 441.15 & 430.56 & 322.92 & 209.90 & 125.94 \\
\hline-441.15 & -430.56 & -322.92 & -209.90 & -125.94 \\
\hline 441.15 & 430.56 & 322.92 & 209.90 & 125.94 \\
\hline-441.15 & -430.56 & -322.92 & -209.90 & -125.94 \\
\hline 488.00 & 476.29 & 357.22 & 232.19 & 139.31 \\
\hline-488.00 & -476.29 & -357.22 & -232.19 & -139.31 \\
\hline 277.18 & 270.53 & 202.90 & 131.88 & 79.13 \\
\hline-277.18 & -270.53 & -202.90 & -131.88 & -79.13 \\
\hline 347.46 & 339.12 & 254.34 & 165.32 & 99.19 \\
\hline-347.46 & -339.12 & -254.34 & -165.32 & -99.19 \\
\hline 488.00 & 476.29 & 357.22 & 232.19 & 139.31 \\
\hline-488.00 & -476.29 & -357.22 & -232.19 & -139.31 \\
\hline 417.73 & 407.70 & 305.78 & 198.76 & 119.25 \\
\hline-417.73 & -407.70 & -305.78 & -198.76 & -119.25 \\
\hline 488.00 & 476.29 & 357.22 & 232.19 & 139.31 \\
\hline-488.00 & -476.29 & -357.22 & -232.19 & -139.31 \\
\hline 347.46 & 339.12 & 254.34 & 165.32 & 99.19 \\
\hline-347.46 & -339.12 & -254.34 & -165.32 & -99.19 \\
\hline 441.15 & 430.56 & 322.92 & 209.90 & 125.94 \\
\hline-441.15 & -430.56 & -322.92 & -209.90 & -125.94 \\
\hline 277.18 & 270.53 & 202.90 & 131.88 & 79.13 \\
\hline-277.18 & -270.53 & -202.90 & -131.88 & -79.13 \\
\hline 488.00 & 476.29 & 357.22 & 232.19 & 139.31 \\
\hline-488.00 & -476.29 & -357.22 & -232.19 & -139.31 \\
\hline 464.58 & 453.43 & 340.07 & 221.05 & 132.63 \\
\hline
\end{tabular}

Peak and valley stress data (in $\mathrm{MPa}$ ) of low carbon steel reported by Kilman [40] 


\begin{tabular}{|c|c|c|c|c|}
\hline-464.58 & -453.43 & -340.07 & -221.05 & -132.63 \\
\hline 300.61 & 293.39 & 220.05 & 143.03 & 85.82 \\
\hline-300.61 & -293.39 & -220.05 & -143.03 & -85.82 \\
\hline 277.18 & 270.53 & 202.90 & 131.88 & 79.13 \\
\hline-277.18 & -270.53 & -202.90 & -131.88 & -79.13 \\
\hline 300.61 & 293.39 & 220.05 & 143.03 & 85.82 \\
\hline-300.61 & -293.39 & -220.05 & -143.03 & -85.82 \\
\hline 441.15 & 430.56 & 322.92 & 209.90 & 125.94 \\
\hline-441.15 & -430.56 & -322.92 & -209.90 & -125.94 \\
\hline 488.00 & 476.29 & 357.22 & 232.19 & 139.31 \\
\hline-488.00 & -476.29 & -357.22 & -232.19 & -139.31 \\
\hline 417.73 & 407.70 & 305.78 & 198.76 & 119.25 \\
\hline-417.73 & -407.70 & -305.78 & -198.76 & -119.25 \\
\hline 277.18 & 270.53 & 202.90 & 131.88 & 79.13 \\
\hline-277.18 & -270.53 & -202.90 & -131.88 & -79.13 \\
\hline 488.00 & 476.29 & 357.22 & 232.19 & 139.31 \\
\hline-488.00 & -476.29 & -357.22 & -232.19 & -139.31 \\
\hline 300.61 & 293.39 & 220.05 & 143.03 & 85.82 \\
\hline-300.61 & -293.39 & -220.05 & -143.03 & -85.82 \\
\hline 394.30 & 384.84 & 288.63 & 187.61 & 112.57 \\
\hline-394.30 & -384.84 & -288.63 & -187.61 & -112.57 \\
\hline 324.03 & 316.26 & 237.19 & 154.17 & 92.50 \\
\hline-324.03 & -316.26 & -237.19 & -154.17 & -92.50 \\
\hline 417.73 & 407.70 & 305.78 & 198.76 & 119.25 \\
\hline-417.73 & -407.70 & -305.78 & -198.76 & -119.25 \\
\hline 417.73 & 407.70 & 305.78 & 198.76 & 119.25 \\
\hline-417.73 & -407.70 & -305.78 & -198.76 & -119.25 \\
\hline 300.61 & 293.39 & 220.05 & 143.03 & 85.82 \\
\hline 300.61 & 293.39 & 220.05 & 143.03 & 85.82 \\
\hline 394.30 & 384.84 & 288.63 & 187.61 & 112.57 \\
\hline-394.30 & -384.84 & -288.63 & -187.61 & -112.57 \\
\hline 277.18 & 270.53 & 202.90 & 131.88 & 79.13 \\
\hline-277.18 & -270.53 & -202.90 & -131.88 & -79.13 \\
\hline 394.30 & 384.84 & 288.63 & 187.61 & 112.57 \\
\hline-394.30 & -384.84 & -288.63 & -187.61 & -112.57 \\
\hline 417.73 & 407.70 & 305.78 & 198.76 & 119.25 \\
\hline-417.73 & -407.70 & -305.78 & -198.76 & -119.25 \\
\hline 488.00 & 476.29 & 357.22 & 232.19 & 139.31 \\
\hline-488.00 & -476.29 & -357.22 & -232.19 & -139.31 \\
\hline 394.30 & 384.84 & 288.63 & 187.61 & 112.57 \\
\hline-394.30 & -384.84 & -288.63 & -187.61 & -112.57 \\
\hline 324.03 & 316.26 & 237.19 & 154.17 & 92.50 \\
\hline-324.03 & -316.26 & -237.19 & -154.17 & -92.50 \\
\hline 277.18 & 270.53 & 202.90 & 131.88 & 79.13 \\
\hline-277.18 & -270.53 & -202.90 & -131.88 & -79.13 \\
\hline 277.18 & 270.53 & 202.90 & 131.88 & 79.13 \\
\hline-277.18 & -270.53 & -202.90 & -131.88 & -79.13 \\
\hline 277.18 & 270.53 & 202.90 & 131.88 & 79.13 \\
\hline
\end{tabular}




\begin{tabular}{|c|c|c|c|c|}
\hline-277.18 & -270.53 & -202.90 & -131.88 & -79.13 \\
\hline 417.73 & 407.70 & 305.78 & 198.76 & 119.25 \\
\hline-417.73 & -407.70 & -305.78 & -198.76 & -119.25 \\
\hline 464.58 & 453.43 & 340.07 & 221.05 & 132.63 \\
\hline-464.58 & -453.43 & -340.07 & -221.05 & -132.63 \\
\hline 464.58 & 453.43 & 340.07 & 221.05 & 132.63 \\
\hline-464.58 & -453.43 & -340.07 & -221.05 & -132.63 \\
\hline 417.73 & 407.70 & 305.78 & 198.76 & 119.25 \\
\hline-417.73 & -407.70 & -305.78 & -198.76 & -119.25 \\
\hline 464.58 & 453.43 & 340.07 & 221.05 & 132.63 \\
\hline-464.58 & -453.43 & -340.07 & -221.05 & -132.63 \\
\hline 347.46 & 339.12 & 254.34 & 165.32 & 99.19 \\
\hline-347.46 & -339.12 & -254.34 & -165.32 & -99.19 \\
\hline 347.46 & 339.12 & 254.34 & 165.32 & 99.19 \\
\hline-347.46 & -339.12 & -254.34 & -165.32 & -99.19 \\
\hline 488.00 & 476.29 & 357.22 & 232.19 & 139.31 \\
\hline-488.00 & -476.29 & -357.22 & -232.19 & -139.31 \\
\hline 277.18 & 270.53 & 202.90 & 131.88 & 79.13 \\
\hline-277.18 & -270.53 & -202.90 & -131.88 & -79.13 \\
\hline 370.88 & 361.98 & 271.48 & 176.46 & 105.88 \\
\hline-370.88 & -361.98 & -271.48 & -176.46 & -105.88 \\
\hline 464.58 & 453.43 & 340.07 & 221.05 & 132.63 \\
\hline-464.58 & -453.43 & -340.07 & -221.05 & -132.63 \\
\hline 347.46 & 339.12 & 254.34 & 165.32 & 99.19 \\
\hline-347.46 & -339.12 & -254.34 & -165.32 & -99.19 \\
\hline 488.00 & 476.29 & 357.22 & 232.19 & 139.31 \\
\hline-488.00 & -476.29 & -357.22 & -232.19 & -139.31 \\
\hline 370.88 & 361.98 & 271.48 & 176.46 & 105.88 \\
\hline-370.88 & -361.98 & -271.48 & -176.46 & -105.88 \\
\hline 464.58 & 453.43 & 340.07 & 221.05 & 132.63 \\
\hline-464.58 & -453.43 & -340.07 & -221.05 & -132.63 \\
\hline 488.00 & 476.29 & 357.22 & 232.19 & 139.31 \\
\hline-488.00 & -476.29 & -357.22 & -232.19 & -139.31 \\
\hline 347.46 & 339.12 & 254.34 & 165.32 & 99.19 \\
\hline-347.46 & -339.12 & -254.34 & -165.32 & -99.19 \\
\hline 394.30 & 384.84 & 288.63 & 187.61 & 112.57 \\
\hline-394.30 & -384.84 & -288.63 & -187.61 & -112.57 \\
\hline 464.58 & 453.43 & 340.07 & 221.05 & 132.63 \\
\hline-464.58 & -453.43 & -340.07 & -221.05 & -132.63 \\
\hline 417.73 & 407.70 & 305.78 & 198.76 & 119.25 \\
\hline-417.73 & -407.70 & -305.78 & -198.76 & -119.25 \\
\hline 277.18 & 270.53 & 202.90 & 131.88 & 79.13 \\
\hline-277.18 & -270.53 & -202.90 & -131.88 & -79.13 \\
\hline 370.88 & 361.98 & 271.48 & 176.46 & 105.88 \\
\hline-370.88 & -361.98 & -271.48 & -176.46 & -105.88 \\
\hline 324.03 & 316.26 & 237.19 & 154.17 & 92.50 \\
\hline-324.03 & -316.26 & -237.19 & -154.17 & -92.50 \\
\hline 488.00 & 476.29 & 357.22 & 232.19 & 139.31 \\
\hline
\end{tabular}




\begin{tabular}{|c|c|c|c|c|}
\hline-488.00 & -476.29 & -357.22 & -232.19 & -139.31 \\
\hline 464.58 & 453.43 & 340.07 & 221.05 & 132.63 \\
\hline-464.58 & -453.43 & -340.07 & -221.05 & -132.63 \\
\hline 488.00 & 476.29 & 357.22 & 232.19 & 139.31 \\
\hline-488.00 & -476.29 & -357.22 & -232.19 & -139.31 \\
\hline 324.03 & 316.26 & 237.19 & 154.17 & 92.50 \\
\hline-324.03 & -316.26 & -237.19 & -154.17 & -92.50 \\
\hline 324.03 & 316.26 & 237.19 & 154.17 & 92.50 \\
\hline-324.03 & -316.26 & -237.19 & -154.17 & -92.50 \\
\hline 394.30 & 384.84 & 288.63 & 187.61 & 112.57 \\
\hline-394.30 & -384.84 & -288.63 & -187.61 & -112.57 \\
\hline 324.03 & 316.26 & 237.19 & 154.17 & 92.50 \\
\hline-324.03 & -316.26 & -237.19 & -154.17 & -92.50 \\
\hline 277.18 & 270.53 & 202.90 & 131.88 & 79.13 \\
\hline-277.18 & -270.53 & -202.90 & -131.88 & -79.13 \\
\hline 300.61 & 293.39 & 220.05 & 143.03 & 85.82 \\
\hline-300.61 & -293.39 & -220.05 & -143.03 & -85.82 \\
\hline 394.30 & 384.84 & 288.63 & 187.61 & 112.57 \\
\hline-394.30 & -384.84 & -288.63 & -187.61 & -112.57 \\
\hline 324.03 & 316.26 & 237.19 & 154.17 & 92.50 \\
\hline-324.03 & -316.26 & -237.19 & -154.17 & -92.50 \\
\hline 488.00 & 476.29 & 357.22 & 232.19 & 139.31 \\
\hline-488.00 & -476.29 & -357.22 & -232.19 & -139.31 \\
\hline 277.18 & 270.53 & 202.90 & 131.88 & 79.13 \\
\hline-277.18 & -270.53 & -202.90 & -131.88 & -79.13 \\
\hline 488.00 & 476.29 & 357.22 & 232.19 & 139.31 \\
\hline-488.00 & -476.29 & -357.22 & -232.19 & -139.31 \\
\hline 464.58 & 453.43 & 340.07 & 221.05 & 132.63 \\
\hline-464.58 & -453.43 & -340.07 & -221.05 & -132.63 \\
\hline 347.46 & 339.12 & 254.34 & 165.32 & 99.19 \\
\hline-347.46 & -339.12 & -254.34 & -165.32 & -99.19 \\
\hline 300.61 & 293.39 & 220.05 & 143.03 & 85.82 \\
\hline-300.61 & -293.39 & -220.05 & -143.03 & -85.82 \\
\hline 394.30 & 384.84 & 288.63 & 187.61 & 112.57 \\
\hline-394.30 & -384.84 & -288.63 & -187.61 & -112.57 \\
\hline 488.00 & 476.29 & 357.22 & 232.19 & 139.31 \\
\hline-488.00 & -476.29 & -357.22 & -232.19 & -139.31 \\
\hline 394.30 & 384.84 & 288.63 & 187.61 & 112.57 \\
\hline-394.30 & -384.84 & -288.63 & -187.61 & -112.57 \\
\hline 324.03 & 316.26 & 237.19 & 154.17 & 92.50 \\
\hline-324.03 & -316.26 & -237.19 & -154.17 & -92.50 \\
\hline 300.61 & 293.39 & 220.05 & 143.03 & 85.82 \\
\hline-300.61 & -293.39 & -220.05 & -143.03 & -85.82 \\
\hline 417.73 & 407.70 & 305.78 & 198.76 & 119.25 \\
\hline-417.73 & -407.70 & -305.78 & -198.76 & -119.25 \\
\hline 347.46 & 339.12 & 254.34 & 165.32 & 99.19 \\
\hline-347.46 & -339.12 & -254.34 & -165.32 & -99.19 \\
\hline 347.46 & 339.12 & 254.34 & 165.32 & 99.19 \\
\hline
\end{tabular}




\begin{tabular}{|c|c|c|c|c|}
\hline-347.46 & -339.12 & -254.34 & -165.32 & -99.19 \\
\hline 324.03 & 316.26 & 237.19 & 154.17 & 92.50 \\
\hline-324.03 & -316.26 & -237.19 & -154.17 & -92.50 \\
\hline 394.30 & 384.84 & 288.63 & 187.61 & 112.57 \\
\hline-394.30 & -384.84 & -288.63 & -187.61 & -112.57 \\
\hline 488.00 & 476.29 & 357.22 & 232.19 & 139.31 \\
\hline-488.00 & -476.29 & -357.22 & -232.19 & -139.31 \\
\hline 277.18 & 270.53 & 202.90 & 131.88 & 79.13 \\
\hline-277.18 & -270.53 & -202.90 & -131.88 & -79.13 \\
\hline 300.61 & 293.39 & 220.05 & 143.03 & 85.82 \\
\hline-300.61 & -293.39 & -220.05 & -143.03 & -85.82 \\
\hline 324.03 & 316.26 & 237.19 & 154.17 & 92.50 \\
\hline-324.03 & -316.26 & -237.19 & -154.17 & -92.50 \\
\hline 370.88 & 361.98 & 271.48 & 176.46 & 105.88 \\
\hline-370.88 & -361.98 & -271.48 & -176.46 & -105.88 \\
\hline 394.30 & 384.84 & 288.63 & 187.61 & 112.57 \\
\hline-394.30 & -384.84 & -288.63 & -187.61 & -112.57 \\
\hline 300.61 & 293.39 & 220.05 & 143.03 & 85.82 \\
\hline-300.61 & -293.39 & -220.05 & -143.03 & -85.82 \\
\hline 488.00 & 476.29 & 357.22 & 232.19 & 139.31 \\
\hline-488.00 & -476.29 & -357.22 & -232.19 & -139.31 \\
\hline 488.00 & 476.29 & 357.22 & 232.19 & 139.31 \\
\hline-488.00 & -476.29 & -357.22 & -232.19 & -139.31 \\
\hline 324.03 & 316.26 & 237.19 & 154.17 & 92.50 \\
\hline-324.03 & -316.26 & -237.19 & -154.17 & -92.50 \\
\hline 488.00 & 476.29 & 357.22 & 232.19 & 139.31 \\
\hline-488.00 & -476.29 & -357.22 & -232.19 & -139.31 \\
\hline 394.30 & 384.84 & 288.63 & 187.61 & 112.57 \\
\hline-394.30 & -384.84 & -288.63 & -187.61 & -112.57 \\
\hline 277.18 & 270.53 & 202.90 & 131.88 & 79.13 \\
\hline-277.18 & -270.53 & -202.90 & -131.88 & -79.13 \\
\hline 417.73 & 407.70 & 305.78 & 198.76 & 119.25 \\
\hline-417.73 & -407.70 & -305.78 & -198.76 & -119.25 \\
\hline 417.73 & 407.70 & 305.78 & 198.76 & 119.25 \\
\hline-417.73 & -407.70 & -305.78 & -198.76 & -119.25 \\
\hline 488.00 & 476.29 & 357.22 & 232.19 & 139.31 \\
\hline-488.00 & -476.29 & -357.22 & -232.19 & -139.31 \\
\hline 324.03 & 316.26 & 237.19 & 154.17 & 92.50 \\
\hline-324.03 & -316.26 & -237.19 & -154.17 & -92.50 \\
\hline 277.18 & 270.53 & 202.90 & 131.88 & 79.13 \\
\hline-277.18 & -270.53 & -202.90 & -131.88 & -79.13 \\
\hline 347.46 & 339.12 & 254.34 & 165.32 & 99.19 \\
\hline-347.46 & -339.12 & -254.34 & -165.32 & -99.19 \\
\hline 464.58 & 453.43 & 340.07 & 221.05 & 132.63 \\
\hline-464.58 & -453.43 & -340.07 & -221.05 & -132.63 \\
\hline 324.03 & 316.26 & 237.19 & 154.17 & 92.50 \\
\hline-324.03 & -316.26 & -237.19 & -154.17 & -92.50 \\
\hline 277.18 & 270.53 & 202.90 & 131.88 & 79.13 \\
\hline
\end{tabular}




\begin{tabular}{|c|c|c|c|c|}
\hline-277.18 & -270.53 & -202.90 & -131.88 & -79.13 \\
\hline 370.88 & 361.98 & 271.48 & 176.46 & 105.88 \\
\hline-370.88 & -361.98 & -271.48 & -176.46 & -105.88 \\
\hline
\end{tabular}


(b) Fatigue properties of 7075-T761 Aluminum alloy

Cyclic Strength Coefficient

Cyclic Strain hardening exponent

Fatigue Strength Coefficient

$\mathrm{K}^{\prime}=852 \mathrm{MPa}$

Fatigue Strength Exponent

n' $=0.074$

$\sigma_{\mathrm{f}}^{\prime}=1231 \mathrm{MPa}$

$\mathrm{b}=-0.122$

Fatigue Ductility Coefficient

$\varepsilon_{\mathrm{f}}^{\prime}=0.263$

$\mathrm{c}=-0.806$

Fatigue Ductility Exponent

$\mathrm{E}=700 \mathrm{GPa}$

Modulus of Elasticity

Peak and valley stress data (in MPa) of 7075-T761 Aluminum alloy reported by Wu [49]

\begin{tabular}{|c|c|c|c|c|}
\hline 42.88 & 41.36 & 30.57 & 28.77 & 26.98 \\
\hline-42.88 & -41.36 & -30.57 & -28.77 & -26.98 \\
\hline 85.76 & 82.72 & 61.14 & 57.55 & 53.95 \\
\hline-85.76 & -82.72 & -61.14 & -57.55 & -53.95 \\
\hline 215.74 & 208.10 & 153.81 & 144.77 & 135.72 \\
\hline-215.74 & -208.10 & -153.81 & -144.77 & -135.72 \\
\hline 108.54 & 104.70 & 77.39 & 72.83 & 68.28 \\
\hline-108.54 & -104.70 & -77.39 & -72.83 & -68.28 \\
\hline 93.8 & 90.48 & 66.88 & 62.94 & 59.01 \\
\hline-93.8 & -90.48 & -66.88 & -62.94 & -59.01 \\
\hline 259.96 & 250.76 & 185.34 & 174.44 & 163.54 \\
\hline-259.96 & -250.76 & -185.34 & -174.44 & -163.54 \\
\hline 93.8 & 90.48 & 66.88 & 62.94 & 59.01 \\
\hline-93.8 & -90.48 & -66.88 & -62.94 & -59.01 \\
\hline 85.76 & 82.72 & 61.14 & 57.55 & 53.95 \\
\hline-85.76 & -82.72 & -61.14 & -57.55 & -53.95 \\
\hline 151.42 & 146.06 & 107.96 & 101.61 & 95.26 \\
\hline-151.42 & -146.06 & -107.96 & -101.61 & -95.26 \\
\hline 108.54 & 104.70 & 77.39 & 72.83 & 68.28 \\
\hline-108.54 & -104.70 & -77.39 & -72.83 & -68.28 \\
\hline 108.54 & 104.70 & 77.39 & 72.83 & 68.28 \\
\hline-108.54 & -104.70 & -77.39 & -72.83 & -68.28 \\
\hline 93.8 & 90.48 & 66.88 & 62.94 & 59.01 \\
\hline-93.8 & -90.48 & -66.88 & -62.94 & -59.01 \\
\hline 85.76 & 82.72 & 61.14 & 57.55 & 53.95 \\
\hline-85.76 & -82.72 & -61.14 & -57.55 & -53.95 \\
\hline 151.42 & 146.06 & 107.96 & 101.61 & 95.26 \\
\hline-151.42 & -146.06 & -107.96 & -101.61 & -95.26 \\
\hline 85.76 & 82.72 & 61.14 & 57.55 & 53.95 \\
\hline-85.76 & -82.72 & -61.14 & -57.55 & -53.95 \\
\hline 324.28 & 312.80 & 231.20 & 217.60 & 204.00 \\
\hline-324.28 & -312.80 & -231.20 & -217.60 & -204.00 \\
\hline
\end{tabular}




\begin{tabular}{|c|c|c|c|c|}
\hline 237.18 & 228.78 & 169.10 & 159.15 & 149.21 \\
\hline 237.18 & 228.78 & 169.10 & 159.15 & 149.21 \\
\hline 259.96 & 250.76 & 185.34 & 174.44 & 163.54 \\
\hline-259.96 & -250.76 & -185.34 & -174.44 & -163.54 \\
\hline 219.76 & 211.98 & 156.68 & 147.46 & 138.25 \\
\hline-219.76 & -211.98 & -156.68 & -147.46 & -138.25 \\
\hline 108.54 & 104.70 & 77.39 & 72.83 & 68.28 \\
\hline-108.54 & -104.70 & -77.39 & -72.83 & -68.28 \\
\hline 85.76 & 82.72 & 61.14 & 57.55 & 53.95 \\
\hline-85.76 & -82.72 & -61.14 & -57.55 & -53.95 \\
\hline 219.76 & 211.98 & 156.68 & 147.46 & 138.25 \\
\hline-219.76 & -211.98 & -156.68 & -147.46 & -138.25 \\
\hline 108.54 & 104.70 & 77.39 & 72.83 & 68.28 \\
\hline-108.54 & -104.70 & -77.39 & -72.83 & -68.28 \\
\hline 108.54 & 104.70 & 77.39 & 72.83 & 68.28 \\
\hline-108.54 & -104.70 & -77.39 & -72.83 & -68.28 \\
\hline 219.76 & 211.98 & 156.68 & 147.46 & 138.25 \\
\hline-219.76 & -211.98 & -156.68 & -147.46 & -138.25 \\
\hline 64.32 & 62.04 & 45.86 & 43.16 & 40.46 \\
\hline-64.32 & -62.04 & -45.86 & -43.16 & -40.46 \\
\hline 42.88 & 41.36 & 30.57 & 28.77 & 26.98 \\
\hline-42.88 & -41.36 & -30.57 & -28.77 & -26.98 \\
\hline 21.44 & 20.68 & 15.29 & 14.39 & 13.49 \\
\hline-21.44 & -20.68 & -15.29 & -14.39 & -13.49 \\
\hline 64.32 & 62.04 & 45.86 & 43.16 & 40.46 \\
\hline-64.32 & -62.04 & -45.86 & -43.16 & -40.46 \\
\hline 64.32 & 62.04 & 45.86 & 43.16 & 40.46 \\
\hline-64.32 & -62.04 & -45.86 & -43.16 & -40.46 \\
\hline 85.76 & 82.72 & 61.14 & 57.55 & 53.95 \\
\hline-85.76 & -82.72 & -61.14 & -57.55 & -53.95 \\
\hline 237.18 & 228.78 & 169.10 & 159.15 & 149.21 \\
\hline-237.18 & -228.78 & -169.10 & -159.15 & -149.21 \\
\hline 237.18 & 228.78 & 169.10 & 159.15 & 149.21 \\
\hline-237.18 & -228.78 & -169.10 & -159.15 & -149.21 \\
\hline 108.54 & 104.70 & 77.39 & 72.83 & 68.28 \\
\hline-108.54 & -104.70 & -77.39 & -72.83 & -68.28 \\
\hline 129.98 & 125.38 & 92.67 & 87.22 & 81.77 \\
\hline-129.98 & -125.38 & -92.67 & -87.22 & -81.77 \\
\hline 151.42 & 146.06 & 107.96 & 101.61 & 95.26 \\
\hline-151.42 & -146.06 & -107.96 & -101.61 & -95.26 \\
\hline 64.32 & 62.04 & 45.86 & 43.16 & 40.46 \\
\hline-64.32 & -62.04 & -45.86 & -43.16 & -40.46 \\
\hline 172.86 & 166.74 & 123.24 & 115.99 & 108.74 \\
\hline-172.86 & -166.74 & -123.24 & -115.99 & -108.74 \\
\hline 172.86 & 166.74 & 123.24 & 115.99 & 108.74 \\
\hline-172.86 & -166.74 & -123.24 & -115.99 & -108.74 \\
\hline 21.44 & 20.68 & 15.29 & 14.39 & 13.49 \\
\hline-21.44 & -20.68 & -15.29 & -14.39 & -13.49 \\
\hline
\end{tabular}




\begin{tabular}{|c|c|c|c|c|}
\hline 194.3 & 187.42 & 138.53 & 130.38 & 122.23 \\
\hline-194.3 & -187.42 & -138.53 & -130.38 & -122.23 \\
\hline 85.76 & 82.72 & 61.14 & 57.55 & 53.95 \\
\hline-85.76 & -82.72 & -61.14 & -57.55 & -53.95 \\
\hline 21.44 & 20.68 & 15.29 & 14.39 & 13.49 \\
\hline-21.44 & -20.68 & -15.29 & -14.39 & -13.49 \\
\hline 259.96 & 250.76 & 185.34 & 174.44 & 163.54 \\
\hline-259.96 & -250.76 & -185.34 & -174.44 & -163.54 \\
\hline 194.3 & 187.42 & 138.53 & 130.38 & 122.23 \\
\hline-194.3 & -187.42 & -138.53 & -130.38 & -122.23 \\
\hline 21.44 & 20.68 & 15.29 & 14.39 & 13.49 \\
\hline-21.44 & -20.68 & -15.29 & -14.39 & -13.49 \\
\hline 172.86 & 166.74 & 123.24 & 115.99 & 108.74 \\
\hline-172.86 & -166.74 & -123.24 & -115.99 & -108.74 \\
\hline 259.96 & 250.76 & 185.34 & 174.44 & 163.54 \\
\hline-259.96 & -250.76 & -185.34 & -174.44 & -163.54 \\
\hline 259.96 & 250.76 & 185.34 & 174.44 & 163.54 \\
\hline-259.96 & -250.76 & -185.34 & -174.44 & -163.54 \\
\hline 237.18 & 228.78 & 169.10 & 159.15 & 149.21 \\
\hline-237.18 & -228.78 & -169.10 & -159.15 & -149.21 \\
\hline 108.54 & 104.70 & 77.39 & 72.83 & 68.28 \\
\hline-108.54 & -104.70 & -77.39 & -72.83 & -68.28 \\
\hline 108.54 & 104.70 & 77.39 & 72.83 & 68.28 \\
\hline-108.54 & -104.70 & -77.39 & -72.83 & -68.28 \\
\hline 129.98 & 125.38 & 92.67 & 87.22 & 81.77 \\
\hline-129.98 & -125.38 & -92.67 & -87.22 & -81.77 \\
\hline 85.76 & 82.72 & 61.14 & 57.55 & 53.95 \\
\hline-85.76 & -82.72 & -61.14 & -57.55 & -53.95 \\
\hline 324.28 & 312.80 & 231.20 & 217.60 & 204.00 \\
\hline-324.28 & -312.80 & -231.20 & -217.60 & -204.00 \\
\hline
\end{tabular}


(c) Fatigue properties of 2024-T3 aluminum

Cyclic Strength Coefficient

Cyclic Strain hardening exponent

Fatigue Strength Coefficient

Fatigue Strength Exponent

Fatigue Ductility Coefficient

Fatigue Ductility Exponent

Modulus of Elasticity

$$
\begin{aligned}
& \mathrm{K}^{\prime}=852 \mathrm{MPa} \\
& \mathrm{n}^{\prime}=0.074 \\
& \sigma^{\prime}=1231 \mathrm{MPa} \\
& b=-0.122 \\
& \varepsilon^{\prime}=0.263 \\
& \mathrm{c}=-0.806 \\
& \mathrm{E}=700 \mathrm{GPa}
\end{aligned}
$$

\begin{tabular}{|c|c|c|c|}
\hline 4.55 & 4.31 & 4.19 & 3.94 \\
\hline-50.10 & -47.40 & -46.04 & -43.33 \\
\hline-31.88 & -30.16 & -29.30 & -27.58 \\
\hline-27.33 & -25.85 & -25.11 & -23.64 \\
\hline 173.09 & 163.73 & 159.05 & 149.70 \\
\hline 40.99 & 38.78 & 37.67 & 35.45 \\
\hline 141.20 & 133.57 & 129.75 & 122.12 \\
\hline 86.54 & 81.87 & 79.53 & 74.85 \\
\hline 131.40 & 124.30 & 120.75 & 113.65 \\
\hline 73.43 & 69.46 & 67.48 & 63.51 \\
\hline 162.32 & 153.55 & 149.16 & 140.39 \\
\hline 50.24 & 47.53 & 46.17 & 43.45 \\
\hline 143.00 & 135.27 & 131.40 & 123.67 \\
\hline 81.16 & 76.77 & 74.58 & 70.19 \\
\hline 143.00 & 135.27 & 131.40 & 123.67 \\
\hline 85.03 & 80.43 & 78.13 & 73.54 \\
\hline 131.40 & 124.30 & 120.75 & 113.65 \\
\hline 50.24 & 47.53 & 46.17 & 43.45 \\
\hline 143.00 & 135.27 & 131.40 & 123.67 \\
\hline 69.57 & 65.81 & 63.93 & 60.17 \\
\hline 127.54 & 120.65 & 117.20 & 110.30 \\
\hline 81.16 & 76.77 & 74.58 & 70.19 \\
\hline 127.54 & 120.65 & 117.20 & 110.30 \\
\hline 69.57 & 65.81 & 63.93 & 60.17 \\
\hline 139.13 & 131.61 & 127.85 & 120.33 \\
\hline 69.57 & 65.81 & 63.93 & 60.17 \\
\hline 166.19 & 157.20 & 152.71 & 143.73 \\
\hline 69.57 & 65.81 & 63.93 & 60.17 \\
\hline 123.67 & 116.99 & 113.65 & 106.96 \\
\hline 139.13 & 131.61 & 127.85 & 120.33 \\
\hline 69.57 & 65.81 & 63.93 & 60.17 \\
\hline 123.67 & 116.99 & 113.65 & 106.96 \\
\hline 69.57 & 65.81 & 63.93 & 60.17 \\
\hline 139.13 & 131.61 & 127.85 & 120.33 \\
\hline 46.38 & 43.87 & 42.62 & 40.11 \\
\hline 139.13 & 131.61 & 127.85 & 120.33 \\
\hline
\end{tabular}

Peak and valley stress data (in MPa) of 2024-T3 aluminum reported by Everett [41] 


\begin{tabular}{|c|c|c|c|}
\hline 81.16 & 76.77 & 74.58 & 70.19 \\
\hline 123.67 & 116.99 & 113.65 & 106.96 \\
\hline 65.70 & 62.15 & 60.37 & 56.82 \\
\hline 139.13 & 131.61 & 127.85 & 120.33 \\
\hline 65.70 & 62.15 & 60.37 & 56.82 \\
\hline 139.13 & 131.61 & 127.85 & 120.33 \\
\hline 54.11 & 51.18 & 49.72 & 46.80 \\
\hline 127.54 & 120.65 & 117.20 & 110.30 \\
\hline 54.11 & 51.18 & 49.72 & 46.80 \\
\hline 143.00 & 135.27 & 131.40 & 123.67 \\
\hline 85.03 & 80.43 & 78.13 & 73.54 \\
\hline 139.13 & 131.61 & 127.85 & 120.33 \\
\hline 46.38 & 43.87 & 42.62 & 40.11 \\
\hline 139.13 & 131.61 & 127.85 & 120.33 \\
\hline 85.03 & 80.43 & 78.13 & 73.54 \\
\hline 139.13 & 131.61 & 127.85 & 120.33 \\
\hline 69.57 & 65.81 & 63.93 & 60.17 \\
\hline 139.13 & 131.61 & 127.85 & 120.33 \\
\hline 69.57 & 65.81 & 63.93 & 60.17 \\
\hline 139.13 & 131.61 & 127.85 & 120.33 \\
\hline 69.57 & 65.81 & 63.93 & 60.17 \\
\hline 162.32 & 153.55 & 149.16 & 140.39 \\
\hline 69.57 & 65.81 & 63.93 & 60.17 \\
\hline 162.32 & 153.55 & 149.16 & 140.39 \\
\hline 69.57 & 65.81 & 63.93 & 60.17 \\
\hline 131.40 & 124.30 & 120.75 & 113.65 \\
\hline 69.57 & 65.81 & 63.93 & 60.17 \\
\hline 139.13 & 131.61 & 127.85 & 120.33 \\
\hline 69.57 & 65.81 & 63.93 & 60.17 \\
\hline 131.40 & 124.30 & 120.75 & 113.65 \\
\hline 77.30 & 73.12 & 71.03 & 66.85 \\
\hline 139.13 & 131.61 & 127.85 & 120.33 \\
\hline 69.57 & 65.81 & 63.93 & 60.17 \\
\hline 123.67 & 116.99 & 113.65 & 106.96 \\
\hline 85.03 & 80.43 & 78.13 & 73.54 \\
\hline 143.00 & 135.27 & 131.40 & 123.67 \\
\hline 65.70 & 62.15 & 60.37 & 56.82 \\
\hline 123.67 & 116.99 & 113.65 & 106.96 \\
\hline 69.57 & 65.81 & 63.93 & 60.17 \\
\hline 127.54 & 120.65 & 117.20 & 110.30 \\
\hline 73.43 & 69.46 & 67.48 & 63.51 \\
\hline 143.00 & 135.27 & 131.40 & 123.67 \\
\hline 69.57 & 65.81 & 63.93 & 60.17 \\
\hline 123.67 & 116.99 & 113.65 & 106.96 \\
\hline 50.24 & 47.53 & 46.17 & 43.45 \\
\hline 131.40 & 124.30 & 120.75 & 113.65 \\
\hline 85.03 & 80.43 & 78.13 & 73.54 \\
\hline 162.32 & 153.55 & 149.16 & 140.39 \\
\hline
\end{tabular}




\begin{tabular}{|c|c|c|c|}
\hline 85.03 & 80.43 & 78.13 & 73.54 \\
\hline 139.13 & 131.61 & 127.85 & 120.33 \\
\hline 65.70 & 62.15 & 60.37 & 56.82 \\
\hline 123.67 & 116.99 & 113.65 & 106.96 \\
\hline 69.57 & 65.81 & 63.93 & 60.17 \\
\hline 139.13 & 131.61 & 127.85 & 120.33 \\
\hline 69.57 & 65.81 & 63.93 & 60.17 \\
\hline 139.13 & 131.61 & 127.85 & 120.33 \\
\hline 69.57 & 65.81 & 63.93 & 60.17 \\
\hline 177.78 & 168.17 & 163.37 & 153.76 \\
\hline 69.57 & 65.81 & 63.93 & 60.17 \\
\hline 158.46 & 149.89 & 145.61 & 137.04 \\
\hline 69.57 & 65.81 & 63.93 & 60.17 \\
\hline 143.00 & 135.27 & 131.40 & 123.67 \\
\hline 85.03 & 80.43 & 78.13 & 73.54 \\
\hline 139.13 & 131.61 & 127.85 & 120.33 \\
\hline 69.57 & 65.81 & 63.93 & 60.17 \\
\hline 123.67 & 116.99 & 113.65 & 106.96 \\
\hline 69.57 & 65.81 & 63.93 & 60.17 \\
\hline 143.00 & 135.27 & 131.40 & 123.67 \\
\hline 96.62 & 91.40 & 88.79 & 83.56 \\
\hline 143.00 & 135.27 & 131.40 & 123.67 \\
\hline 54.11 & 51.18 & 49.72 & 46.80 \\
\hline 162.32 & 153.55 & 149.16 & 140.39 \\
\hline 54.11 & 51.18 & 49.72 & 46.80 \\
\hline 177.78 & 168.17 & 163.37 & 153.76 \\
\hline 85.03 & 80.43 & 78.13 & 73.54 \\
\hline 158.46 & 149.89 & 145.61 & 137.04 \\
\hline 96.62 & 91.40 & 88.79 & 83.56 \\
\hline 158.46 & 149.89 & 145.61 & 137.04 \\
\hline 81.16 & 76.77 & 74.58 & 70.19 \\
\hline 158.46 & 149.89 & 145.61 & 137.04 \\
\hline 81.16 & 76.77 & 74.58 & 70.19 \\
\hline 158.46 & 149.89 & 145.61 & 137.04 \\
\hline 81.16 & 76.77 & 74.58 & 70.19 \\
\hline 181.65 & 171.83 & 166.92 & 157.10 \\
\hline 81.16 & 76.77 & 74.58 & 70.19 \\
\hline 185.51 & 175.48 & 170.47 & 160.44 \\
\hline 100.49 & 95.05 & 92.34 & 86.91 \\
\hline 158.46 & 149.89 & 145.61 & 137.04 \\
\hline 50.24 & 47.53 & 46.17 & 43.45 \\
\hline 158.46 & 149.89 & 145.61 & 137.04 \\
\hline 81.16 & 76.77 & 74.58 & 70.19 \\
\hline 158.46 & 149.89 & 145.61 & 137.04 \\
\hline 81.16 & 76.77 & 74.58 & 70.19 \\
\hline 158.46 & 149.89 & 145.61 & 137.04 \\
\hline 81.16 & 76.77 & 74.58 & 70.19 \\
\hline 193.24 & 182.80 & 177.57 & 167.13 \\
\hline
\end{tabular}




\begin{tabular}{|c|c|c|c|}
\hline 119.81 & 113.33 & 110.10 & 103.62 \\
\hline 135.27 & 127.96 & 124.30 & 116.99 \\
\hline 81.16 & 76.77 & 74.58 & 70.19 \\
\hline 243.48 & 230.32 & 223.74 & 210.58 \\
\hline 30.92 & 29.25 & 28.41 & 26.74 \\
\hline 200.97 & 190.11 & 184.68 & 173.81 \\
\hline 3.86 & 3.66 & 3.55 & 3.34 \\
\hline 173.92 & 164.52 & 159.82 & 150.41 \\
\hline 100.49 & 95.05 & 92.34 & 86.91 \\
\hline 173.92 & 164.52 & 159.82 & 150.41 \\
\hline 77.30 & 73.12 & 71.03 & 66.85 \\
\hline 173.92 & 164.52 & 159.82 & 150.41 \\
\hline 104.35 & 98.71 & 95.89 & 90.25 \\
\hline 173.92 & 164.52 & 159.82 & 150.41 \\
\hline 119.81 & 113.33 & 110.10 & 103.62 \\
\hline 200.97 & 190.11 & 184.68 & 173.81 \\
\hline 112.08 & 106.02 & 102.99 & 96.93 \\
\hline 282.13 & 266.88 & 259.26 & 244.01 \\
\hline 100.49 & 95.05 & 92.34 & 86.91 \\
\hline 189.38 & 179.14 & 174.02 & 163.78 \\
\hline 100.49 & 95.05 & 92.34 & 86.91 \\
\hline 212.57 & 201.08 & 195.33 & 183.84 \\
\hline 115.94 & 109.68 & 106.54 & 100.28 \\
\hline 177.78 & 168.17 & 163.37 & 153.76 \\
\hline 73.43 & 69.46 & 67.48 & 63.51 \\
\hline 193.24 & 182.80 & 177.57 & 167.13 \\
\hline 73.43 & 69.46 & 67.48 & 63.51 \\
\hline 173.92 & 164.52 & 159.82 & 150.41 \\
\hline 34.78 & 32.90 & 31.96 & 30.08 \\
\hline 173.92 & 164.52 & 159.82 & 150.41 \\
\hline 34.78 & 32.90 & 31.96 & 30.08 \\
\hline 173.92 & 164.52 & 159.82 & 150.41 \\
\hline 115.94 & 109.68 & 106.54 & 100.28 \\
\hline 173.92 & 164.52 & 159.82 & 150.41 \\
\hline 115.94 & 109.68 & 106.54 & 100.28 \\
\hline 173.92 & 164.52 & 159.82 & 150.41 \\
\hline 73.43 & 69.46 & 67.48 & 63.51 \\
\hline 173.92 & 164.52 & 159.82 & 150.41 \\
\hline 100.49 & 95.05 & 92.34 & 86.91 \\
\hline 181.65 & 171.83 & 166.92 & 157.10 \\
\hline 119.81 & 113.33 & 110.10 & 103.62 \\
\hline 173.92 & 164.52 & 159.82 & 150.41 \\
\hline 100.49 & 95.05 & 92.34 & 86.91 \\
\hline 193.24 & 182.80 & 177.57 & 167.13 \\
\hline 100.49 & 95.05 & 92.34 & 86.91 \\
\hline 216.43 & 204.73 & 198.88 & 187.18 \\
\hline 100.49 & 95.05 & 92.34 & 86.91 \\
\hline 177.78 & 168.17 & 163.37 & 153.76 \\
\hline
\end{tabular}




\begin{tabular}{|c|c|c|c|}
\hline 115.94 & 109.68 & 106.54 & 100.28 \\
\hline 177.78 & 168.17 & 163.37 & 153.76 \\
\hline 100.49 & 95.05 & 92.34 & 86.91 \\
\hline 200.97 & 190.11 & 184.68 & 173.81 \\
\hline 100.49 & 95.05 & 92.34 & 86.91 \\
\hline 185.51 & 175.48 & 170.47 & 160.44 \\
\hline 77.30 & 73.12 & 71.03 & 66.85 \\
\hline 193.24 & 182.80 & 177.57 & 167.13 \\
\hline 104.35 & 98.71 & 95.89 & 90.25 \\
\hline 200.97 & 190.11 & 184.68 & 173.81 \\
\hline 77.30 & 73.12 & 71.03 & 66.85 \\
\hline 197.11 & 186.45 & 181.12 & 170.47 \\
\hline 77.30 & 73.12 & 71.03 & 66.85 \\
\hline 139.13 & 131.61 & 127.85 & 120.33 \\
\hline 69.57 & 65.81 & 63.93 & 60.17 \\
\hline 123.67 & 116.99 & 113.65 & 106.96 \\
\hline 69.57 & 65.81 & 63.93 & 60.17 \\
\hline 143.00 & 135.27 & 131.40 & 123.67 \\
\hline 96.62 & 91.40 & 88.79 & 83.56 \\
\hline 143.00 & 135.27 & 131.40 & 123.67 \\
\hline 54.11 & 51.18 & 49.72 & 46.80 \\
\hline 162.32 & 153.55 & 149.16 & 140.39 \\
\hline 54.11 & 51.18 & 49.72 & 46.80 \\
\hline 177.78 & 168.17 & 163.37 & 153.76 \\
\hline 85.03 & 80.43 & 78.13 & 73.54 \\
\hline 158.46 & 149.89 & 145.61 & 137.04 \\
\hline 96.62 & 91.40 & 88.79 & 83.56 \\
\hline 158.46 & 149.89 & 145.61 & 137.04 \\
\hline 81.16 & 76.77 & 74.58 & 70.19 \\
\hline 158.46 & 149.89 & 145.61 & 137.04 \\
\hline 81.16 & 76.77 & 74.58 & 70.19 \\
\hline 158.46 & 149.89 & 145.61 & 137.04 \\
\hline 81.16 & 76.77 & 74.58 & 70.19 \\
\hline 181.65 & 171.83 & 166.92 & 157.10 \\
\hline 81.16 & 76.77 & 74.58 & 70.19 \\
\hline 185.51 & 175.48 & 170.47 & 160.44 \\
\hline 100.49 & 95.05 & 92.34 & 86.91 \\
\hline 158.46 & 149.89 & 145.61 & 137.04 \\
\hline 50.24 & 47.53 & 46.17 & 43.45 \\
\hline 158.46 & 149.89 & 145.61 & 137.04 \\
\hline 81.16 & 76.77 & 74.58 & 70.19 \\
\hline 158.46 & 149.89 & 145.61 & 137.04 \\
\hline 81.16 & 76.77 & 74.58 & 70.19 \\
\hline 158.46 & 149.89 & 145.61 & 137.04 \\
\hline 81.16 & 76.77 & 74.58 & 70.19 \\
\hline 193.24 & 182.80 & 177.57 & 167.13 \\
\hline 119.81 & 113.33 & 110.10 & 103.62 \\
\hline 135.27 & 127.96 & 124.30 & 116.99 \\
\hline
\end{tabular}




\begin{tabular}{|c|c|c|c|}
\hline 81.16 & 76.77 & 74.58 & 70.19 \\
\hline 243.48 & 230.32 & 223.74 & 210.58 \\
\hline 30.92 & 29.25 & 28.41 & 26.74 \\
\hline 200.97 & 190.11 & 184.68 & 173.81 \\
\hline 3.86 & 3.66 & 3.55 & 3.34 \\
\hline 173.92 & 164.52 & 159.82 & 150.41 \\
\hline 100.49 & 95.05 & 92.34 & 86.91 \\
\hline 173.92 & 164.52 & 159.82 & 150.41 \\
\hline 77.30 & 73.12 & 71.03 & 66.85 \\
\hline 173.92 & 164.52 & 159.82 & 150.41 \\
\hline 104.35 & 98.71 & 95.89 & 90.25 \\
\hline 173.92 & 164.52 & 159.82 & 150.41 \\
\hline 119.81 & 113.33 & 110.10 & 103.62 \\
\hline 200.97 & 190.11 & 184.68 & 173.81 \\
\hline 112.08 & 106.02 & 102.99 & 96.93 \\
\hline 251.21 & 237.63 & 230.84 & 217.27 \\
\hline 100.49 & 95.05 & 92.34 & 86.91 \\
\hline 189.38 & 179.14 & 174.02 & 163.78 \\
\hline 100.49 & 95.05 & 92.34 & 86.91 \\
\hline 212.57 & 201.08 & 195.33 & 183.84 \\
\hline 115.94 & 109.68 & 106.54 & 100.28 \\
\hline 177.78 & 168.17 & 163.37 & 153.76 \\
\hline 73.43 & 69.46 & 67.48 & 63.51 \\
\hline 193.24 & 182.80 & 177.57 & 167.13 \\
\hline 73.43 & 69.46 & 67.48 & 63.51 \\
\hline 173.92 & 164.52 & 159.82 & 150.41 \\
\hline 34.78 & 32.90 & 31.96 & 30.08 \\
\hline 173.92 & 164.52 & 159.82 & 150.41 \\
\hline 34.78 & 32.90 & 31.96 & 30.08 \\
\hline 173.92 & 164.52 & 159.82 & 150.41 \\
\hline 115.94 & 109.68 & 106.54 & 100.28 \\
\hline 173.92 & 164.52 & 159.82 & 150.41 \\
\hline 115.94 & 109.68 & 106.54 & 100.28 \\
\hline 173.92 & 164.52 & 159.82 & 150.41 \\
\hline 73.43 & 69.46 & 67.48 & 63.51 \\
\hline 173.92 & 164.52 & 159.82 & 150.41 \\
\hline 100.49 & 95.05 & 92.34 & 86.91 \\
\hline 181.65 & 171.83 & 166.92 & 157.10 \\
\hline 119.81 & 113.33 & 110.10 & 103.62 \\
\hline 173.92 & 164.52 & 159.82 & 150.41 \\
\hline 100.49 & 95.05 & 92.34 & 86.91 \\
\hline 193.24 & 182.80 & 177.57 & 167.13 \\
\hline 100.49 & 95.05 & 92.34 & 86.91 \\
\hline 216.43 & 204.73 & 198.88 & 187.18 \\
\hline 100.49 & 95.05 & 92.34 & 86.91 \\
\hline 177.78 & 168.17 & 163.37 & 153.76 \\
\hline 115.94 & 109.68 & 106.54 & 100.28 \\
\hline 177.78 & 168.17 & 163.37 & 153.76 \\
\hline
\end{tabular}




\begin{tabular}{|c|c|c|c|}
\hline 100.49 & 95.05 & 92.34 & 86.91 \\
\hline 200.97 & 190.11 & 184.68 & 173.81 \\
\hline 100.49 & 95.05 & 92.34 & 86.91 \\
\hline 185.51 & 175.48 & 170.47 & 160.44 \\
\hline 77.30 & 73.12 & 71.03 & 66.85 \\
\hline 193.24 & 182.80 & 177.57 & 167.13 \\
\hline 104.35 & 98.71 & 95.89 & 90.25 \\
\hline 200.97 & 190.11 & 184.68 & 173.81 \\
\hline 77.30 & 73.12 & 71.03 & 66.85 \\
\hline 197.11 & 186.45 & 181.12 & 170.47 \\
\hline 77.30 & 73.12 & 71.03 & 66.85 \\
\hline 173.92 & 164.52 & 159.82 & 150.41 \\
\hline 73.43 & 69.46 & 67.48 & 63.51 \\
\hline 173.92 & 164.52 & 159.82 & 150.41 \\
\hline 100.49 & 95.05 & 92.34 & $86.91^{\circ}$ \\
\hline 181.65 & 171.83 & 166.92 & 157.10 \\
\hline 119.81 & 113.33 & 110.10 & 103.62 \\
\hline 173.92 & 164.52 & 159.82 & 150.41 \\
\hline 100.49 & 95.05 & 92.34 & 86.91 \\
\hline 193.24 & 182.80 & 177.57 & 167.13 \\
\hline 100.49 & 95.05 & 92.34 & 86.91 \\
\hline 216.43 & 204.73 & 198.88 & 187.18 \\
\hline 100.49 & 95.05 & 92.34 & 86.91 \\
\hline 177.78 & 168.17 & 163.37 & 153.76 \\
\hline 115.94 & 109.68 & 106.54 & 100.28 \\
\hline 177.78 & 168.17 & 163.37 & 153.76 \\
\hline 100.49 & 95.05 & 92.34 & 86.91 \\
\hline 200.97 & 190.11 & 184.68 & 173.81 \\
\hline 100.49 & 95.05 & 92.34 & 86.91 \\
\hline 185.51 & 175.48 & 170.47 & 160.44 \\
\hline 77.30 & 73.12 & 71.03 & 66.85 \\
\hline 193.24 & 182.80 & 177.57 & 167.13 \\
\hline 104.35 & 98.71 & 95.89 & 90.25 \\
\hline 200.97 & 190.11 & 184.68 & 173.81 \\
\hline 77.30 & 73.12 & 71.03 & 66.85 \\
\hline 197.11 & 186.45 & 181.12 & 170.47 \\
\hline 38.65 & 36.56 & 35.51 & 33.43 \\
\hline 139.13 & 131.61 & 127.85 & 120.33 \\
\hline-3.86 & -3.66 & -3.55 & -3.34 \\
\hline 123.67 & 116.99 & 113.65 & 106.96 \\
\hline-38.65 & -36.56 & -35.51 & -33.43 \\
\hline 143.00 & 135.27 & 131.40 & 123.67 \\
\hline 96.62 & 91.40 & 88.79 & 83.56 \\
\hline 143.00 & 135.27 & 131.40 & 123.67 \\
\hline 54.11 & 51.18 & 49.72 & 46.80 \\
\hline 200.97 & 190.11 & 184.68 & 173.81 \\
\hline 54.11 & 51.18 & 49.72 & 46.80 \\
\hline 200.97 & 190.11 & 184.68 & 173.81 \\
\hline
\end{tabular}




\begin{tabular}{|c|c|c|c|}
\hline 85.03 & 80.43 & 78.13 & 73.54 \\
\hline 235.75 & 223.01 & 216.64 & 203.90 \\
\hline 96.62 & 91.40 & 88.79 & 83.56 \\
\hline 158.46 & 149.89 & 145.61 & 137.04 \\
\hline 81.16 & 76.77 & 74.58 & 70.19 \\
\hline 158.46 & 149.89 & 145.61 & 137.04 \\
\hline 81.16 & 76.77 & 74.58 & 70.19 \\
\hline 158.46 & 149.89 & 145.61 & 137.04 \\
\hline 81.16 & 76.77 & 74.58 & 70.19 \\
\hline 181.65 & 171.83 & 166.92 & 157.10 \\
\hline 81.16 & 76.77 & 74.58 & 70.19 \\
\hline 185.51 & 175.48 & 170.47 & 160.44 \\
\hline 100.49 & 95.05 & 92.34 & 86.91 \\
\hline 158.46 & 149.89 & 145.61 & 137.04 \\
\hline 50.24 & 47.53 & 46.17 & 43.45 \\
\hline 158.46 & 149.89 & 145.61 & 137.04 \\
\hline 81.16 & 76.77 & 74.58 & 70.19 \\
\hline 158.46 & 149.89 & 145.61 & 137.04 \\
\hline 81.16 & 76.77 & 74.58 & 70.19 \\
\hline 158.46 & 149.89 & 145.61 & 137.04 \\
\hline 81.16 & 76.77 & 74.58 & 70.19 \\
\hline 193.24 & 182.80 & 177.57 & 167.13 \\
\hline 119.81 & 113.33 & 110.10 & 103.62 \\
\hline 135.27 & 127.96 & 124.30 & 116.99 \\
\hline 81.16 & 76.77 & 74.58 & 70.19 \\
\hline 243.48 & 230.32 & 223.74 & 210.58 \\
\hline 30.92 & 29.25 & 28.41 & 26.74 \\
\hline 200.97 & 190.11 & 184.68 & 173.81 \\
\hline 3.86 & 3.66 & 3.55 & 3.34 \\
\hline 173.92 & 164.52 & 159.82 & 150.41 \\
\hline 100.49 & 95.05 & 92.34 & 86.91 \\
\hline 173.92 & 164.52 & 159.82 & 150.41 \\
\hline 77.30 & 73.12 & 71.03 & 66.85 \\
\hline 173.92 & 164.52 & 159.82 & 150.41 \\
\hline 104.35 & 98.71 & 95.89 & 90.25 \\
\hline 173.92 & 164.52 & 159.82 & 150.41 \\
\hline 119.81 & 113.33 & 110.10 & 103.62 \\
\hline 200.97 & 190.11 & 184.68 & 173.81 \\
\hline 112.08 & 106.02 & 102.99 & 96.93 \\
\hline 282.13 & 266.88 & 259.26 & 244.01 \\
\hline 100.49 & 95.05 & 92.34 & 86.91 \\
\hline 189.38 & 179.14 & 174.02 & 163.78 \\
\hline 100.49 & 95.05 & 92.34 & 86.91 \\
\hline 212.57 & 201.08 & 195.33 & 183.84 \\
\hline 115.94 & 109.68 & 106.54 & 100.28 \\
\hline 177.78 & 168.17 & 163.37 & 153.76 \\
\hline 73.43 & 69.46 & 67.48 & 63.51 \\
\hline 193.24 & 182.80 & 177.57 & 167.13 \\
\hline
\end{tabular}




\begin{tabular}{|c|c|c|c|}
\hline 73.43 & 69.46 & 67.48 & 63.51 \\
\hline 173.92 & 164.52 & 159.82 & 150.41 \\
\hline 34.78 & 32.90 & 31.96 & 30.08 \\
\hline 173.92 & 164.52 & 159.82 & 150.41 \\
\hline 34.78 & 32.90 & 31.96 & 30.08 \\
\hline 173.92 & 164.52 & 159.82 & 150.41 \\
\hline 115.94 & 109.68 & 106.54 & 100.28 \\
\hline 173.92 & 164.52 & 159.82 & 150.41 \\
\hline 115.94 & 109.68 & 106.54 & 100.28 \\
\hline 173.92 & 164.52 & 159.82 & 150.41 \\
\hline 73.43 & 69.46 & 67.48 & 63.51 \\
\hline 173.92 & 164.52 & 159.82 & 150.41 \\
\hline 100.49 & 95.05 & 92.34 & 86.91 \\
\hline 181.65 & 171.83 & 166.92 & 157.10 \\
\hline 119.81 & 113.33 & 110.10 & 103.62 \\
\hline 173.92 & 164.52 & 159.82 & 150.41 \\
\hline 100.49 & 95.05 & 92.34 & 86.91 \\
\hline 193.24 & 182.80 & 177.57 & 167.13 \\
\hline 100.49 & 95.05 & 92.34 & 86.91 \\
\hline 216.43 & 204.73 & 198.88 & 187.18 \\
\hline 100.49 & 95.05 & 92.34 & 86.91 \\
\hline 177.78 & 168.17 & 163.37 & 153.76 \\
\hline 115.94 & 109.68 & 106.54 & 100.28 \\
\hline 177.78 & 168.17 & 163.37 & 153.76 \\
\hline 100.49 & 95.05 & 92.34 & 86.91 \\
\hline 200.97 & 190.11 & 184.68 & 173.81 \\
\hline 100.49 & 95.05 & 92.34 & 86.91 \\
\hline 185.51 & 175.48 & 170.47 & 160.44 \\
\hline 127.54 & 120.65 & 117.20 & 110.30 \\
\hline 81.16 & 76.77 & 74.58 & 70.19 \\
\hline 127.54 & 120.65 & 117.20 & 110.30 \\
\hline 69.57 & 65.81 & 63.93 & 60.17 \\
\hline 139.13 & 131.61 & 127.85 & 120.33 \\
\hline 69.57 & 65.81 & 63.93 & 60.17 \\
\hline 166.19 & 157.20 & 152.71 & 143.73 \\
\hline 69.57 & 65.81 & 63.93 & 60.17 \\
\hline 123.67 & 116.99 & 113.65 & 106.96 \\
\hline 139.13 & 131.61 & 127.85 & 120.33 \\
\hline 69.57 & 65.81 & 63.93 & 60.17 \\
\hline 123.67 & 116.99 & 113.65 & 106.96 \\
\hline 69.57 & 65.81 & 63.93 & 60.17 \\
\hline 139.13 & 131.61 & 127.85 & 120.33 \\
\hline 46.38 & 43.87 & 42.62 & 40.11 \\
\hline 139.13 & 131.61 & 127.85 & 120.33 \\
\hline 81.16 & 76.77 & 74.58 & 70.19 \\
\hline 123.67 & 116.99 & 113.65 & 106.96 \\
\hline 65.70 & 62.15 & 60.37 & 56.82 \\
\hline 139.13 & 131.61 & 127.85 & 120.33 \\
\hline
\end{tabular}




\begin{tabular}{|c|c|c|c|}
\hline 65.70 & 62.15 & 60.37 & 56.82 \\
\hline 139.13 & 131.61 & 127.85 & 120.33 \\
\hline 54.11 & 51.18 & 49.72 & 46.80 \\
\hline 127.54 & 120.65 & 117.20 & 110.30 \\
\hline 54.11 & 51.18 & 49.72 & 46.80 \\
\hline 143.00 & 135.27 & 131.40 & 123.67 \\
\hline 85.03 & 80.43 & 78.13 & 73.54 \\
\hline 139.13 & 131.61 & 127.85 & 120.33 \\
\hline 46.38 & 43.87 & 42.62 & 40.11 \\
\hline 139.13 & 131.61 & 127.85 & 120.33 \\
\hline 85.03 & 80.43 & 78.13 & 73.54 \\
\hline 139.13 & 131.61 & 127.85 & 120.33 \\
\hline 69.57 & 65.81 & 63.93 & 60.17 \\
\hline 139.13 & 131.61 & 127.85 & 120.33 \\
\hline 69.57 & 65.81 & 63.93 & 60.17 \\
\hline 139.13 & 131.61 & 127.85 & 120.33 \\
\hline 69.57 & 65.81 & 63.93 & 60.17 \\
\hline 162.32 & 153.55 & 149.16 & 140.39 \\
\hline 69.57 & 65.81 & 63.93 & 60.17 \\
\hline 162.32 & 153.55 & 149.16 & 140.39 \\
\hline 69.57 & 65.81 & 63.93 & 60.17 \\
\hline 131.40 & 124.30 & 120.75 & 113.65 \\
\hline 69.57 & 65.81 & 63.93 & 60.17 \\
\hline 139.13 & 131.61 & 127.85 & 120.33 \\
\hline 69.57 & 65.81 & 63.93 & 60.17 \\
\hline 131.40 & 124.30 & 120.75 & 113.65 \\
\hline 77.30 & 73.12 & 71.03 & 66.85 \\
\hline 139.13 & 131.61 & 127.85 & 120.33 \\
\hline 69.57 & 65.81 & 63.93 & 60.17 \\
\hline 123.67 & 116.99 & 113.65 & 106.96 \\
\hline 85.03 & 80.43 & 78.13 & 73.54 \\
\hline 143.00 & 135.27 & 131.40 & 123.67 \\
\hline 65.70 & 62.15 & 60.37 & 56.82 \\
\hline 123.67 & 116.99 & 113.65 & 106.96 \\
\hline 69.57 & 65.81 & 63.93 & 60.17 \\
\hline 127.54 & 120.65 & 117.20 & 110.30 \\
\hline 73.43 & 69.46 & 67.48 & 63.51 \\
\hline 143.00 & 135.27 & 131.40 & 123.67 \\
\hline 69.57 & 65.81 & 63.93 & 60.17 \\
\hline 123.67 & 116.99 & 113.65 & 106.96 \\
\hline 50.24 & 47.53 & 46.17 & 43.45 \\
\hline 131.40 & 124.30 & 120.75 & 113.65 \\
\hline 85.03 & 80.43 & 78.13 & 73.54 \\
\hline 162.32 & 153.55 & 149.16 & 140.39 \\
\hline 85.03 & 80.43 & 78.13 & 73.54 \\
\hline 139.13 & 131.61 & 127.85 & 120.33 \\
\hline 65.70 & 62.15 & 60.37 & 56.82 \\
\hline 123.67 & 116.99 & 113.65 & 106.96 \\
\hline
\end{tabular}




\begin{tabular}{|c|c|c|c|}
\hline 69.57 & 65.81 & 63.93 & 60.17 \\
\hline 139.13 & 131.61 & 127.85 & 120.33 \\
\hline 69.57 & 65.81 & 63.93 & 60.17 \\
\hline 139.13 & 131.61 & 127.85 & 120.33 \\
\hline 69.57 & 65.81 & 63.93 & 60.17 \\
\hline
\end{tabular}


(d) Fatigue properties of Low carbon steel

Cyclic Strength Coefficient

Cyclic Strain hardening exponent

Fatigue Strength Coefficient

$\mathrm{K}^{\prime}=549.5 \mathrm{MPa}$

Fatigue Strength Exponent

$\mathrm{n}^{\prime}=0.193$

$\sigma_{\mathrm{f}}=842 \mathrm{MPa}$

$\mathrm{b}=-0.102$

Fatigue Ductility Coefficient

$\varepsilon^{\prime}=0.204$

Fatigue Ductility Exponent

$\mathrm{c}=-0.499$

Modulus of Elasticity

$\mathrm{E}=204 \mathrm{GPa}$

Peak and valley stress data (in $\mathrm{MPa}$ ) of low carbon steel reported by Agerskov [47]

\begin{tabular}{|c|c|c|c|c|}
\hline .92 & 2.09 & 1.67 & 1.5 & 1.33 \\
\hline-5.75 & -6.27 & -5.00 & -4.5 & -4.00 \\
\hline 46.00 & 50.18 & 40.00 & 36 & 32.00 \\
\hline-17.25 & -18.82 & -15.00 & -13.5 & -12.00 \\
\hline 86.25 & 94.09 & 75.00 & 67.5 & 60.00 \\
\hline-23.00 & -25.09 & -20.00 & -18 & -16.00 \\
\hline 51.75 & 56.45 & 45.00 & 40.5 & 36.00 \\
\hline-1.92 & -2.09 & -1.67 & -1.5 & -1.33 \\
\hline 74.75 & 81.55 & 65.00 & 58.5 & 52.00 \\
\hline 23.00 & 25.09 & 20.00 & 18 & 16.00 \\
\hline 74.75 & 81.55 & 65.00 & 58.5 & 52.00 \\
\hline 23.00 & 25.09 & 20.00 & 18 & 16.00 \\
\hline 74.75 & 81.55 & 65.00 & 58.5 & 52.00 \\
\hline 11.50 & 12.55 & 10.00 & 9 & 8.00 \\
\hline 51.75 & 56.45 & 45.00 & 40.5 & 36.00 \\
\hline-1.92 & -2.09 & -1.67 & -1.5 & -1.33 \\
\hline 11.50 & 12.55 & 10.00 & 9 & 8.00 \\
\hline 1.92 & 2.09 & 1.67 & 1.5 & 1.33 \\
\hline 11.50 & 12.55 & 10.00 & 9 & 8.00 \\
\hline 103.50 & 112.91 & 90.00 & 81 & 72.00 \\
\hline-17.25 & -18.82 & -15.00 & -13.5 & -12.00 \\
\hline 132.25 & 144.27 & 115.00 & 103.5 & 92.00 \\
\hline-11.50 & -12.55 & -10.00 & -9 & -8.00 \\
\hline 103.50 & 112.91 & 90.00 & 81 & 72.00 \\
\hline 1.92 & 2.09 & 1.67 & 1.5 & 1.33 \\
\hline 57.50 & 62.73 & 50.00 & 45 & 40.00 \\
\hline 51.75 & 56.45 & 45.00 & 40.5 & 36.00 \\
\hline 57.50 & 62.73 & 50.00 & 45 & 40.00 \\
\hline-1.92 & -2.09 & -1.67 & -1.5 & -1.33 \\
\hline 74.75 & 81.55 & 65.00 & 58.5 & 52.00 \\
\hline-1.92 & -2.09 & -1.67 & -1.5 & -1.33 \\
\hline 80.50 & 87.82 & 70.00 & 63 & 56.00 \\
\hline-1.92 & -2.09 & -1.67 & -1.5 & -1.33 \\
\hline 86.25 & 94.09 & 75.00 & 67.5 & 60.00 \\
\hline
\end{tabular}




\begin{tabular}{|c|c|c|c|c|}
\hline-1.92 & -2.09 & -1.67 & -1.5 & -1.33 \\
\hline 80.50 & 87.82 & 70.00 & 63 & 56.00 \\
\hline-11.50 & -12.55 & -10.00 & -9 & -8.00 \\
\hline 28.75 & 31.36 & 25.00 & 22.5 & 20.00 \\
\hline-11.50 & -12.55 & -10.00 & -9 & -8.00 \\
\hline 17.25 & 18.82 & 15.00 & 13.5 & 12.00 \\
\hline-1.92 & -2.09 & -1.67 & -1.5 & -1.33 \\
\hline 34.50 & 37.64 & 30.00 & 27 & 24.00 \\
\hline-5.75 & -6.27 & -5.00 & -4.5 & -4.00 \\
\hline 57.50 & 62.73 & 50.00 & 45 & 40.00 \\
\hline 5.75 & 6.27 & 5.00 & 4.5 & 4.00 \\
\hline 51.75 & 56.45 & 45.00 & 40.5 & 36.00 \\
\hline-23.00 & -25.09 & -20.00 & -18 & -16.00 \\
\hline 57.50 & 62.73 & 50.00 & 45 & 40.00 \\
\hline-11.50 & -12.55 & -10.00 & -9 & -8.00 \\
\hline 69.00 & 75.27 & 60.00 & 54 & 48.00 \\
\hline-11.50 & -12.55 & -10.00 & -9 & -8.00 \\
\hline 23.00 & 25.09 & 20.00 & 18 & 16.00 \\
\hline 11.50 & 12.55 & 10.00 & 9 & 8.00 \\
\hline 28.75 & 31.36 & 25.00 & 22.5 & 20.00 \\
\hline-5.75 & -6.27 & -5.00 & -4.5 & -4.00 \\
\hline 23.00 & 25.09 & 20.00 & 18 & 16.00 \\
\hline-5.75 & -6.27 & -5.00 & -4.5 & -4.00 \\
\hline 34.50 & 37.64 & 30.00 & 27 & 24.00 \\
\hline 28.75 & 31.36 & 25.00 & 22.5 & 20.00 \\
\hline 57.50 & 62.73 & 50.00 & 45 & 40.00 \\
\hline-23.00 & -25.09 & -20.00 & -18 & -16.00 \\
\hline 46.00 & 50.18 & 40.00 & 36 & 32.00 \\
\hline-5.75 & -6.27 & -5.00 & -4.5 & -4.00 \\
\hline 80.50 & 87.82 & 70.00 & 63 & 56.00 \\
\hline 11.50 & 12.55 & 10.00 & 9 & 8.00 \\
\hline 149.50 & 163.09 & 130.00 & 117 & 104.00 \\
\hline-34.50 & -37.64 & -30.00 & -27 & -24.00 \\
\hline 132.25 & 144.27 & 115.00 & 103.5 & 92.00 \\
\hline-11.50 & -12.55 & -10.00 & -9 & -8.00 \\
\hline 40.25 & 43.91 & 35.00 & 31.5 & 28.00 \\
\hline 1.92 & 2.09 & 1.67 & 1.5 & 1.33 \\
\hline 63.25 & 69.00 & 55.00 & 49.5 & 44.00 \\
\hline-11.50 & -12.55 & -10.00 & -9 & -8.00 \\
\hline 63.25 & 69.00 & 55.00 & 49.5 & 44.00 \\
\hline-11.50 & -12.55 & -10.00 & -9 & -8.00 \\
\hline 97.75 & 106.64 & 85.00 & 76.5 & 68.00 \\
\hline-28.75 & -31.36 & -25.00 & -22.5 & -20.00 \\
\hline 40.25 & 43.91 & 35.00 & 31.5 & 28.00 \\
\hline-11.50 & -12.55 & -10.00 & -9 & -8.00 \\
\hline 17.25 & 18.82 & 15.00 & 13.5 & 12.00 \\
\hline 1.92 & 2.09 & 1.67 & 1.5 & 1.33 \\
\hline 11.50 & 12.55 & 10.00 & 9 & 8.00 \\
\hline
\end{tabular}




\begin{tabular}{|c|c|c|c|c|}
\hline-5.75 & -6.27 & -5.00 & -4.5 & -4.00 \\
\hline 63.25 & 69.00 & 55.00 & 49.5 & 44.00 \\
\hline-5.75 & -6.27 & -5.00 & -4.5 & -4.00 \\
\hline 74.75 & 81.55 & 65.00 & 58.5 & 52.00 \\
\hline-11.50 & -12.55 & -10.00 & -9 & -8.00 \\
\hline 40.25 & 43.91 & 35.00 & 31.5 & 28.00 \\
\hline-5.75 & -6.27 & -5.00 & -4.5 & -4.00 \\
\hline 109.25 & 119.18 & 95.00 & 85.5 & 76.00 \\
\hline-17.25 & -18.82 & -15.00 & -13.5 & -12.00 \\
\hline 218.50 & 238.36 & 190.00 & 171 & 152.00 \\
\hline 155.25 & 169.36 & 135.00 & 121.5 & 108.00 \\
\hline 161.00 & 175.64 & 140.00 & 126 & 112.00 \\
\hline-5.75 & -6.27 & -5.00 & -4.5 & -4.00 \\
\hline 166.75 & 181.91 & 145.00 & 130.5 & 116.00 \\
\hline-5.75 & -6.27 & -5.00 & -4.5 & -4.00 \\
\hline 155.25 & 169.36 & 135.00 & 121.5 & 108.00 \\
\hline 11.50 & 12.55 & 10.00 & 9 & 8.00 \\
\hline 109.25 & 119.18 & 95.00 & 85.5 & 76.00 \\
\hline-23.00 & -25.09 & -20.00 & -18 & -16.00 \\
\hline 138.00 & 150.55 & 120.00 & 108 & 96.00 \\
\hline-11.50 & -12.55 & -10.00 & -9 & -8.00 \\
\hline 63.25 & 69.00 & 55.00 & 49.5 & 44.00 \\
\hline 5.75 & 6.27 & 5.00 & 4.5 & 4.00 \\
\hline 40.25 & 43.91 & 35.00 & 31.5 & 28.00 \\
\hline-11.50 & -12.55 & -10.00 & -9 & -8.00 \\
\hline 34.50 & 37.64 & 30.00 & 27 & 24.00 \\
\hline-17.25 & -18.82 & -15.00 & -13.5 & -12.00 \\
\hline 28.75 & 31.36 & 25.00 & 22.5 & 20.00 \\
\hline-11.50 & -12.55 & -10.00 & -9 & -8.00 \\
\hline 63.25 & 69.00 & 55.00 & 49.5 & 44.00 \\
\hline 28.75 & 31.36 & 25.00 & 22.5 & 20.00 \\
\hline 126.50 & 138.00 & 110.00 & 99 & 88.00 \\
\hline-34.50 & -37.64 & -30.00 & -27 & -24.00 \\
\hline 120.75 & 131.73 & 105.00 & 94.5 & 84.00 \\
\hline 97.75 & 106.64 & 85.00 & 76.5 & 68.00 \\
\hline 103.50 & 112.91 & 90.00 & 81 & 72.00 \\
\hline-11.50 & -12.55 & -10.00 & -9 & -8.00 \\
\hline 63.25 & 69.00 & 55.00 & 49.5 & 44.00 \\
\hline-11.50 & -12.55 & -10.00 & -9 & -8.00 \\
\hline 97.75 & 106.64 & 85.00 & 76.5 & 68.00 \\
\hline-11.50 & -12.55 & -10.00 & -9 & -8.00 \\
\hline 63.25 & 69.00 & 55.00 & 49.5 & 44.00 \\
\hline-28.75 & -31.36 & -25.00 & -22.5 & -20.00 \\
\hline 195.50 & 213.27 & 170.00 & 153 & 136.00 \\
\hline-23.00 & -25.09 & -20.00 & -18 & -16.00 \\
\hline 5.75 & 6.27 & 5.00 & 4.5 & 4.00 \\
\hline-5.75 & -6.27 & -5.00 & -4.5 & -4.00 \\
\hline 23.00 & 25.09 & 20.00 & 18 & 16.00 \\
\hline
\end{tabular}




\begin{tabular}{|c|c|c|c|c|}
\hline-5.75 & -6.27 & -5.00 & -4.5 & -4.00 \\
\hline 40.25 & 43.91 & 35.00 & 31.5 & 28.00 \\
\hline-23.00 & -25.09 & -20.00 & -18 & -16.00 \\
\hline 28.75 & 31.36 & 25.00 & 22.5 & 20.00 \\
\hline-5.75 & -6.27 & -5.00 & -4.5 & -4.00 \\
\hline 23.00 & 25.09 & 20.00 & 18 & 16.00 \\
\hline 17.25 & 18.82 & 15.00 & 13.5 & 12.00 \\
\hline 34.50 & 37.64 & 30.00 & 27 & 24.00 \\
\hline-5.75 & -6.27 & -5.00 & -4.5 & -4.00 \\
\hline 23.00 & 25.09 & 20.00 & 18 & 16.00 \\
\hline 17.25 & 18.82 & 15.00 & 13.5 & 12.00 \\
\hline 23.00 & 25.09 & 20.00 & 18 & 16.00 \\
\hline-5.75 & -6.27 & -5.00 & -4.5 & -4.00 \\
\hline 23.00 & 25.09 & 20.00 & 18 & 16.00 \\
\hline 17.25 & 18.82 & 15.00 & 13.5 & 12.00 \\
\hline 57.50 & 62.73 & 50.00 & 45 & 40.00 \\
\hline 11.50 & 12.55 & 10.00 & 9 & 8.00 \\
\hline 28.75 & 31.36 & 25.00 & 22.5 & 20.00 \\
\hline-5.75 & -6.27 & -5.00 & -4.5 & -4.00 \\
\hline 86.25 & 94.09 & 75.00 & 67.5 & 60.00 \\
\hline 28.75 & 31.36 & 25.00 & 22.5 & 20.00 \\
\hline 126.50 & 138.00 & 110.00 & 99 & 88.00 \\
\hline-40.25 & -43.91 & -35.00 & -31.5 & -28.00 \\
\hline 161.00 & 175.64 & 140.00 & 126 & 112.00 \\
\hline 132.25 & 144.27 & 115.00 & 103.5 & 92.00 \\
\hline 138.00 & 150.55 & 120.00 & 108 & 96.00 \\
\hline-11.50 & -12.55 & -10.00 & -9 & -8.00 \\
\hline-5.75 & -6.27 & -5.00 & -4.5 & -4.00 \\
\hline-11.50 & -12.55 & -10.00 & -9 & -8.00 \\
\hline 120.75 & 131.73 & 105.00 & 94.5 & 84.00 \\
\hline 40.25 & 43.91 & 35.00 & 31.5 & 28.00 \\
\hline 149.50 & 163.09 & 130.00 & 117 & 104.00 \\
\hline 143.75 & 156.82 & 125.00 & 112.5 & 100.00 \\
\hline 189.75 & 207.00 & 165.00 & 148.5 & 132.00 \\
\hline-57.50 & -62.73 & -50.00 & -45 & -40.00 \\
\hline 230.00 & 250.91 & 200.00 & 180 & 160.00 \\
\hline 189.75 & 207.00 & 165.00 & 148.5 & 132.00 \\
\hline 212.75 & 232.09 & 185.00 & 166.5 & 148.00 \\
\hline-11.50 & -12.55 & -10.00 & -9 & -8.00 \\
\hline 17.25 & 18.82 & 15.00 & 13.5 & 12.00 \\
\hline-5.75 & -6.27 & -5.00 & -4.5 & -4.00 \\
\hline 17.25 & 18.82 & 15.00 & 13.5 & 12.00 \\
\hline-5.75 & -6.27 & -5.00 & -4.5 & -4.00 \\
\hline 80.50 & 87.82 & 70.00 & 63 & 56.00 \\
\hline-5.75 & -6.27 & -5.00 & -4.5 & -4.00 \\
\hline 115.00 & 125.45 & 100.00 & 90 & 80.00 \\
\hline-11.50 & -12.55 & -10.00 & -9 & -8.00 \\
\hline 40.25 & 43.91 & 35.00 & 31.5 & 28.00 \\
\hline
\end{tabular}




\begin{tabular}{|c|c|c|c|c|}
\hline-11.50 & -12.55 & -10.00 & -9 & -8.00 \\
\hline 28.75 & 31.36 & 25.00 & 22.5 & 20.00 \\
\hline-11.50 & -12.55 & -10.00 & -9 & -8.00 \\
\hline 74.75 & 81.55 & 65.00 & 58.5 & 52.00 \\
\hline 17.25 & 18.82 & 15.00 & 13.5 & 12.00 \\
\hline 97.75 & 106.64 & 85.00 & 76.5 & 68.00 \\
\hline-34.50 & -37.64 & -30.00 & -27 & -24.00 \\
\hline 126.50 & 138.00 & 110.00 & 99 & 88.00 \\
\hline-17.25 & -18.82 & -15.00 & -13.5 & -12.00 \\
\hline-5.75 & -6.27 & -5.00 & -4.5 & -4.00 \\
\hline-11.50 & -12.55 & -10.00 & -9 & -8.00 \\
\hline 34.50 & 37.64 & 30.00 & 27 & 24.00 \\
\hline 17.25 & 18.82 & 15.00 & 13.5 & 12.00 \\
\hline 40.25 & 43.91 & 35.00 & 31.5 & 28.00 \\
\hline-23.00 & -25.09 & -20.00 & -18 & -16.00 \\
\hline 69.00 & 75.27 & 60.00 & 54 & 48.00 \\
\hline
\end{tabular}




\section{Appendix B \\ Programming Code}

\begin{tabular}{|c|c|c|}
\hline $\begin{array}{l}\text { Symbols } \\
\text { (in Code) }\end{array}$ & $\begin{array}{l}\text { Symbols } \\
\text { (in Text) }\end{array}$ & Variable Description \\
\hline e & E. & Modulus of elasticity \\
\hline $\mathrm{k}$ & $\mathrm{K}^{\prime}$ & Cyclic Strength Coefficient \\
\hline $\mathrm{n}$ & $1 / n^{\prime}$ & Cyclic Strain Hardening Exponent \\
\hline $\mathrm{Ve}$ & $v_{e}$ & Elastic Poisson's ratio \\
\hline $\mathrm{Vp}$ & $v_{p}$ & Plastic Poisson's ratio \\
\hline Veff & $v_{e f f}$ & Effective Poisson's ratio \\
\hline EA & $\varepsilon_{t}$ & Total Strain \\
\hline EP & $\varepsilon_{p}$ & Plastic Strain \\
\hline $\mathrm{EE}$ & $\varepsilon_{e}$ & Elastic Strain \\
\hline $\mathrm{Ya}$ & $\left(\frac{\gamma_{a}}{2}\right)$ & Shear Strain \\
\hline $\mathrm{Ta}$ & $\tau_{a}$ & Shear Stress \\
\hline SF & $\sigma_{f}$ & Fatigue Axial Strength Coefficient \\
\hline EF & $\varepsilon_{f}^{\prime}$ & Fatigue Axial Ductility Coefficient \\
\hline ST & $\tau_{f}^{\prime}$ & Shear Fatigue Strength Coefficient \\
\hline ET & $\gamma_{f}$ & Shear Fatigue Ductility Coefficient \\
\hline $\mathrm{G}$ & G & Shear Modulus \\
\hline $\mathrm{b}$ & b & Fatigue Strength Exponent \\
\hline c & c & Fatigue Ductility Exponent \\
\hline b1 & $b^{\prime}$ & Fatigue Torsional Strength Exponent \\
\hline c1 & $c^{\prime}$ & Fatigue Torsional Ductility Exponent \\
\hline SR & $\Delta \sigma_{n}$ & Normal Axial Stress Range \\
\hline ER & $\Delta \varepsilon_{n}$ & Normal Axial Strain Range \\
\hline STR & $\Delta \tau_{\max }$ & Maximum Shear Stress Range \\
\hline ETR & $\Delta\left(\frac{\gamma_{\max }}{2}\right)$ & Maximum Shear Strain Range \\
\hline
\end{tabular}




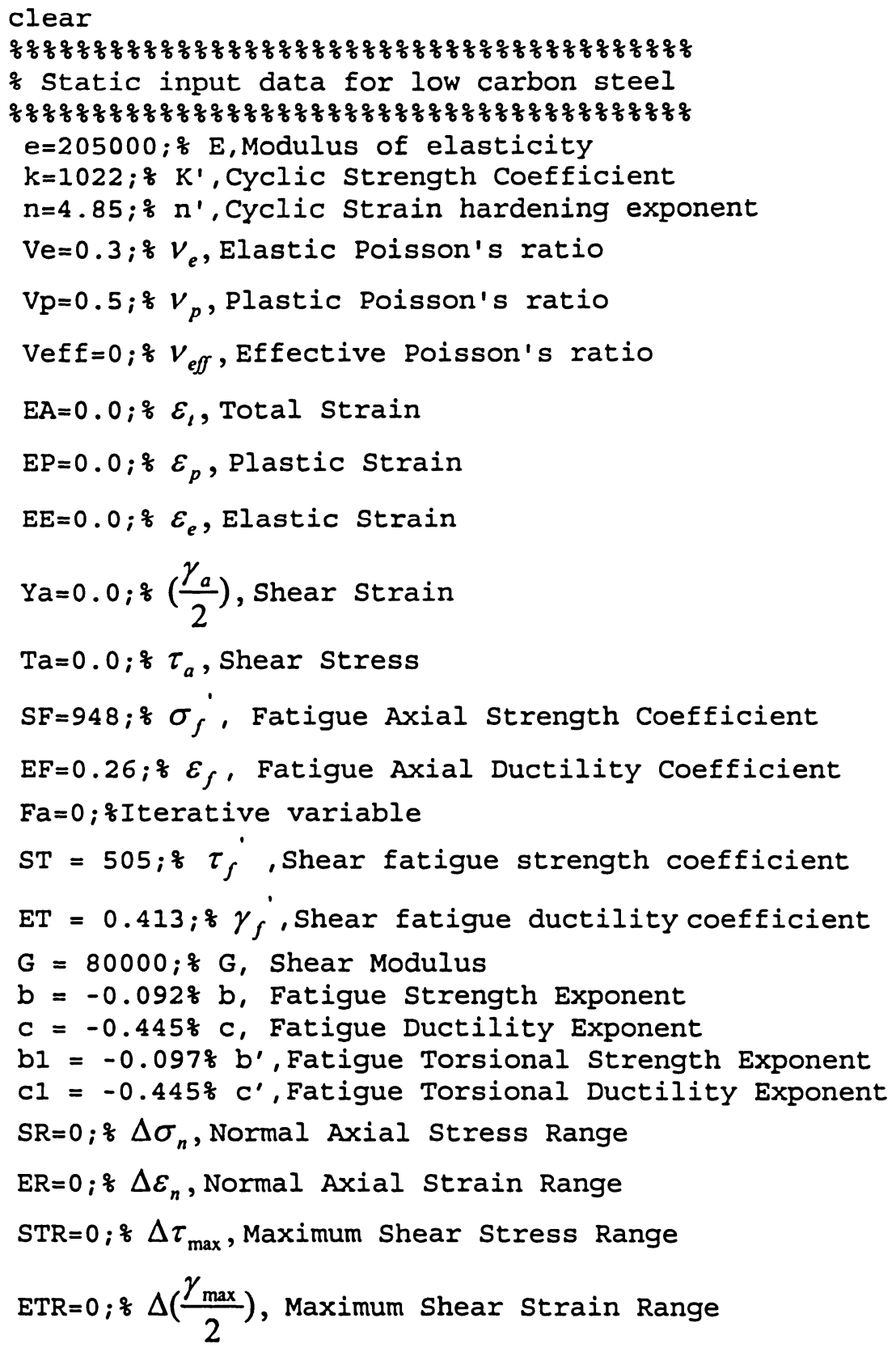


$j=p / e+(p / k) \cdot{ }^{\wedge} n \& \varepsilon_{a}, j$ represents strain values with respect to $p$.

$E 1=$ length $(j)$ \& $E 1$ tells the length of $j$

$E A=j(E 1) ;$ \& $E A$ traps the last value of $E 1$

$\mathrm{EE}=\mathrm{EA} / \mathrm{e}$ \& Calculate the Elastic strain

$E P=E A-E E ;$ \& Calculate the plastic strain from total strain

Veff $=(V e \star E E+V p \star E P) /(E E+E P)$ \& Calculate the effective Poisson's ratio.

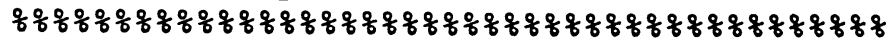

\% E11,E12,E13 calculate Principal strains

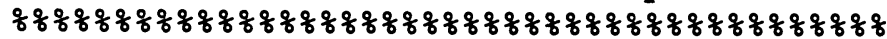

$E 11=(1-V e f f) \star E A / 2+0.5 *\left(\left(E A^{\wedge} 2\right) *(1+\operatorname{Veff})+(\mathrm{Ya} / 2) \wedge 2\right)^{\wedge} 0.5$

$E 12=-\operatorname{Vef} * \mathrm{EA}$

$E 13=(1-$ Veff $) \star E A / 2-0.5 *\left(\left(E A^{\wedge} 2\right) *(1+\operatorname{Veff})+(\text { Ya } / 2)^{\wedge} 2\right)^{\wedge} 0.5$

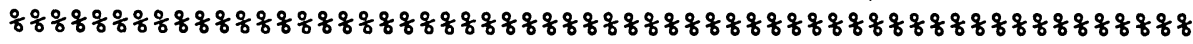

sLogic to get maximum and minimum principal strain values

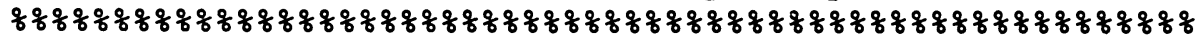

if $(E 12<E 13 \& E 11>E 13)$

$\mathrm{E} 13=\mathrm{E} 12$

$E 11=E 11$

elseif $(E 12>E 13 \& E 11<E 13)$

$\mathrm{E} 11=\mathrm{E} 12$

$\mathrm{E} 13=\mathrm{E} 13$

elseif $(E 12<E 11 \& E 13>E 11)$

$\mathrm{E} 11=\mathrm{E} 13$

$\mathrm{E} 13=\mathrm{E} 12$

else

$\mathrm{E} 13=\mathrm{E} 13$

$\mathrm{E} I 1=\mathrm{E} I 1$

end

$j 1=$ length $(p) ;$ o $j l$ tells the length of $p$

$J=p(j 1)$; $J$ traps the last value of $j I$

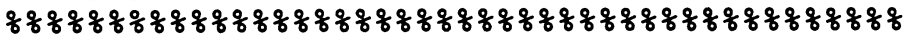

\% S11,S12,S13 calculate Principal stresses

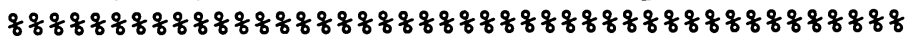

$\mathrm{S} 11=\mathrm{J} / 2+0.5 *\left(\mathrm{~J}^{\wedge} 2+4{ }^{\star} \mathrm{Ta}^{\wedge} 2\right) \wedge 0.5$

$\mathrm{S} 12=0$

$S 13=J / 2-0.5 *\left(J^{\wedge} 2+4 *^{\top} \mathrm{Ta}^{\wedge} 2\right) \wedge 0.5$

$\mathrm{P}=$ length $(A)$ \& $P$ tells the length of $A$

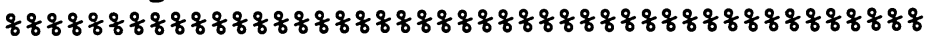

isecond Stress input is taken from the file

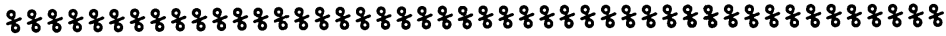

$\mathrm{y}=\mathrm{A}(2) \quad$ of Inputting second stress value

$y 1=a b s(y * 0.10)$ Dividing Input stress into equal intervals

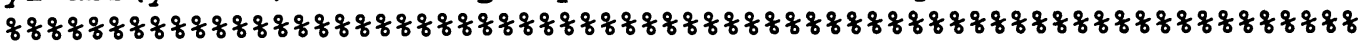

$\% \mathrm{~J}$ compares with max. value and $y$ with minimum to trap max. value

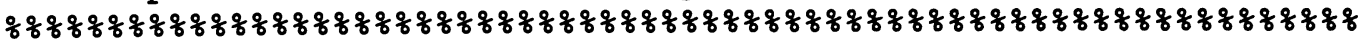
if $(J==A A \& Y \sim=B B \&$ cont $1==1$ )

$\mathrm{q}=\mathrm{J}: \mathrm{Y} / \mathrm{Y} 1: \mathrm{Y} ; \% \sigma_{a}, \mathrm{q}$ represent stress values from $\mathrm{J}$ to $\mathrm{Y}$

$\mathrm{h}=\mathrm{EA}-((\mathrm{J}-(\mathrm{q})) / \mathrm{e})-2 \star((\mathrm{J}-(\mathrm{q})) /(2 \star \mathrm{k})) .^{\wedge} \mathrm{h} q \mathrm{~h}$ is $\varepsilon_{a}$, strain values 


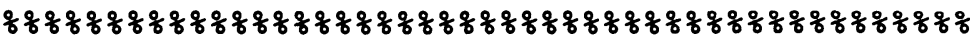

o BB1 Record the min. range from the last data

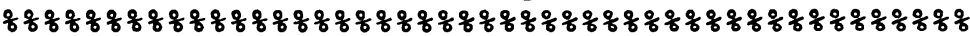

$\mathrm{BBI}=\mathrm{abs}(0.1 * \mathrm{BB})$

$\mathrm{y} l \mathrm{r}=\mathrm{J}: \mathrm{BB} / \mathrm{BB} 1: \mathrm{BB} ; \quad \frac{\sigma}{a}, \mathrm{y} 1 \mathrm{r}$ represent stress values from $\mathrm{J}$ to $\mathrm{BB}$ $\mathrm{y} I \mathrm{i}=\mathrm{EA}-((\mathrm{J}-(\mathrm{y} l \mathrm{r})) / \mathrm{e})-2 *((J-(y \perp r)) /(2 \star \mathrm{k})) \cdot{ }^{\wedge} \mathrm{n}$

\& $y 1 i$ is $\varepsilon_{a}$, strain values

$\mathrm{hl}=$ length $(\mathrm{q})$

$\mathrm{H}=\mathrm{q}(\mathrm{hl})$;

$E 2$ = length $(h)$

$E B=h(E 2) ;$

$E E=E B / e$ o Calculate the Elastic strain

$E P=E B-E E \&$ Calculate the plastic strain from total strain

Veff $=\left(V e^{\star} E E+V p \star E P\right) /(E E+E P) \&$ Calculate the effective Poisson's ratio

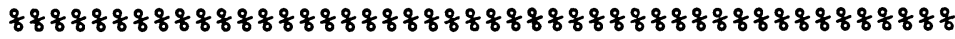

\% E111,E112,E113 calculate Principal strains

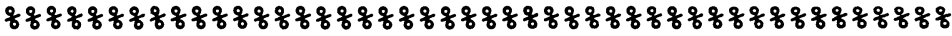

$E I 11=(1-\operatorname{Veff}) * E B / 2+0.5 *\left((E B \wedge 2) *(1+\operatorname{Veff})+(\mathrm{Ya} / 2)^{\wedge} 2\right) \wedge 0.5$

$E 112=-$ Vef $f \star E B$

$E 113=(1-$ Veff $) \star E B / 2-0.5 *\left(\left(E B^{\wedge} 2\right) *(1+\operatorname{Veff})+(\text { Ya } / 2)^{\wedge} 2\right)^{\wedge} 0.5$

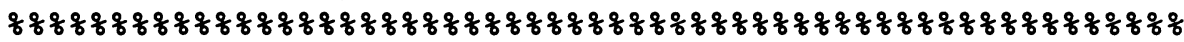

Logic to get maximum and minimum principal strain values

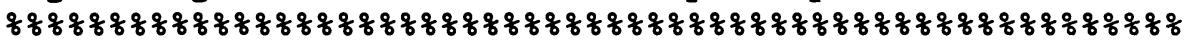

if $(E 112<E 113 \& E 111>E 113)$

$\mathrm{E} 113=\mathrm{E} 112$

$E 111=E 111$

elseif (E112 > E113\&E111 < E113)

$E 111=E 112$

$E 113=E 113$

elseif (E112 < E111 \& E113>E111)

$E 111=E 113$

$E 113=E 112$

else

$\mathrm{E} 113=\mathrm{E} 113$

$E 111=E 111$

End

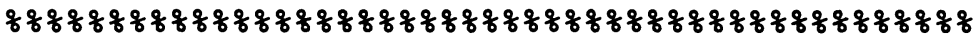

\% S111,S112,S113 calculate Principal stresses

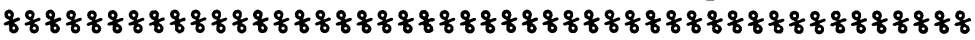

$S 111=\mathrm{H} / 2+0.5 *\left(\mathrm{H}^{\wedge} 2+4{ }^{\mathrm{T}} \mathrm{Ta} \wedge 2\right)^{\wedge} 0.5$

$\mathrm{S} 112=0$

$\mathrm{S} 113=\mathrm{H} / 2-0.5 *\left(\mathrm{H}^{\wedge} 2+4{ }^{\mathrm{T}} \mathrm{Ta} \mathrm{A}^{\wedge} 2\right) \wedge .5$

else

cont $1=\operatorname{cont} I+1$

$\mathrm{q}=\mathrm{J}: \mathrm{y} / \mathrm{Y} 1: \mathrm{y} ; \quad$ $\sigma_{a}, \mathrm{q}$ represent stress values from $\mathrm{J}$ to $\mathrm{y}$

$\mathrm{h}=\mathrm{EA}-((\mathrm{J}-(\mathrm{q})) / \mathrm{e})-2 \star((\mathrm{J}-(\mathrm{q})) /(2 \star \mathrm{k})) \cdot{ }^{\wedge} \mathrm{n} \& \mathrm{~h}$ is $\varepsilon_{a}$, strain

$\mathrm{hl}=$ length $(\mathrm{q})$

$\mathrm{H}=\mathrm{q}(\mathrm{hl})$;

$E 2$ = length $(\mathrm{h})$

$E B=h(E 2) ;$

$\mathrm{EE}=\mathrm{EB} / \mathrm{e}$ \& Calculate the Elastic strain

$E P=E B-E E$ Calculate the plastic strain from total strain

Veff $=(V e \star E E+V p \star E P) /(E E+E P)$ \& Calculate the effective Poisson's ratio 


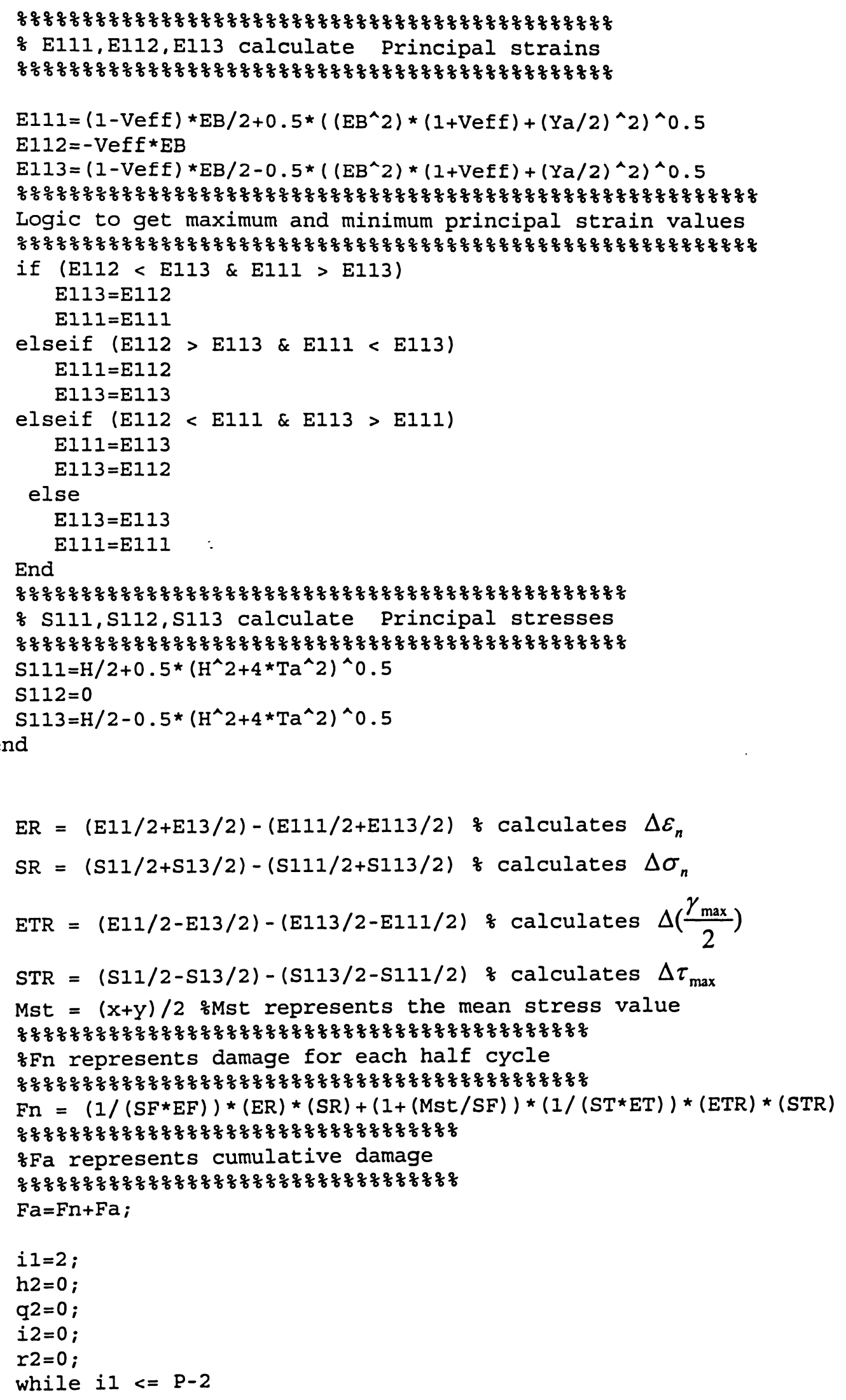




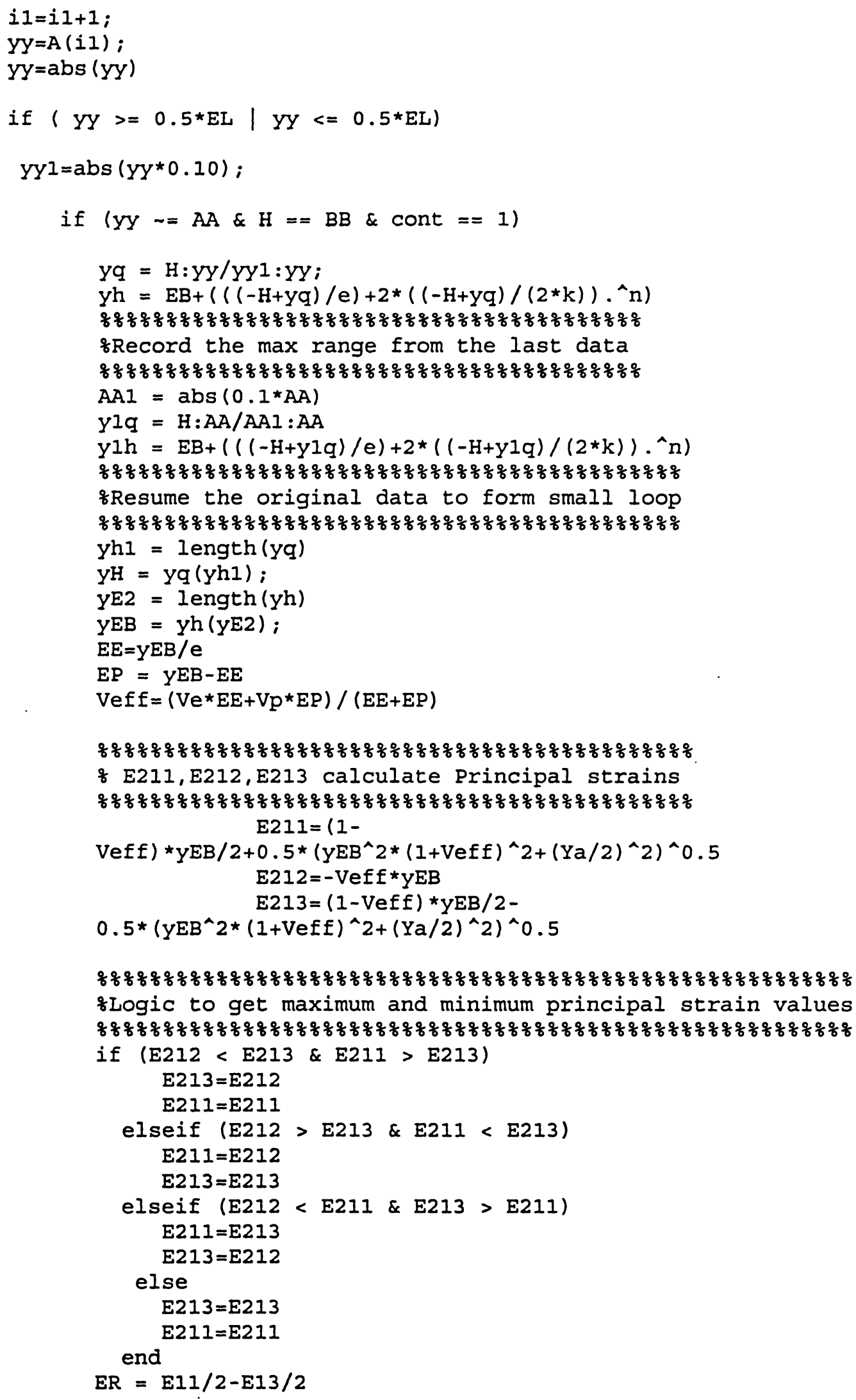




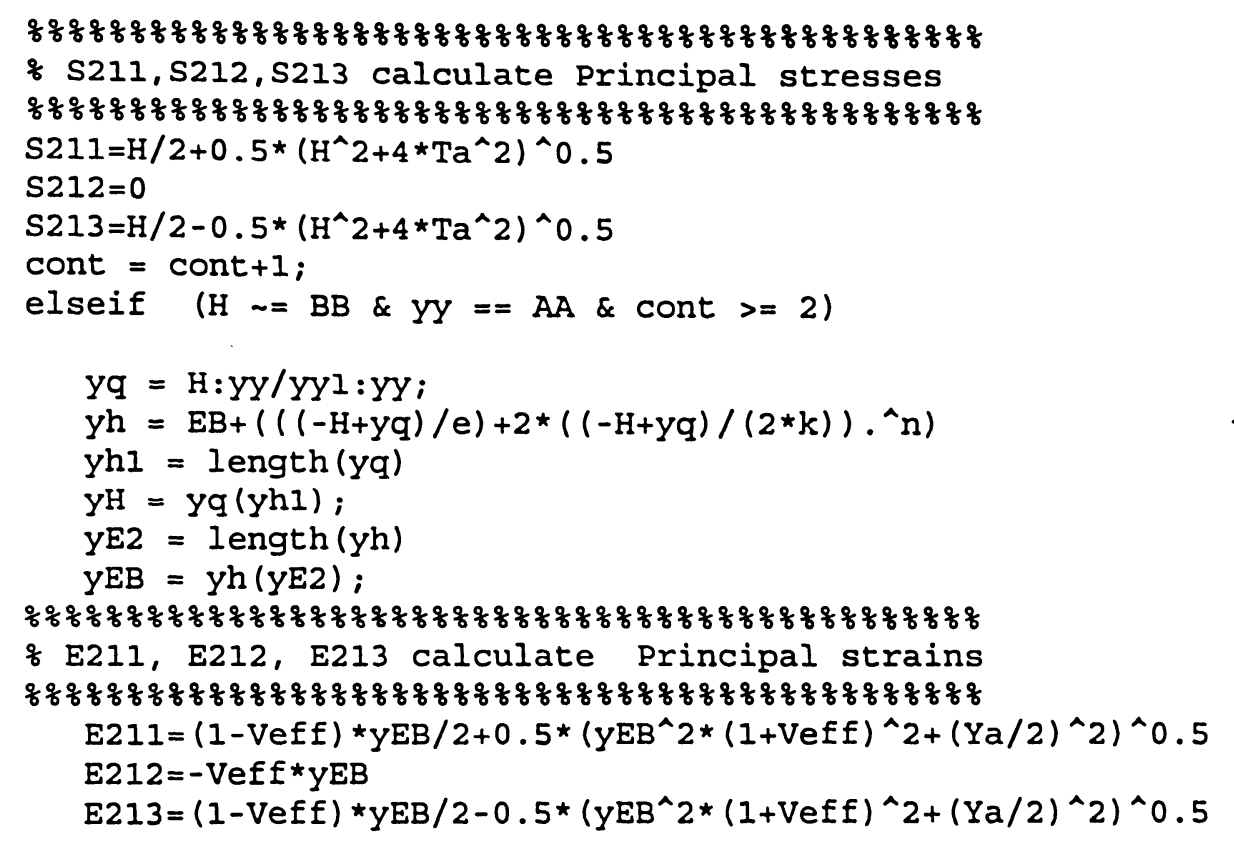

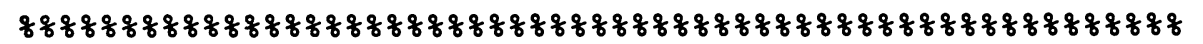
flogic to get maximum and minimum principal strain values

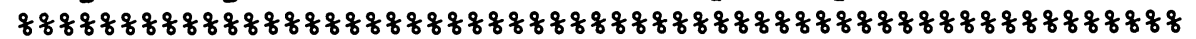




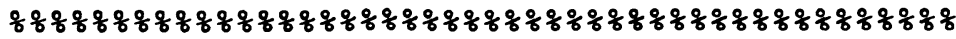

\% E211, E212, E213 calculate Principal strains

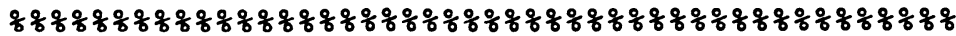

$$
\begin{aligned}
& E 211=(1-\operatorname{Veff}) \star y E B / 2+0.5 *\left(y E B \wedge 2 *(1+\operatorname{Veff})^{\wedge} 2+(Y a / 2) \wedge 2\right)^{\wedge} 0.5 \\
& E 212=- \text { Veff } * Y E B \\
& \mathrm{E} 213=(1-\operatorname{Veff}) * \mathrm{YEB} / 2-0.5 *\left(\mathrm{YEB} \mathrm{B}^{\wedge} 2 *(1+\operatorname{Veff})^{\wedge} 2+(\mathrm{Ya} / 2)^{\wedge} 2\right)^{\wedge} 0.5
\end{aligned}
$$

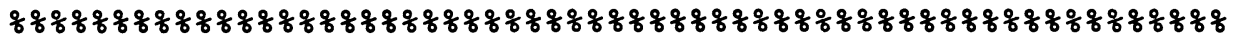
qLogic to get maximum and minimum principal strain values

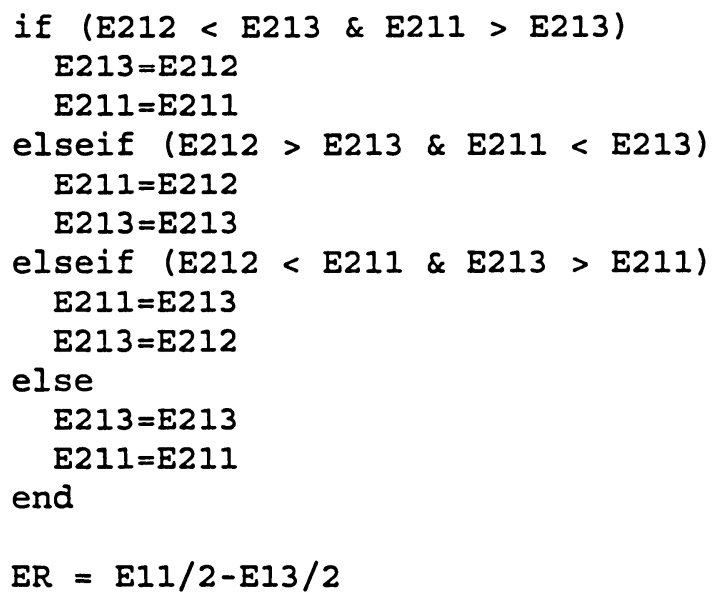

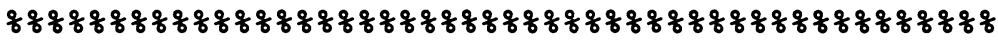
\% S211, S212, S213 calculate Principal stresses

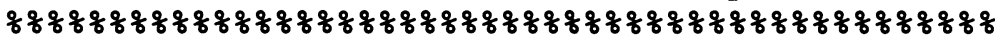

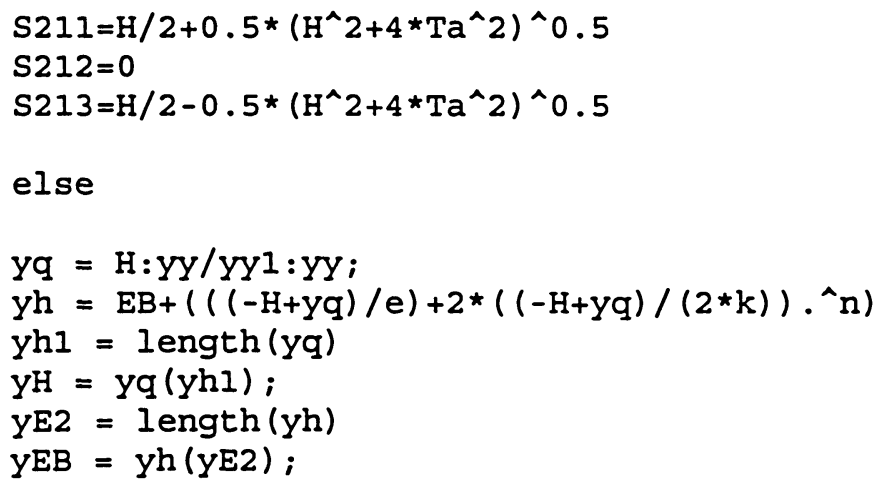

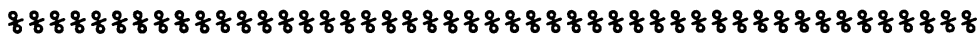
\% E211, E212, E213 calculate Principal strains

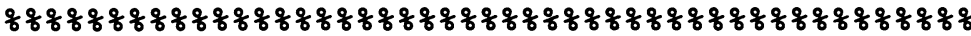

$\mathrm{E} 211=(1-\mathrm{Veff}) * \mathrm{YEB} / 2+0.5 *\left(\mathrm{YEB}^{\wedge} 2 *(1+\mathrm{Veff})^{\wedge} 2+(\mathrm{Ya} / 2)^{\wedge} 2\right)^{\wedge} 0.5$ E212 $=-$ Veff*yEB

$\mathrm{E} 213=(1-\operatorname{Veff}) \star \mathrm{YEB} / 2-0.5 *\left(\mathrm{YEB}^{\wedge} 2 *(1+\operatorname{Veff})^{\wedge} 2+(\mathrm{Ya} / 2)^{\wedge} 2\right)^{\wedge} 0.5$

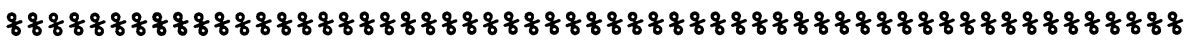
flogic to get maximum and minimum principal strain values

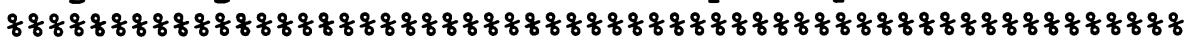
if $(E 212<E 213 \& E 211>E 213)$

$\mathrm{E} 213=\mathrm{E} 212$

$E 211=E 211$ 


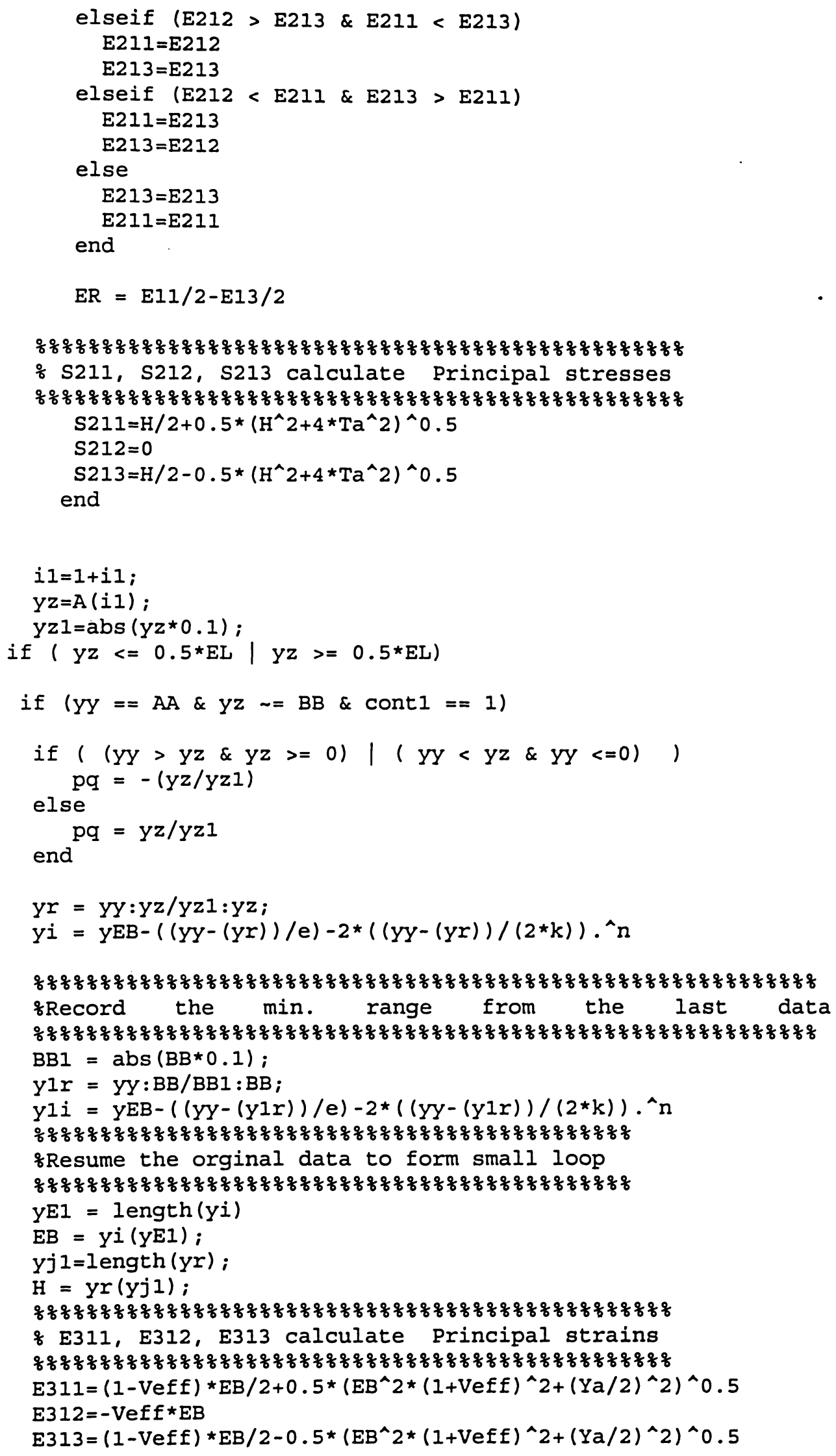




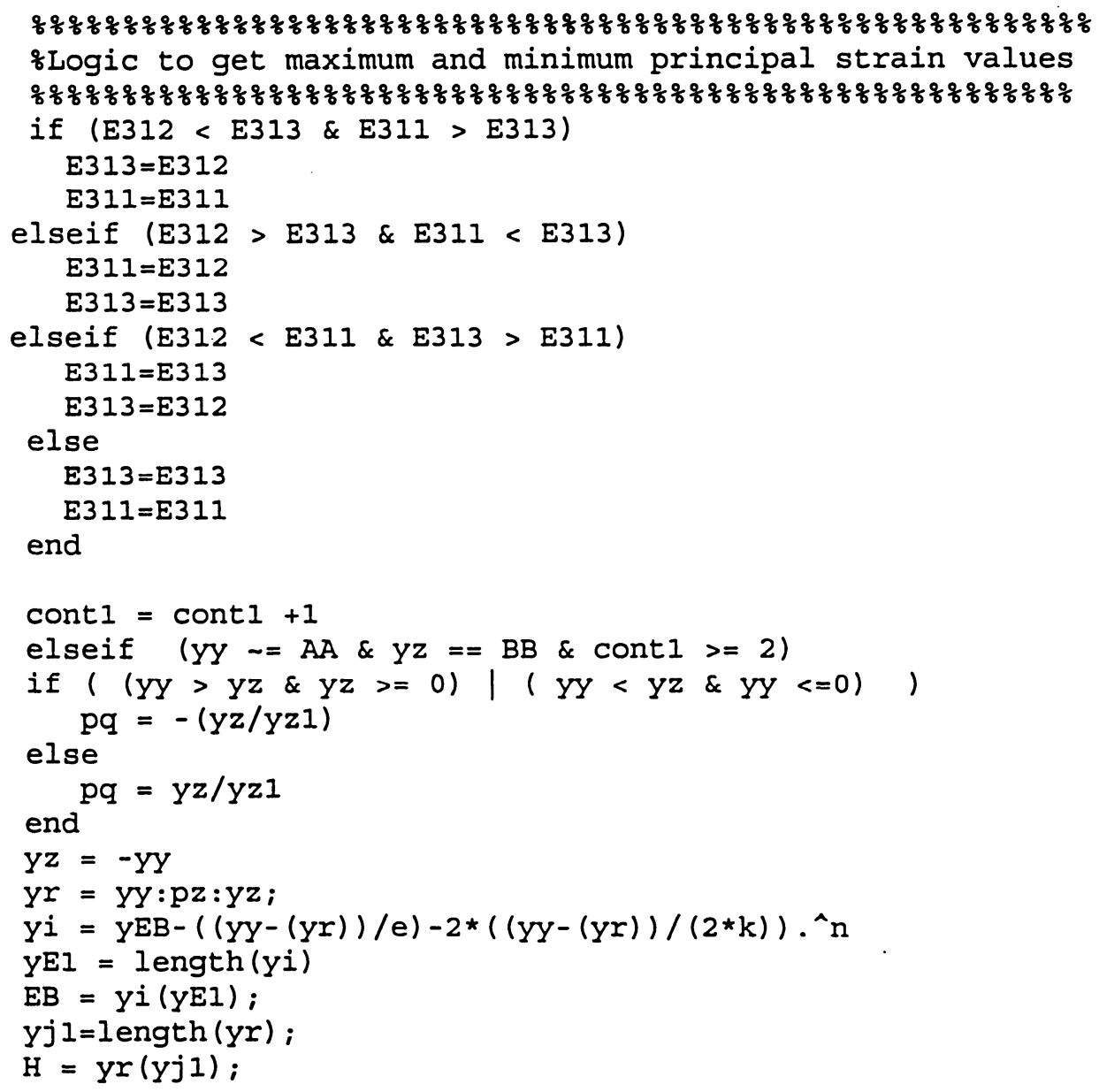




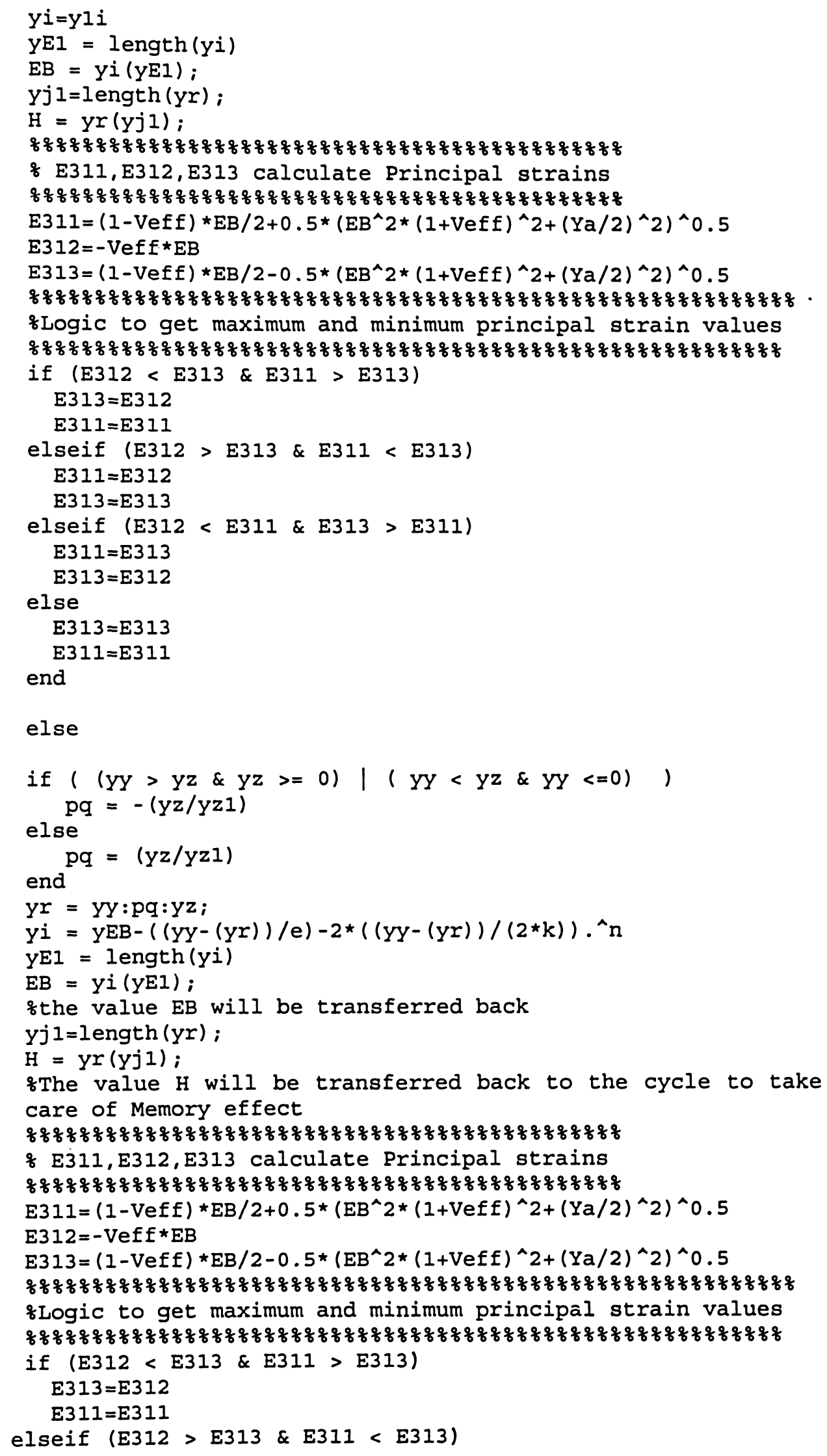




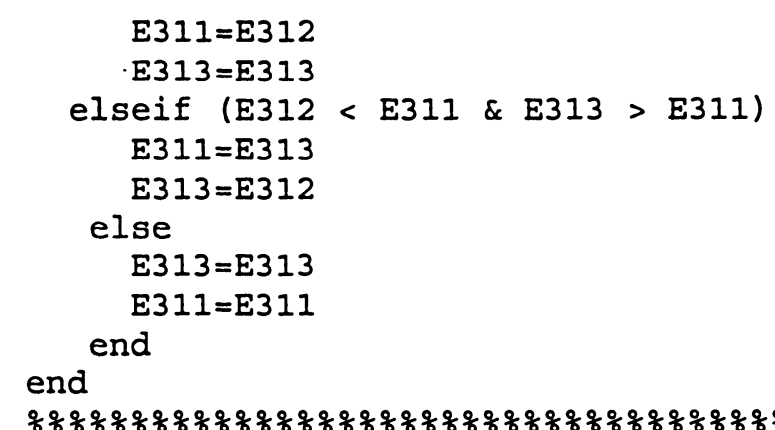

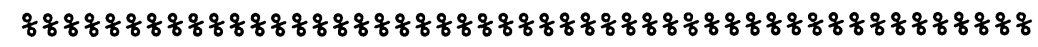

\% S211, S212, S213 calculate Principal stresses

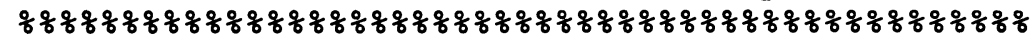
$\mathrm{S} 311=\mathrm{H} / 2+0.5 *\left(\mathrm{H}^{\wedge} 2+4{ }^{\top} \mathrm{Ta} \mathrm{A}^{\wedge} 2\right) \wedge 0.5$ 


$$
\text { Ek. o d ic }
$$

